\title{
The Discreet Structural Diversity of Briarellins: DU8+ Guided Multiple Structure Revisions Yielded Two Unknown Structural Types.
}

Tina A. Holt, ${ }^{a}$ D. Sai Reddy, ${ }^{a}$ Deepak B. Huple, ${ }^{a}$ Lyndon M. West, ${ }^{d}$ Abimael D. Rodriquez, ${ }^{c}$ Michael T. Crimmins, ${ }^{b}$ and Andrei G. Kutateladze ${ }^{\star a}$

†Department of Chemistry and Biochemistry, University of Denver, Denver, CO 80208

\#Department of Chemistry and Biochemistry, Florida Atlantic University, Boca Raton, FL 33431

\&Molecular Science Research Center, University of Puerto Rico, 1390 Ponce de León Avenue, San Juan, oog26, Puerto Rico

‡Department of Chemistry, University of North Carolina, Chapel Hill, NC, 27599-3290

\section{SUPPORTING INFORMATION}

\section{Table of Contents}

\begin{tabular}{|c|c|}
\hline 3riarellin A . & $-\quad$ revised ................................ \\
\hline riarellin B & - original.............. \\
\hline riarellin B & - revised................. \\
\hline fiarellin D & - original... \\
\hline riarellin D & - revised ............................. \\
\hline riarellin $\mathrm{E}$ & - original (correct).. \\
\hline riarellin $\mathrm{F}$ & - original (correct). \\
\hline riarellin $\mathrm{H}$ & - original......................... \\
\hline riarellin $\mathrm{H}$ & - revised ............................... \\
\hline riarellin $\mathrm{H}$ & 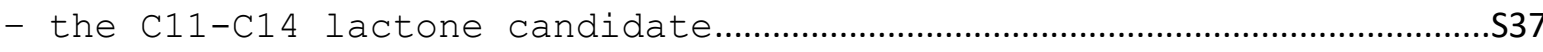 \\
\hline riarellin J & 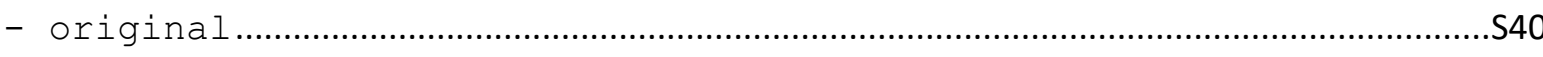 \\
\hline iarellin J & - revised........ \\
\hline riarellins $\mathrm{K}$ & - original... \\
\hline arellin $\mathrm{K}$ & 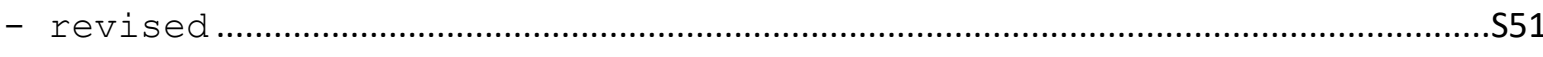 \\
\hline iarellin $\mathrm{L}$ & - original ..................................... \\
\hline arellin L & - revised.................. \\
\hline arellins M & sed \\
\hline estinin-21 & 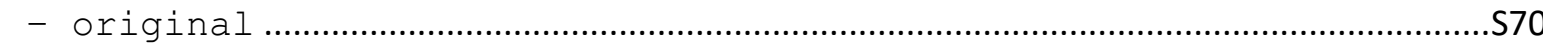 \\
\hline estinin-21 & - first revision (the 7-epimer of the final revision below) ...........S75 \\
\hline-21 & 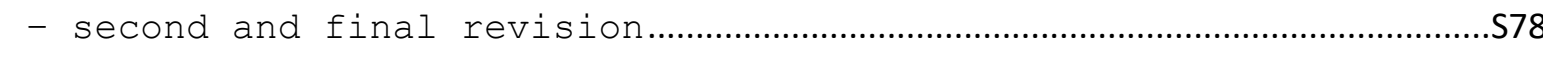 \\
\hline
\end{tabular}


Notes:

(a) in all calculations the acyls are truncated to acetyls

(b) the chemical shifts reassignments are denoted by asterisks

Briarellin A - original

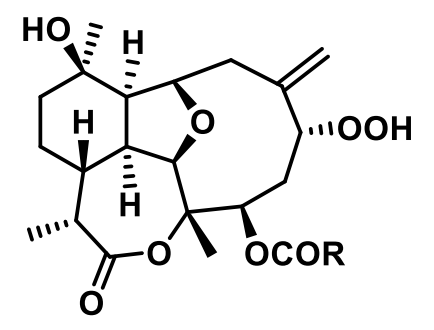

(the best fit to the experimental data still gives unacceptable rmsd > 4ppm)

Rel energy (kcal/mol): $\begin{array}{cccc}\text { Conf } 1 & \text { Conf } 2 & \text { Conf } 3 & \text { Conf } 4 \\ 0.1 & 0.9 & 0.0 & 1.3\end{array}$

\begin{tabular}{|c|c|c|c|c|c|c|c|c|c|}
\hline C-nom & iGau & $\operatorname{Exp}$ & Calc & $\operatorname{diff}$ & 1 & 2 & 3 & 4 & \\
\hline$C-C$ & 8 & 176.00 & 181.93 & 5.93 & [ 181.97 & 182.05 & 181.85 & 182.02 & ] \\
\hline $\mathrm{C}-\mathrm{C}$ & 19 & 142.30 & 145.65 & 3.35 & {$[146.59$} & 142.81 & 145.46 & 145.66 & ] \\
\hline $\mathrm{C}-\mathrm{C}$ & 6 & 80.50 & 87.40 & 6.90 & {$\left[\begin{array}{l}87.61 \\
0\end{array}\right.$} & 87.94 & 87.08 & 87.41 & ] \\
\hline$C-C$ & 13 & 74.30 & 71.95 & -2.35 & 72.11 & 72.13 & 71.80 & 71.60 & ] \\
\hline $\mathrm{C}-\mathrm{CH}$ & 5 & 92.50 & 94.00 & 1.50 & 93.39 & 93.56 & 94.64 & 94.05 & ] \\
\hline $\mathrm{C}-\mathrm{CH}$ & 18 & 82.90 & 88.74 & 5.84 & 90.94 & 85.66 & 87.46 & 89.19 & ] \\
\hline $\mathrm{C}-\mathrm{CH}$ & 15 & 82.40 & 82.38 & -0.02 & 82.33 & 82.40 & 82.37 & 82.83 & ] \\
\hline $\mathrm{C}-\mathrm{CH}$ & 10 & 72.20 & 80.09 & 7.89 & 79.23 & 81.61 & 80.67 & 78.42 & ] \\
\hline $\mathrm{C}-\mathrm{CH}$ & 14 & 47.60 & 51.30 & 3.70 & 49.94 & 49.06 & 52.88 & 52.67 & ] \\
\hline $\mathrm{C}-\mathrm{CH}$ & 2 & 45.20 & 47.52 & 2.32 & 47.44 & 47.53 & 47.60 & 47.39 & ] \\
\hline $\mathrm{C}-\mathrm{CH}$ & 4 & 44.70 & 41.84 & -2.86 & 42.87 & 42.51 & 40.88 & 40.77 & ] \\
\hline $\mathrm{C}-\mathrm{CH}$ & 3 & 37.50 & 38.77 & 1.27 & [ 38.93 & 39.26 & 38.55 & 38.52 & ] \\
\hline $\mathrm{C}-\mathrm{CH} 2$ & 42 & 119.70 & 116.17 & -3.53 & [ 113.28 & 116.92 & 118.07 & 120.73 & ] \\
\hline $\mathrm{C}-\mathrm{CH} 2$ & 43 & 42.20 & 40.06 & -2.14 & {$[40.43$} & 40.42 & 39.76 & 39.07 & ] \\
\hline $\mathrm{C}-\mathrm{CH} 2$ & 12 & 34.80 & 38.12 & 3.32 & 37.69 & 37.86 & 38.49 & 38.74 & ] \\
\hline $\mathrm{C}-\mathrm{CH} 2$ & 17 & 30.30 & 32.08 & 1.78 & 30.54 & 27.50 & 34.33 & 33.67 & ] \\
\hline $\mathrm{C}-\mathrm{CH} 2$ & 9 & 16.30 & 23.68 & 7.38 & 23.75 & 23.76 & 23.61 & 23.58 & ] \\
\hline $\mathrm{C}-\mathrm{CH} 3$ & 34 & 28.80 & 28.66 & -0.14 & 28.75 & 28.68 & 28.60 & 28.53 & \\
\hline $\mathrm{C}-\mathrm{CH} 3$ & 27 & 22.50 & 22.48 & -0.02 & 22.12 & 23.25 & 22.71 & 21.62 & ] \\
\hline \multirow[t]{3}{*}{$\mathrm{C}-\mathrm{CH} 3$} & 1 & 17.50 & 9.19 & -8.31 & {$[\quad 9.26$} & 9.29 & 9.11 & 9.11 & ] \\
\hline & & $13 \mathrm{C} \mathrm{cl}$ & & is: RMS & $4.37 \mathrm{ppm}$ & $(\mathrm{MAE}=3.5$ & 53) $\mathrm{N}=20$ & $\{-8.31$ & $7.89\}$ \\
\hline & & & & racti & $: 0.399$ & 0.103 & 0.451 & 0.047 & \\
\hline
\end{tabular}

Conformer 1

Energy: -1459.25481 Hartree (Rel: $0.1 \mathrm{kcal} / \mathrm{mol}$ )

XYZ coordinates for conf 1 :

$\begin{array}{lrrr}\text { C } & 0.98449 & -2.67053 & 2.45013 \\ \text { C } & 1.56674 & -2.75677 & 1.02957 \\ \text { C } & 2.34140 & -1.48457 & 0.54845 \\ \text { C } & 1.41352 & -0.28845 & 0.24610 \\ C & 0.60923 & -0.46271 & -1.05809 \\ C & -0.79690 & -1.12458 & -0.86679 \\ \text { O } & -0.58536 & -2.19964 & 0.10879 \\ C & 0.50252 & -3.03148 & -0.02343 \\ C & 3.43782 & -1.02714 & 1.51916 \\ C & -1.76387 & -0.15991 & -0.11763\end{array}$




\begin{tabular}{|c|c|c|}
\hline 0.59648 & -3.85787 & -0.90310 \\
\hline 4.33333 & 0.05644 & 0.87418 \\
\hline 3.65151 & 0.91188 & -0.23422 \\
\hline 2.14655 & 1.07282 & 0.06753 \\
\hline 1.34348 & 1.79399 & -1.04716 \\
\hline 0.49622 & 0.81554 & -1.66802 \\
\hline-2.24268 & 1.07097 & -0.91502 \\
\hline-2.03295 & 2.42225 & -0.19702 \\
\hline-0.64074 & 2.67918 & 0.38399 \\
\hline 1.78784 & -2.55670 & 3.18434 \\
\hline 0.43901 & -3.58800 & 2.69680 \\
\hline 0.29104 & -1.83298 & 2.56078 \\
\hline 2.26885 & -3.59659 & 0.98565 \\
\hline 2.83377 & -1.76571 & -0.38945 \\
\hline 0.72430 & -0.17185 & 1.08716 \\
\hline 1.17285 & -1.11488 & -1.74104 \\
\hline-1.31729 & -1.68642 & -2.18891 \\
\hline 2.97398 & -0.62706 & 2.42978 \\
\hline 4.05437 & -1.87759 & 1.83468 \\
\hline-2.83849 & -0.88041 & 0.55271 \\
\hline-1.20765 & 0.19158 & 0.75068 \\
\hline 5.21544 & -0.41026 & 0.41613 \\
\hline 4.71550 & 0.72032 & 1.65860 \\
\hline 4.37080 & 2.26517 & -0.34521 \\
\hline 3.71279 & 0.23421 & -1.50405 \\
\hline 2.07798 & 1.63893 & 1.00133 \\
\hline 2.02365 & 2.16194 & -1.82225 \\
\hline 4.26327 & 2.85153 & 0.57410 \\
\hline-3.30460 & 0.98541 & -1.14503 \\
\hline-1.71502 & 1.12446 & -1.86539 \\
\hline-3.05142 & 2.48297 & 0.81089 \\
\hline-0.44133 & 2.67280 & 1.70999 \\
\hline 0.47769 & 2.99619 & -0.59335 \\
\hline 0.52280 & 2.92251 & 2.14441 \\
\hline-1.23365 & 2.40552 & 2.40448 \\
\hline-3.97200 & -1.28418 & -0.05536 \\
\hline 0.05454 & 3.45077 & -1.49820 \\
\hline 1.15280 & 3.74150 & -0.15508 \\
\hline 3.98439 & 2.85471 & -1.18226 \\
\hline 5.44421 & 2.10859 & -0.51348 \\
\hline 4.64868 & 0.13682 & -1.74499 \\
\hline-4.88976 & -1.93912 & 0.95199 \\
\hline-4.22834 & -1.14458 & -1.23524 \\
\hline-4.39197 & -2.80226 & 1.40578 \\
\hline-5.80681 & -2.25889 & 0.45594 \\
\hline-5.12706 & -1.23571 & 1.75657 \\
\hline-2.26200 & -2.21229 & -2.05405 \\
\hline-0.58222 & -2.37927 & -2.60563 \\
\hline-1.47597 & -0.87669 & -2.90815 \\
\hline-2.24769 & 3.21450 & -0.92676 \\
\hline-3.26050 & 3.87566 & 1.16256 \\
\hline-2.51508 & 4.03566 & 1.77487 \\
\hline
\end{tabular}

Conformer 2

Energy: -1459.25354 Hartree (Rel: $0.9 \mathrm{kcal} / \mathrm{mol}$ ) XYZ coordinates for conf 2 :

$\begin{array}{rrrr}C & -0.86412 & 2.64505 & 2.44851 \\ C & -1.43358 & 2.76939 & 1.02584 \\ C & -2.28050 & 1.54697 & 0.53972 \\ C & -1.42511 & 0.29594 & 0.24645 \\ C & -0.60122 & 0.42606 & -1.04952 \\ C & 0.84318 & 0.99490 & -0.85034 \\ \text { O } & 0.69108 & 2.09468 & 0.11216\end{array}$




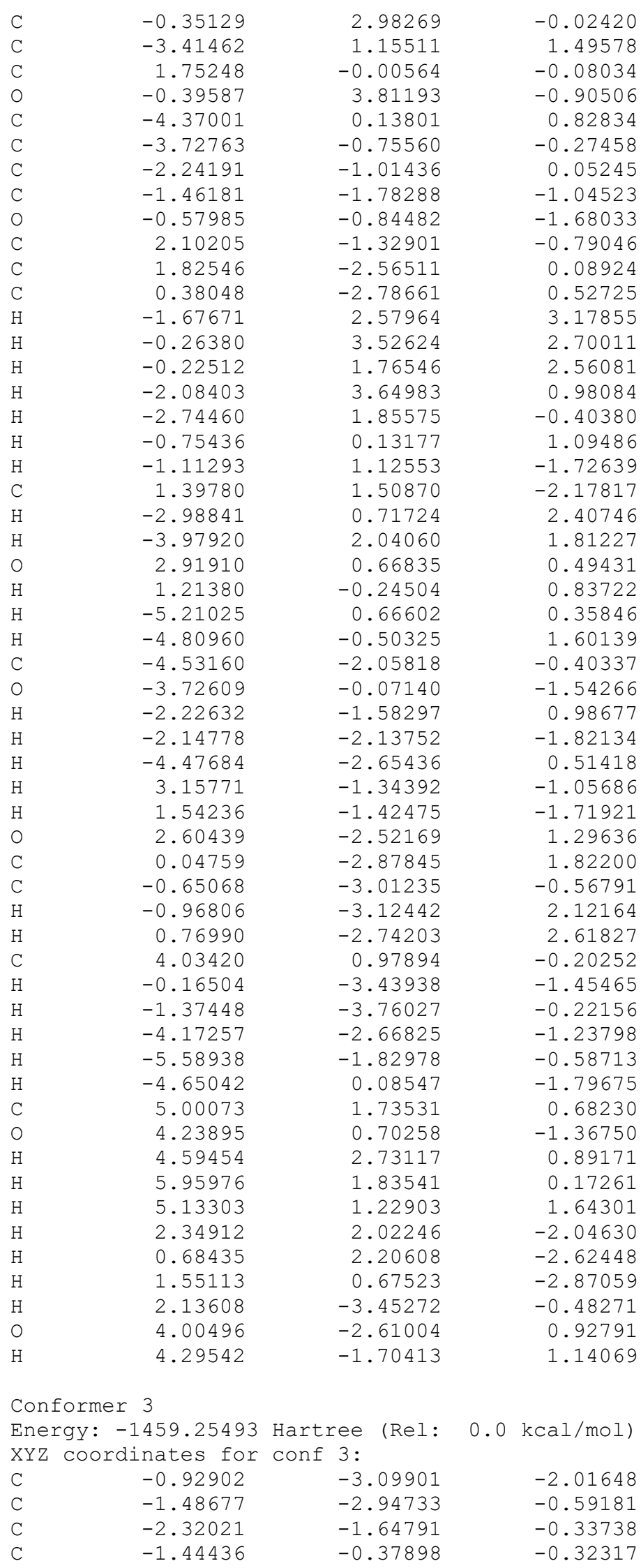




\begin{tabular}{|c|c|c|c|}
\hline $\mathrm{C}$ & -0.65383 & -0.20797 & 0.98764 \\
\hline C & 0.76638 & -0.86219 & 0.97250 \\
\hline 0 & 0.63494 & -2.09575 & 0.19012 \\
\hline C & -0.39624 & -2.96672 & 0.47192 \\
\hline C & -3.48590 & -1.43284 & -1.31215 \\
\hline C & 1.70398 & 0.03330 & 0.10510 \\
\hline 0 & -0.42604 & -3.64371 & 1.47439 \\
\hline C & -4.40114 & -0.28354 & -0.82479 \\
\hline C & -3.68923 & 0.83304 & 0.00151 \\
\hline C & -2.22739 & 0.95608 & -0.46715 \\
\hline C & -1.31981 & 1.95332 & 0.29290 \\
\hline 0 & -0.55756 & 1.18813 & 1.24200 \\
\hline C & 2.30200 & 1.28389 & 0.78274 \\
\hline C & 2.18463 & 2.58565 & -0.05430 \\
\hline C & 0.84906 & 3.32204 & 0.00783 \\
\hline $\mathrm{H}$ & -1.74659 & -3.12928 & -2.74341 \\
\hline $\mathrm{H}$ & -0.36797 & -4.03531 & -2.10988 \\
\hline $\mathrm{H}$ & -0.25583 & -2.28064 & -2.28478 \\
\hline $\mathrm{H}$ & -2.13923 & -3.80001 & -0.37477 \\
\hline $\mathrm{H}$ & -2.75707 & -1.75812 & 0.66219 \\
\hline $\mathrm{H}$ & -0.74569 & -0.43592 & -1.16430 \\
\hline $\mathrm{H}$ & -1.21828 & -0.66234 & 1.81273 \\
\hline C & 1.23015 & -1.15796 & 2.39924 \\
\hline $\mathrm{H}$ & -3.09105 & -1.19415 & -2.30795 \\
\hline $\mathrm{H}$ & -4.07404 & -2.35182 & -1.42359 \\
\hline 0 & 2.72691 & -0.72946 & -0.62531 \\
\hline $\mathrm{H}$ & 1.10383 & 0.35262 & -0.74363 \\
\hline $\mathrm{H}$ & -5.20151 & -0.68774 & -0.19118 \\
\hline $\mathrm{H}$ & -4.89795 & 0.16940 & -1.69081 \\
\hline C & -4.46380 & 2.15119 & -0.14021 \\
\hline O & -3.62181 & 0.46984 & 1.39328 \\
\hline $\mathrm{H}$ & -2.27003 & 1.24346 & -1.52411 \\
\hline $\mathrm{H}$ & -1.89304 & 2.67788 & 0.87895 \\
\hline $\mathrm{H}$ & -4.44758 & 2.51736 & -1.17287 \\
\hline $\mathrm{H}$ & 3.35654 & 1.09856 & 0.99205 \\
\hline $\mathrm{H}$ & 1.82043 & 1.47035 & 1.74242 \\
\hline O & 2.40083 & 2.35505 & -1.46095 \\
\hline $\mathrm{C}$ & 0.78777 & 4.50279 & 0.63237 \\
\hline C & -0.37440 & 2.71864 & -0.66528 \\
\hline $\mathrm{H}$ & -0.14288 & 5.06203 & 0.70017 \\
\hline $\mathrm{H}$ & 1.65648 & 4.94937 & 1.10952 \\
\hline C & 3.78272 & -1.33493 & -0.02168 \\
\hline $\mathrm{H}$ & -0.96090 & 3.51772 & -1.13533 \\
\hline $\mathrm{H}$ & -0.05729 & 2.05754 & -1.47900 \\
\hline $\mathrm{H}$ & -4.04786 & 2.92507 & 0.51211 \\
\hline $\mathrm{H}$ & -5.51440 & 2.00426 & 0.14205 \\
\hline $\mathrm{H}$ & -4.53170 & 0.39514 & 1.72516 \\
\hline C & 4.59033 & -2.10562 & -1.03985 \\
\hline 0 & 4.04383 & -1.25941 & 1.15908 \\
\hline $\mathrm{H}$ & 3.95883 & -2.85998 & -1.51972 \\
\hline $\mathrm{H}$ & 5.43323 & -2.58728 & -0.54345 \\
\hline $\mathrm{H}$ & 4.96008 & -1.43856 & -1.82581 \\
\hline $\mathrm{H}$ & 2.22332 & -1.60329 & 2.41318 \\
\hline $\mathrm{H}$ & 0.52212 & -1.83938 & 2.87604 \\
\hline $\mathrm{H}$ & 1.25416 & -0.23170 & 2.98230 \\
\hline $\mathrm{H}$ & 2.96177 & 3.27214 & 0.30150 \\
\hline 0 & 3.74126 & 1.81930 & -1.63832 \\
\hline $\mathrm{H}$ & 3.54163 & 0.86111 & -1.64707 \\
\hline \multicolumn{4}{|c|}{ Conformer 4} \\
\hline \multicolumn{4}{|c|}{ Energy: -1459.25279 Hartree (Rel: $1.3 \mathrm{kcal} / \mathrm{mol})$} \\
\hline $\mathrm{C}$ & 1.25968 & -2.98756 & 2.13300 \\
\hline
\end{tabular}




\begin{tabular}{|c|c|c|c|}
\hline C & 1.79373 & -2.83455 & 0.69919 \\
\hline C & 2.48028 & -1.46470 & 0.38671 \\
\hline $\mathrm{C}$ & 1.46809 & -0.30350 & 0.31831 \\
\hline $\mathrm{C}$ & 0.66474 & -0.27543 & -0.99639 \\
\hline C & -0.67881 & -1.06971 & -0.93355 \\
\hline O & -0.40863 & -2.24504 & -0.09696 \\
\hline C & 0.70548 & -3.01363 & -0.35279 \\
\hline $\mathrm{C}$ & 3.60932 & -1.07951 & 1.35185 \\
\hline $\mathrm{C}$ & -1.70771 & -0.24307 & -0.09930 \\
\hline 0 & 0.80267 & -3.72674 & -1.32608 \\
\hline C & 4.39304 & 0.14320 & 0.81598 \\
\hline C & 3.56399 & 1.14025 & -0.05301 \\
\hline C & 2.09496 & 1.11484 & 0.41045 \\
\hline C & 1.08584 & 1.97193 & -0.38958 \\
\hline 0 & 0.42839 & 1.09154 & -1.31557 \\
\hline $\mathrm{C}$ & -2.42910 & 0.90067 & -0.85348 \\
\hline $\mathrm{C}$ & -2.46835 & 2.27131 & -0.14105 \\
\hline C & -1.20648 & 3.12991 & -0.17738 \\
\hline $\mathrm{H}$ & 2.07962 & -2.91664 & 2.85437 \\
\hline $\mathrm{H}$ & 0.78864 & -3.96805 & 2.26286 \\
\hline $\mathrm{H}$ & 0.51387 & -2.22689 & 2.37807 \\
\hline $\mathrm{H}$ & 2.53264 & -3.62110 & 0.51119 \\
\hline $\mathrm{H}$ & 2.93183 & -1.56924 & -0.60692 \\
\hline $\mathrm{H}$ & 0.77685 & -0.40531 & 1.16131 \\
\hline $\mathrm{H}$ & 1.27077 & -0.70806 & -1.80361 \\
\hline $\mathrm{C}$ & -1.13383 & -1.48853 & -2.33178 \\
\hline $\mathrm{H}$ & 3.18562 & -0.84433 & 2.33663 \\
\hline $\mathrm{H}$ & 4.29650 & -1.92091 & 1.50269 \\
\hline 0 & -2.62709 & -1.08739 & 0.65209 \\
\hline $\mathrm{H}$ & -1.14455 & 0.17897 & 0.72945 \\
\hline $\mathrm{H}$ & 5.23353 & -0.19591 & 0.19631 \\
\hline $\mathrm{H}$ & 4.83605 & 0.68273 & 1.66139 \\
\hline C & 4.18615 & 2.54094 & 0.03658 \\
\hline O & 3.54339 & 0.71811 & -1.42920 \\
\hline $\mathrm{H}$ & 2.09954 & 1.44618 & 1.45550 \\
\hline $\mathrm{H}$ & 1.57473 & 2.73869 & -0.99780 \\
\hline $\mathrm{H}$ & 4.11935 & 2.94542 & 1.05278 \\
\hline $\mathrm{H}$ & -3.45796 & 0.59387 & -1.04376 \\
\hline $\mathrm{H}$ & -1.96468 & 1.06777 & -1.82508 \\
\hline 0 & -2.86667 & 1.98841 & 1.21521 \\
\hline C & -1.24591 & 4.31541 & -0.79637 \\
\hline $\mathrm{C}$ & 0.04953 & 2.65579 & 0.53493 \\
\hline $\mathrm{H}$ & -0.37948 & 4.97263 & -0.83300 \\
\hline $\mathrm{H}$ & -2.14149 & 4.66899 & -1.30184 \\
\hline $\mathrm{C}$ & -3.68233 & -1.72427 & 0.10954 \\
\hline $\mathrm{H}$ & 0.55054 & 3.51086 & 1.00640 \\
\hline $\mathrm{H}$ & -0.22504 & 1.97634 & 1.35032 \\
\hline $\mathrm{H}$ & 3.69433 & 3.23642 & -0.65046 \\
\hline $\mathrm{H}$ & 5.24931 & 2.49864 & -0.23362 \\
\hline $\mathrm{H}$ & 4.45827 & 0.72472 & -1.75553 \\
\hline C & -4.43726 & -2.47573 & 1.18252 \\
\hline 0 & -3.99515 & -1.69971 & -1.06509 \\
\hline $\mathrm{H}$ & -3.77821 & -3.20917 & 1.65831 \\
\hline $\mathrm{H}$ & -5.29517 & -2.98195 & 0.73864 \\
\hline $\mathrm{H}$ & -4.77424 & -1.78298 & 1.96041 \\
\hline $\mathrm{H}$ & -2.08850 & -2.01248 & -2.29619 \\
\hline $\mathrm{H}$ & -0.37972 & -2.13894 & -2.78015 \\
\hline $\mathrm{H}$ & -1.24487 & -0.60705 & -2.97156 \\
\hline $\mathrm{H}$ & -3.27313 & 2.84878 & -0.60984 \\
\hline 0 & -3.34228 & 3.21914 & 1.81713 \\
\hline $\mathrm{H}$ & -2.51216 & 3.60701 & 2.15423 \\
\hline
\end{tabular}


Briarellin A - revised

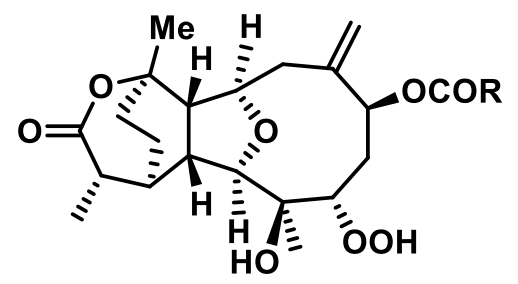

$$
\text { Rel energy (kcal/mol): } \begin{gathered}
\text { Conf } 1 \\
0.0
\end{gathered}
$$

\begin{tabular}{|c|c|c|c|c|c|c|c|c|}
\hline iGau & jGau & Jexp & Jcalc & $\operatorname{diff}$ & & 1 & & \\
\hline 35 & 36 & 3.30 & 4.11 & 0.81 & {[} & $4.11]$ & $\mathrm{H} 1$ & $\mathrm{H} 2$ \\
\hline 40 & 45 & 10.20 & 11.12 & 0.92 & {[} & $11.12]$ & H4 & H5 \\
\hline 40 & 46 & 4. & 3.21 & -0.79 & {[} & $3.21]$ & H4 & H5 \\
\hline 46 & 47 & 10.80 & 11.17 & 0.37 & {[} & 11.17 ] & H5 & $\mathrm{H} 6$ \\
\hline 45 & 47 & 5.60 & 5.51 & -0.09 & {[} & $5.51]$ & H5 & $\mathrm{HC}$ \\
\hline 31 & 33 & 7.50 & 7.59 & 0.09 & {[} & $7.59]$ & $\mathrm{H} 17-\mathrm{Me}$ & \\
\hline & & For Js: & $r m s c$ & $=0.62 \mathrm{~Hz}$ & $\mathrm{~N}=6$ & $\{-0.79$ & $0.92\}$ & \\
\hline
\end{tabular}

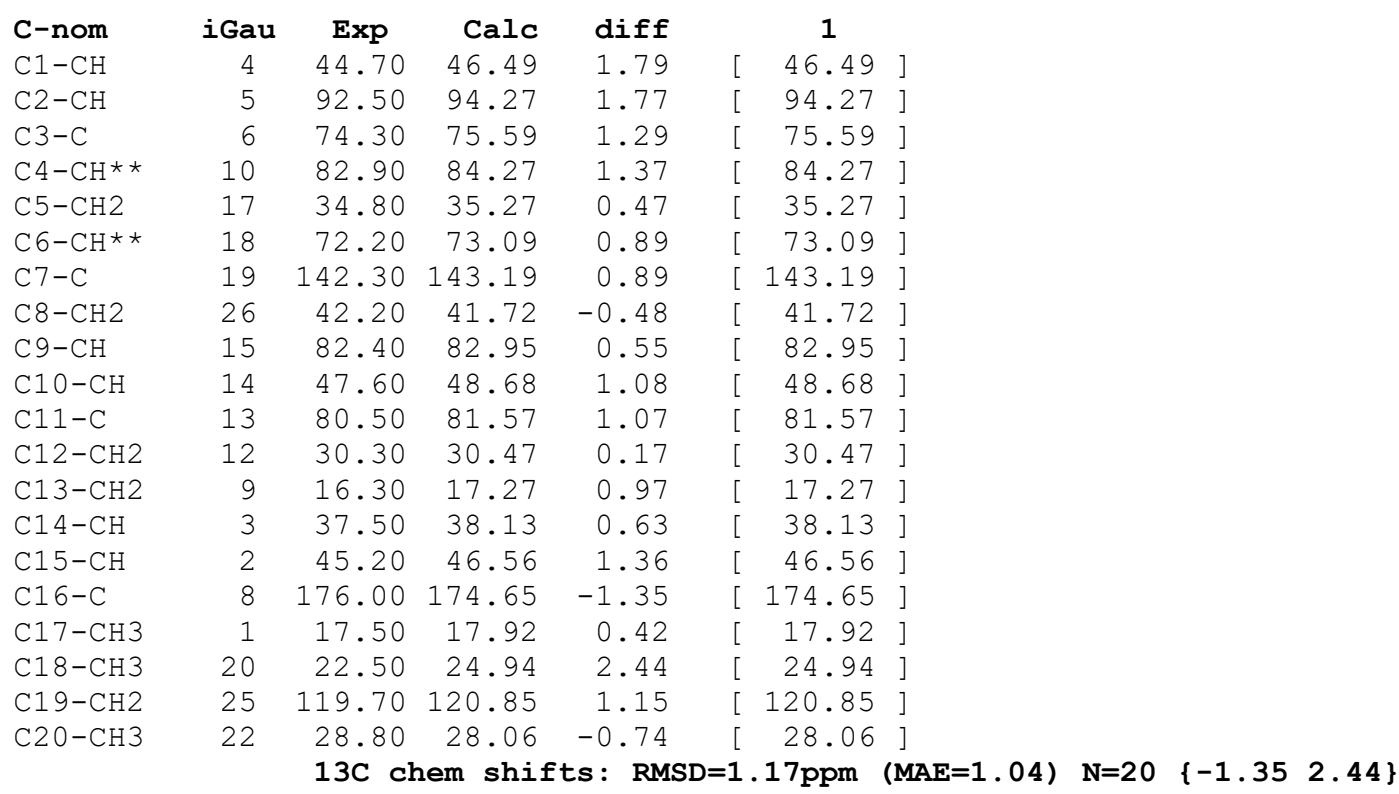

Conformer 1

Energy: -1459.27486 Hartree (Rel: $0.0 \mathrm{kcal} / \mathrm{mol}$ ) XYZ coordinates for conf 1 :

$\begin{array}{lrrr}\text { C } & 5.30475 & 1.41150 & -0.45940 \\ \text { C } & 3.93226 & 1.06487 & 0.15323 \\ \text { C } & 2.82057 & 0.87420 & -0.90035 \\ \text { C } & 1.46403 & 0.54876 & -0.23060 \\ \text { C } & 0.25889 & 0.88600 & -1.14107 \\ \text { C } & -0.73317 & 1.93702 & -0.56689 \\ \text { O } & 0.10284 & 3.07070 & -0.31154 \\ \text { C } & 4.12083 & -0.03500 & 1.20466 \\ \text { C } & 3.15151 & -0.18258 & -1.96396 \\ \text { C } & -1.37487 & 1.47304 & 0.79278\end{array}$




\begin{tabular}{|c|c|c|}
\hline 4.84847 & 0.15215 & 2.16091 \\
\hline 3.38089 & -1.56100 & -1.31461 \\
\hline 2.64788 & -1.72575 & 0.03304 \\
\hline 1.30534 & -0.96449 & 0.09944 \\
\hline 0.22611 & -1.48182 & -0.88409 \\
\hline-0.42468 & -0.33173 & -1.43528 \\
\hline-2.71310 & 0.71937 & 0.78347 \\
\hline-2.74991 & -0.69079 & 0.17362 \\
\hline-1.74919 & -1.68911 & 0.74248 \\
\hline-1.77952 & 2.31541 & -1.62555 \\
\hline-1.47971 & 2.60245 & 1.69465 \\
\hline 2.50800 & -3.20642 & 0.39127 \\
\hline 3.48705 & -1.22730 & 1.13706 \\
\hline-4.08501 & -1.23235 & 0.44856 \\
\hline-1.70223 & -1.95778 & 2.05220 \\
\hline-0.85700 & -2.38900 & -0.25951 \\
\hline-5.05649 & -0.96117 & -0.45095 \\
\hline-6.35773 & -1.61319 & -0.04623 \\
\hline-4.89299 & -0.28475 & -1.44796 \\
\hline 5.72558 & 0.57524 & -1.02778 \\
\hline 5.20673 & 2.26688 & -1.13670 \\
\hline 6.01243 & 1.67214 & 0.33130 \\
\hline 3.63554 & 1.93027 & 0.76174 \\
\hline 2.71369 & 1.84822 & -1.39337 \\
\hline 1.39962 & 1.14432 & 0.68405 \\
\hline 0.62043 & 1.31315 & -2.08705 \\
\hline-0.22803 & 3.47491 & 0.51025 \\
\hline 4.02684 & 0.11105 & -2.55200 \\
\hline 2.31792 & -0.23559 & -2.67510 \\
\hline-0.63646 & 0.88123 & 1.34526 \\
\hline 3.06062 & -2.35707 & -1.99531 \\
\hline 4.44577 & -1.74037 & -1.12554 \\
\hline 0.92785 & -1.07495 & 1.12166 \\
\hline 0.69335 & -2.03820 & -1.70942 \\
\hline-3.46807 & 1.32040 & 0.27116 \\
\hline-3.02011 & 0.63157 & 1.83306 \\
\hline-2.64339 & -0.63487 & -0.90534 \\
\hline-2.43368 & 3.10423 & -1.24510 \\
\hline-1.26361 & 2.69478 & -2.51416 \\
\hline-2.39804 & 1.46357 & -1.92488 \\
\hline-2.55043 & 3.49742 & 1.25626 \\
\hline 3.49541 & -3.67921 & 0.40160 \\
\hline 2.06038 & -3.32601 & 1.38294 \\
\hline 1.88851 & -3.73089 & -0.34215 \\
\hline-1.01548 & -2.69676 & 2.45708 \\
\hline-2.36163 & -1.47108 & 2.76638 \\
\hline-1.47713 & -2.77642 & -1.07947 \\
\hline-0.36973 & -3.25166 & 0.20614 \\
\hline-7.12839 & -1.38110 & -0.78206 \\
\hline-6.22761 & -2.69787 & 0.02640 \\
\hline-6.66707 & -1.25428 & 0.9408 \\
\hline-3.22377 & 3.30929 & 1.9363 \\
\hline
\end{tabular}


Briarellin B - original

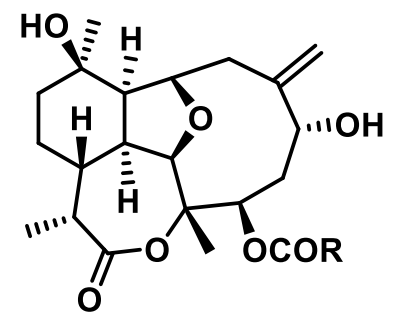

(the best fit to the experimental data still gives unacceptable rmsd $>3$ ppm)

$$
\text { Rel energy (kcal/mol): } \begin{gathered}
\text { Conf } 1 \\
0.0
\end{gathered}
$$

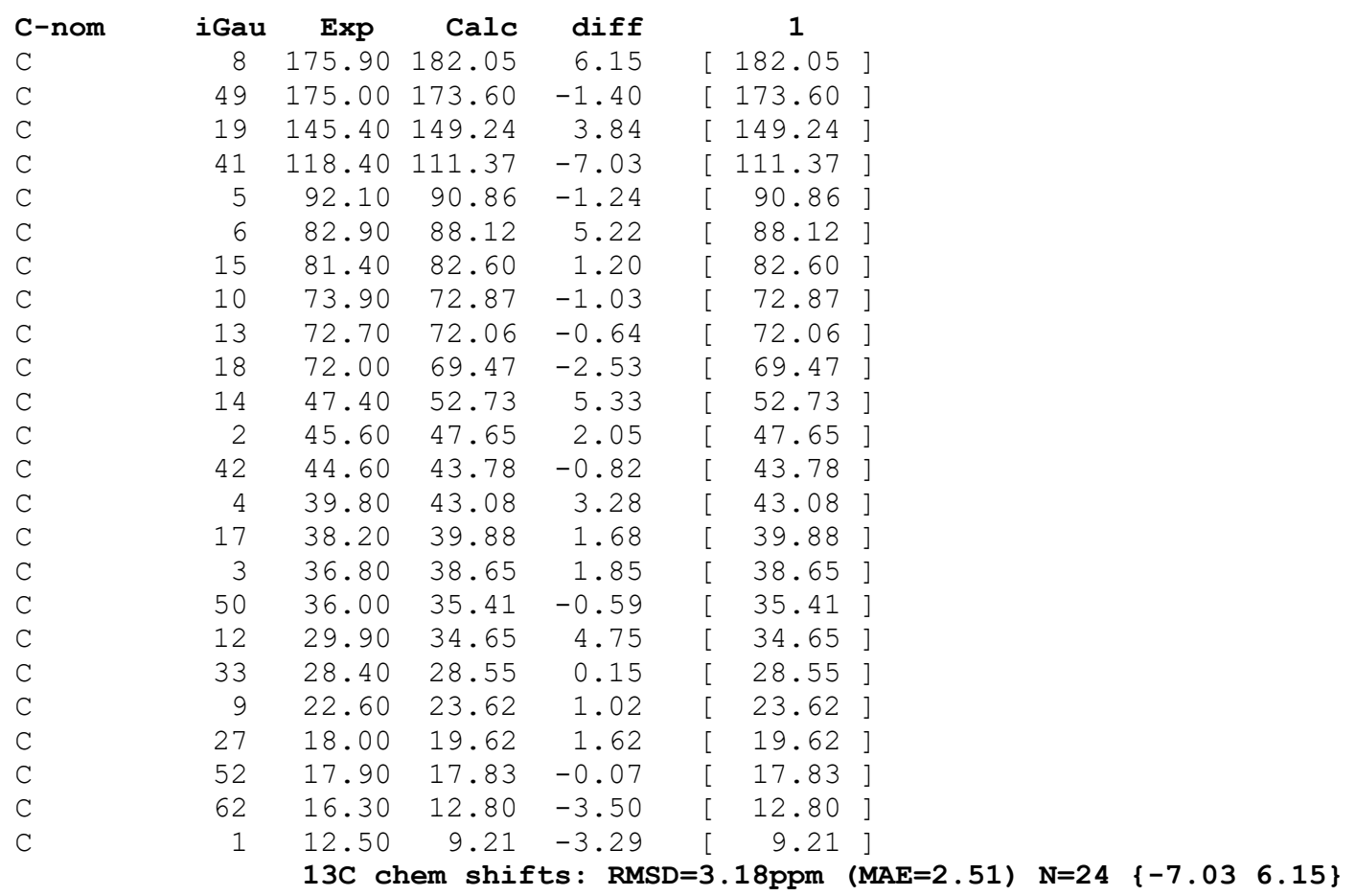

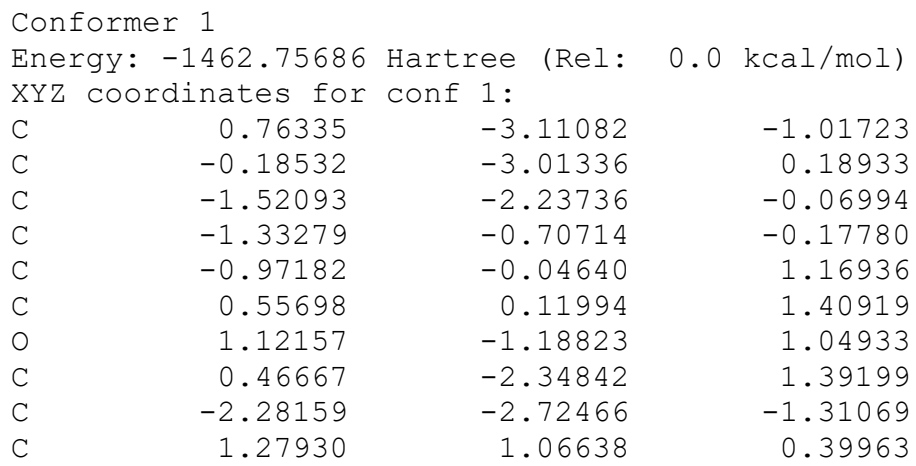




\begin{tabular}{|c|c|c|}
\hline 0.40867 & -2.75422 & 2.53139 \\
\hline-3.70454 & -2.11852 & -1.36875 \\
\hline-3.89257 & -0.79102 & -0.59283 \\
\hline-2.59834 & 0.06169 & -0.66166 \\
\hline-2.64334 & 1.34136 & 0.21589 \\
\hline-1.64756 & 1.19930 & 1.24306 \\
\hline 1.22818 & 2.59392 & 0.57607 \\
\hline-0.09954 & 3.35253 & 0.38132 \\
\hline-0.93748 & 2.91911 & -0.82439 \\
\hline 0.28488 & -3.66328 & -1.83169 \\
\hline 1.67380 & -3.65519 & -0.74195 \\
\hline 1.06544 & -2.12881 & -1.38874 \\
\hline-0.46205 & -4.02490 & 0.50639 \\
\hline-2.15683 & -2.43033 & 0.80049 \\
\hline-0.53728 & -0.51433 & -0.90335 \\
\hline-1.34325 & -0.67739 & 1.99118 \\
\hline 0.87375 & 0.46084 & 2.86283 \\
\hline-1.72152 & -2.44267 & -2.21113 \\
\hline-2.34673 & -3.81933 & -1.31676 \\
\hline 0.96664 & 0.80656 & -0.60986 \\
\hline-4.43436 & -2.81945 & -0.94931 \\
\hline-3.98685 & -1.95857 & -2.41528 \\
\hline-5.10909 & -0.04049 & -1.15464 \\
\hline-4.16300 & -1.17775 & 0.77162 \\
\hline-2.46020 & 0.34758 & -1.71050 \\
\hline-3.61463 & 1.42579 & 0.72158 \\
\hline-5.34568 & 0.85326 & -0.56421 \\
\hline 1.63619 & 2.87205 & 1.55371 \\
\hline 1.93947 & 2.97923 & -0.16682 \\
\hline 0.17086 & 4.76364 & 0.32855 \\
\hline-0.41839 & 2.80975 & -2.05340 \\
\hline-2.40033 & 2.67571 & -0.53375 \\
\hline-1.03584 & 2.56598 & -2.91451 \\
\hline 0.63989 & 2.96225 & -2.25613 \\
\hline-2.99641 & 2.67733 & -1.45336 \\
\hline-2.77313 & 3.49686 & 0.09301 \\
\hline-4.93946 & 0.27754 & -2.18926 \\
\hline-5.98787 & -0.69414 & -1.13435 \\
\hline 3.31026 & 0.16536 & -0.54202 \\
\hline 4.73395 & -0.20618 & -0.18077 \\
\hline 2.78757 & -0.02329 & -1.62344 \\
\hline 5.56665 & -0.67881 & -1.37454 \\
\hline 4.67578 & -0.98895 & 0.58868 \\
\hline 5.19828 & 0.65671 & 0.31335 \\
\hline 1.95315 & 0.56281 & 3.00523 \\
\hline 0.50845 & -0.33083 & 3.52158 \\
\hline 0.38795 & 1.40104 & 3.14435 \\
\hline-0.70481 & 3.23827 & 1.27711 \\
\hline 0.57429 & 4.94878 & -0.53550 \\
\hline-4.42939 & -0.38560 & 1.26556 \\
\hline 2.69411 & 0.74556 & 0.51244 \\
\hline 6.99212 & -1.06666 & -0.96986 \\
\hline 5.59545 & 0.11490 & -2.13124 \\
\hline 5.06486 & -1.53140 & -1.84666 \\
\hline 7.56838 & -1.40629 & -1.83759 \\
\hline 6.98881 & -1.87850 & -0.23209 \\
\hline 7.52595 & -0.21741 & -0.52575 \\
\hline
\end{tabular}


Briarellin B - revised

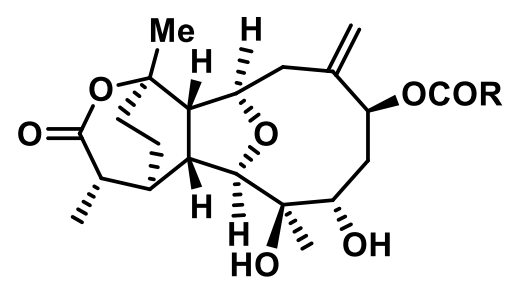

Rel energy (kcal/mol): $\begin{array}{ccc}\text { Conf } 1 & \text { Conf } 2 \\ 0.0 & 2.4\end{array}$

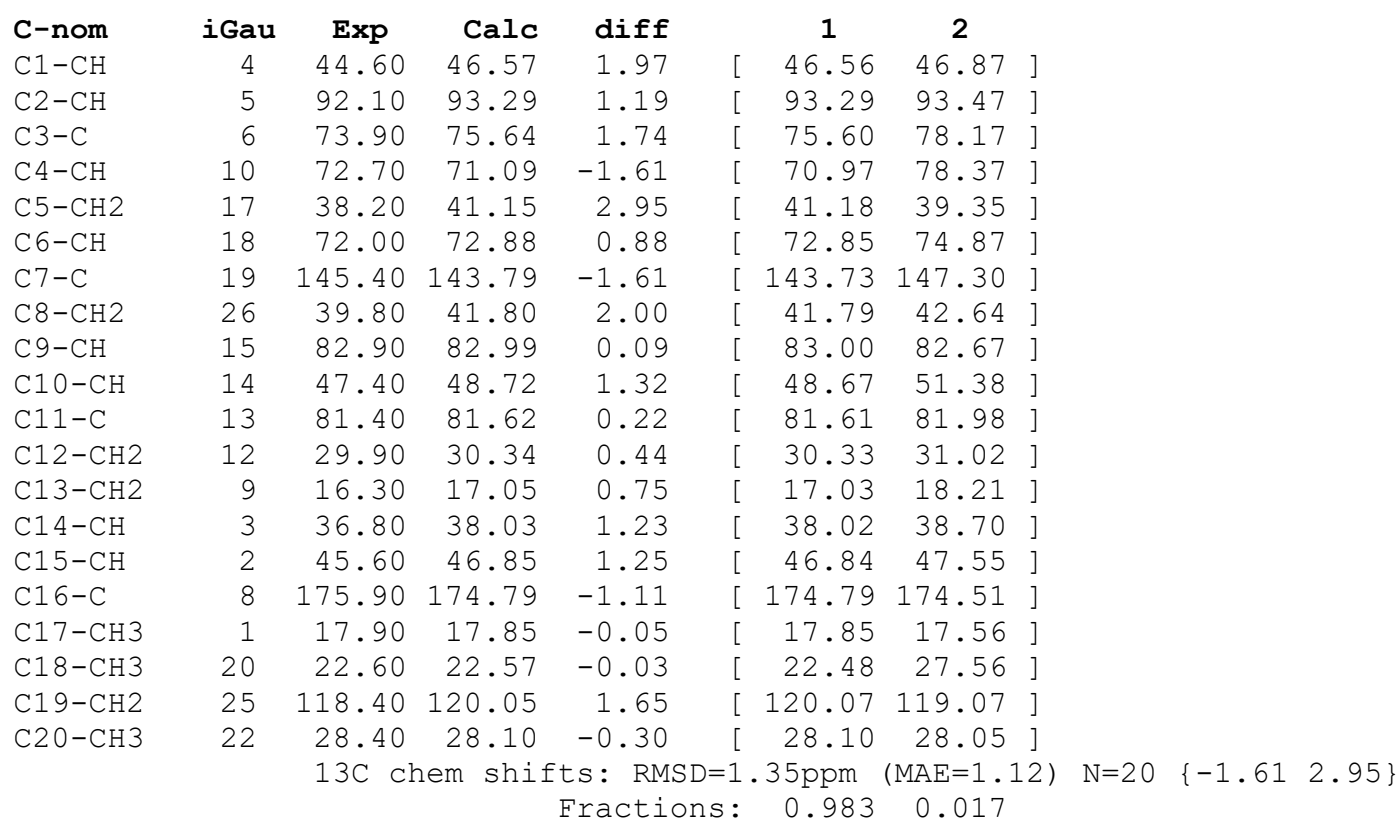

Conformer 1

Energy: -1384.14733 Hartree (Rel: $0.0 \mathrm{kcal} / \mathrm{mol}$ )

XYZ coordinates for conf 1 :

$\begin{array}{lrrr}\text { C } & 5.26433 & 1.36152 & -0.29012 \\ \text { C } & 3.88134 & 0.99100 & 0.28417 \\ \text { C } & 2.75756 & 0.97459 & -0.77472 \\ \text { C } & 1.39503 & 0.62470 & -0.13070 \\ \text { C } & 0.19335 & 1.12055 & -0.97143 \\ \text { C } & -0.73270 & 2.13702 & -0.25233 \\ \text { O } & 0.16800 & 3.18853 & 0.11518 \\ C & 4.03364 & -0.23975 & 1.18672 \\ C & 3.04078 & 0.04577 & -1.96315 \\ C & -1.38003 & 1.57476 & 1.06129 \\ \text { O } & 4.77387 & -0.20249 & 2.15107 \\ C & 3.20712 & -1.41273 & -1.49460 \\ C & 2.48162 & -1.71121 & -0.16717 \\ C & 1.17536 & -0.90857 & 0.01685 \\ C & 0.05986 & -1.25609 & -1.00124 \\ O & -0.54895 & -0.02173 & -1.39400 \\ C & -2.73693 & 0.85070 & 0.97775\end{array}$




\begin{tabular}{|c|c|c|c|}
\hline $\mathrm{C}$ & -2.85637 & -0.47133 & 0.20561 \\
\hline C & -1.89920 & -1.58006 & 0.62594 \\
\hline C & -1.78817 & 2.69871 & -1.21541 \\
\hline O & -1.59470 & 2.74402 & 1.88443 \\
\hline C & 2.28011 & -3.21757 & 0.00703 \\
\hline O & 3.35604 & -1.39030 & 0.97562 \\
\hline 0 & -4.21384 & -0.97814 & 0.44565 \\
\hline C & -1.85310 & -2.01830 & 1.88911 \\
\hline C & -1.05237 & -2.18706 & -0.47150 \\
\hline C & -5.18678 & -0.54990 & -0.38738 \\
\hline C & -6.51710 & -1.16347 & -0.01728 \\
\hline O & -5.00737 & 0.22599 & -1.30645 \\
\hline $\mathrm{H}$ & 5.65699 & 0.58500 & -0.95526 \\
\hline $\mathrm{H}$ & 5.19466 & 2.29409 & -0.86050 \\
\hline $\mathrm{H}$ & 5.98071 & 1.50209 & 0.52299 \\
\hline $\mathrm{H}$ & 3.61898 & 1.78304 & 0.99904 \\
\hline $\mathrm{H}$ & 2.68698 & 2.00540 & -1.14330 \\
\hline $\mathrm{H}$ & 1.36510 & 1.10662 & 0.84959 \\
\hline $\mathrm{H}$ & 0.56094 & 1.64121 & -1.86722 \\
\hline $\mathrm{H}$ & -0.22671 & 3.58145 & 0.91741 \\
\hline $\mathrm{H}$ & 3.92864 & 0.37187 & -2.51397 \\
\hline $\mathrm{H}$ & 2.20586 & 0.11944 & -2.67084 \\
\hline $\mathrm{H}$ & -0.64935 & 0.92564 & 1.55737 \\
\hline $\mathrm{H}$ & 2.83959 & -2.09987 & -2.26440 \\
\hline $\mathrm{H}$ & 4.26337 & -1.66604 & -1.34689 \\
\hline $\mathrm{H}$ & 0.80883 & -1.12763 & 1.02553 \\
\hline $\mathrm{H}$ & 0.49129 & -1.72598 & -1.89714 \\
\hline $\mathrm{H}$ & -3.47340 & 1.54804 & 0.56750 \\
\hline $\mathrm{H}$ & -3.03846 & 0.64972 & 2.01414 \\
\hline $\mathrm{H}$ & -2.76796 & -0.29235 & -0.86149 \\
\hline $\mathrm{H}$ & -2.39784 & 3.45278 & -0.70545 \\
\hline $\mathrm{H}$ & -1.28408 & 3.18427 & -2.05740 \\
\hline $\mathrm{H}$ & -2.45337 & 1.92379 & -1.60761 \\
\hline $\mathrm{H}$ & -1.58625 & 2.47060 & 2.81437 \\
\hline $\mathrm{H}$ & 3.24541 & -3.73018 & -0.05647 \\
\hline $\mathrm{H}$ & 1.83720 & -3.44078 & 0.98283 \\
\hline $\mathrm{H}$ & 1.63028 & -3.61895 & -0.77602 \\
\hline $\mathrm{H}$ & -1.19930 & -2.83503 & 2.18481 \\
\hline $\mathrm{H}$ & -2.48407 & -1.59920 & 2.66891 \\
\hline $\mathrm{H}$ & -1.70080 & -2.44364 & -1.32059 \\
\hline $\mathrm{H}$ & -0.59702 & -3.12051 & -0.12563 \\
\hline $\mathrm{H}$ & -7.27954 & -0.84078 & -0.72714 \\
\hline $\mathrm{H}$ & -6.44378 & -2.25561 & -0.01797 \\
\hline $\mathrm{H}$ & -6.80151 & -0.85503 & 0.99439 \\
\hline
\end{tabular}

Conformer 2

$\begin{array}{lrrr}\text { Energy: -1384.14348 Hartree (Rel: } & 2.4 & \mathrm{kcal} / \mathrm{mol}) \\ \text { XYZ coordinates for conf } 2 \text { : } & & \\ \text { C } & 5.47510 & 0.71164 & -0.59254 \\ \text { C } & 4.12102 & 0.62602 & 0.13966 \\ \text { C } & 2.89775 & 0.79228 & -0.79036 \\ \text { C } & 1.58374 & 0.67947 & 0.02361 \\ \text { C } & 0.38096 & 1.41041 & -0.60642 \\ \text { C } & -0.40300 & 2.32499 & 0.39011 \\ \text { O } & -0.43389 & 1.77328 & 1.71262 \\ \text { C } & 4.13652 & -0.58486 & 1.08265 \\ \text { C } & 2.88844 & -0.19052 & -1.96955 \\ \text { C } & -1.89776 & 2.51682 & -0.03906 \\ \text { O } & 4.96256 & -0.65230 & 1.97258 \\ \text { C } & 2.89331 & -1.64475 & -1.46119 \\ \text { C } & 2.24071 & -1.79906 & -0.07048 \\ \text { C } & 1.09618 & -0.79137 & 0.17151 \\ \text { C } & -0.08159 & -0.93087 & -0.82344\end{array}$




\begin{tabular}{|c|c|c|}
\hline-0.47451 & 0.40509 & -1.17429 \\
\hline-2.95936 & 1.58306 & 0.58944 \\
\hline-3.10326 & 0.14263 & 0.07471 \\
\hline-2.17674 & -0.91871 & 0.66549 \\
\hline 0.28818 & 3.69681 & 0.41202 \\
\hline-2.02029 & 2.61453 & -1.45536 \\
\hline 1.81926 & -3.24942 & 0.16937 \\
\hline 3.24148 & -1.59269 & 0.99217 \\
\hline-4.49476 & -0.20213 & 0.41213 \\
\hline-2.20697 & -1.20257 & 1.97120 \\
\hline-1.31832 & -1.69516 & -0.30798 \\
\hline-5.07420 & -1.19223 & -0.29186 \\
\hline-6.48685 & -1.43880 & 0.18514 \\
\hline-4.52498 & -1.80746 & -1.18660 \\
\hline 5.63687 & -0.13903 & -1.26270 \\
\hline 5.52165 & 1.62846 & -1.19033 \\
\hline 6.29067 & 0.72801 & 0.13456 \\
\hline 4.09377 & 1.47157 & 0.84090 \\
\hline 2.96548 & 1.81298 & -1.18894 \\
\hline 1.77072 & 1.09047 & 1.01954 \\
\hline 0.70535 & 2.04166 & -1.44187 \\
\hline 0.31512 & 2.13212 & 2.21332 \\
\hline 3.74308 & -0.01662 & -2.63091 \\
\hline 1.99389 & -0.00128 & -2.57488 \\
\hline-2.17585 & 3.51212 & 0.32681 \\
\hline 2.37678 & -2.29594 & -2.17416 \\
\hline 3.91441 & -2.03655 & -1.38572 \\
\hline 0.71765 & -0.93813 & 1.18767 \\
\hline 0.25587 & -1.42400 & -1.74439 \\
\hline-3.92077 & 2.06542 & 0.37903 \\
\hline-2.82746 & 1.58184 & 1.67421 \\
\hline-3.03666 & 0.12141 & -1.01343 \\
\hline-0.14480 & 4.32081 & 1.20152 \\
\hline 1.36586 & 3.60282 & 0.59963 \\
\hline 0.15540 & 4.21070 & -0.54520 \\
\hline-1.73944 & 1.75165 & -1.80971 \\
\hline 2.68307 & -3.91119 & 0.04877 \\
\hline 1.42666 & -3.37743 & 1.18296 \\
\hline 1.05227 & -3.55724 & -0.54722 \\
\hline-1.59681 & -1.99594 & 2.39560 \\
\hline-2.84802 & -0.66306 & 2.66364 \\
\hline-1.94038 & -1.96420 & -1.16939 \\
\hline-0.98442 & -2.63254 & 0.14923 \\
\hline-6.98589 & -2.13488 & -0.49016 \\
\hline-6.46265 & -1.86595 & 1.19397 \\
\hline-7.04501 & -0.49951 & 0.23927 \\
\hline
\end{tabular}


Briarellin D - original

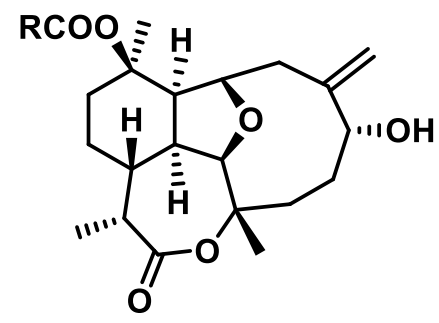

\begin{tabular}{|c|c|c|c|c|c|c|c|}
\hline \multirow[b]{2}{*}{ C-nom } & \multirow[b]{2}{*}{ iGau } & \multicolumn{6}{|c|}{$\begin{array}{c}\text { Conf } 1 \\
0.0\end{array}$} \\
\hline & & $\operatorname{Exp}$ & Calc & $\operatorname{diff}$ & 1 & & \\
\hline $\mathrm{C}-\mathrm{C}$ & 8 & 176.10 & 182.16 & 6.06 & [ 182.16 & ] & \\
\hline$C-C$ & 19 & 147.90 & 147.98 & 0.08 & [ 147.98 & ] & \\
\hline $\mathrm{C}-\mathrm{C}$ & 6 & 84.90 & 88.33 & 3.43 & [ 88.33 & ] & \\
\hline $\mathrm{C}-\mathrm{C}$ & 13 & 81.00 & 84.24 & 3.24 & 84.24 & ] & \\
\hline $\mathrm{C}-\mathrm{CH}$ & 5 & 91.10 & 90.37 & -0.73 & 90.37 & ] & \\
\hline $\mathrm{C}-\mathrm{CH}$ & 15 & 82.40 & 82.30 & -0.10 & 82.30 & ] & \\
\hline $\mathrm{C}-\mathrm{CH}$ & 18 & 72.60 & 71.84 & -0.76 & 71.84 & ] & \\
\hline $\mathrm{C}-\mathrm{CH}$ & 14 & 47.70 & 54.07 & 6.37 & 54.07 & ] & \\
\hline $\mathrm{C}-\mathrm{CH}$ & 2 & 45.90 & 47.34 & 1.44 & 47.34 & ] & \\
\hline $\mathrm{C}-\mathrm{CH}$ & 4 & 44.90 & 42.19 & -2.71 & 42.19 & ] & \\
\hline $\mathrm{C}-\mathrm{CH}$ & 3 & 37.00 & 38.56 & 1.56 & {$[\quad 38.56$} & ] & \\
\hline $\mathrm{C}-\mathrm{CH} 2$ & 42 & 117.30 & 114.93 & -2.37 & [ 114.93 & ] & \\
\hline $\mathrm{C}-\mathrm{CH} 2$ & 43 & 41.40 & 44.35 & 2.95 & {$[44.35$} & ] & \\
\hline $\mathrm{C}-\mathrm{CH} 2$ & 12 & 32.80 & 29.35 & -3.45 & 29.35 & ] & \\
\hline $\mathrm{C}-\mathrm{CH} 2$ & 10 & 30.30 & 33.55 & 3.25 & 33.55 & ] & \\
\hline $\mathrm{C}-\mathrm{CH} 2$ & 17 & 28.70 & 31.49 & 2.79 & 31.49 & ] & \\
\hline $\mathrm{C}-\mathrm{CH} 2$ & 9 & 16.80 & 23.01 & 6.21 & 23.01 & ] & \\
\hline $\mathrm{C}-\mathrm{CH} 3$ & 34 & 28.90 & 23.94 & -4.96 & 23.94 & ] & \\
\hline $\mathrm{C}-\mathrm{CH} 3$ & 27 & 20.70 & 23.10 & 2.40 & 23.10 & ] & \\
\hline $\mathrm{C}-\mathrm{CH} 3$ & 1 & 17.50 & 9.62 & -7.88 & 9.62 & ] & \\
\hline
\end{tabular}

$\begin{array}{lrrr}\text { Conformer } 1 & \\ \text { Energy: }-1387.54498 & \text { Hartree (Rel: } & 0.0 & \mathrm{kcal} / \mathrm{mol}) \\ \text { XYZ coordinates for } & \text { conf } 1: & \\ \text { C } & -0.80071 & 3.45181 & -2.23032 \\ \text { C } & 0.16192 & 3.03426 & -1.10600 \\ \text { C } & 0.69770 & 1.56443 & -1.20155 \\ \text { C } & -0.36153 & 0.50960 & -0.81837 \\ \text { C } & -0.70498 & 0.50253 & 0.68447 \\ \text { C } & -1.94644 & 1.36188 & 1.03931 \\ \text { O } & -1.72441 & 2.64765 & 0.33789 \\ \text { C } & -0.46325 & 3.18461 & 0.27536 \\ \text { C } & 1.26592 & 1.20985 & -2.58248 \\ \text { C } & -3.27347 & 0.88867 & 0.40484 \\ \text { O } & 0.08427 & 3.69478 & 1.22854 \\ \text { C } & 1.97512 & -0.16766 & -2.57937 \\ \text { C } & 1.53543 & -1.14571 & -1.46033 \\ \text { C } & 0.03496 & -0.96116 & -1.13696 \\ \text { C } & -0.45701 & -1.77890 & 0.08904 \\ \text { O } & -0.90895 & -0.84336 & 1.08077 \\ \text { C } & -4.11074 & -0.23150 & 1.04242 \\ \text { C } & -3.62107 & -1.69248 & 1.01192 \\ \text { C } & -2.97871 & -2.16770 & -0.29831 \\ \text { H } & -0.28692 & 3.44054 & -3.19626\end{array}$




\begin{tabular}{|c|c|c|c|}
\hline $\mathrm{H}$ & -1.16610 & 4.47086 & -2.06241 \\
\hline $\mathrm{H}$ & -1.67108 & 2.79342 & -2.29158 \\
\hline $\mathrm{H}$ & 1.03541 & 3.69529 & -1.12510 \\
\hline $\mathrm{H}$ & 1.51532 & 1.49961 & -0.47454 \\
\hline $\mathrm{H}$ & -1.26630 & 0.72711 & -1.39191 \\
\hline $\mathrm{H}$ & 0.14836 & 0.90053 & 1.25535 \\
\hline C & -2.05935 & 1.60170 & 2.54264 \\
\hline $\mathrm{H}$ & 0.44693 & 1.20006 & -3.31216 \\
\hline $\mathrm{H}$ & 1.96849 & 1.98230 & -2.91730 \\
\hline $\mathrm{H}$ & -3.90836 & 1.78292 & 0.39933 \\
\hline $\mathrm{H}$ & -3.09887 & 0.64351 & -0.64680 \\
\hline $\mathrm{H}$ & 3.05768 & -0.04694 & -2.50231 \\
\hline $\mathrm{H}$ & 1.79505 & -0.66607 & -3.53884 \\
\hline C & 1.85491 & -2.59501 & -1.84901 \\
\hline O & 2.20254 & -0.80811 & -0.18509 \\
\hline $\mathrm{H}$ & -0.50267 & -1.28545 & -2.03486 \\
\hline $\mathrm{H}$ & 0.38516 & -2.32938 & 0.52183 \\
\hline $\mathrm{H}$ & 1.68328 & -3.27877 & -1.01154 \\
\hline $\mathrm{H}$ & -4.35028 & 0.01184 & 2.08435 \\
\hline $\mathrm{H}$ & -5.07050 & -0.22895 & 0.50995 \\
\hline 0 & -4.81050 & -2.45298 & 1.30372 \\
\hline C & -3.64857 & -2.13213 & -1.45628 \\
\hline $\mathrm{C}$ & -1.59890 & -2.78534 & -0.18480 \\
\hline $\mathrm{H}$ & -3.22362 & -2.52430 & -2.37751 \\
\hline $\mathrm{H}$ & -4.65649 & -1.72929 & -1.52054 \\
\hline $\mathrm{H}$ & -1.35507 & -3.35942 & -1.08650 \\
\hline $\mathrm{H}$ & -1.61200 & -3.50233 & 0.64872 \\
\hline $\mathrm{H}$ & 1.20909 & -2.90183 & -2.67871 \\
\hline $\mathrm{H}$ & 2.89547 & -2.69199 & -2.16196 \\
\hline C & 3.53860 & -0.89208 & -0.00666 \\
\hline $\mathrm{C}$ & 3.90347 & -0.47955 & 1.40854 \\
\hline 0 & 4.33717 & -1.23743 & -0.85621 \\
\hline C & 5.38617 & -0.67048 & 1.73595 \\
\hline $\mathrm{H}$ & 3.26737 & -1.04412 & 2.10234 \\
\hline $\mathrm{H}$ & 3.61006 & 0.57236 & 1.53352 \\
\hline $\mathrm{H}$ & -2.93373 & 2.22065 & 2.76971 \\
\hline $\mathrm{H}$ & -1.16775 & 2.11369 & 2.91415 \\
\hline $\mathrm{H}$ & -2.15979 & 0.64772 & 3.07072 \\
\hline $\mathrm{H}$ & -2.89506 & -1.84350 & 1.81623 \\
\hline $\mathrm{H}$ & -4.56232 & -3.39135 & 1.30242 \\
\hline $\mathrm{C}$ & 5.72701 & -0.22794 & 3.16238 \\
\hline $\mathrm{H}$ & 5.98886 & -0.10761 & 1.01371 \\
\hline $\mathrm{H}$ & 5.65349 & -1.72531 & 1.59819 \\
\hline $\mathrm{H}$ & 6.79158 & -0.37561 & 3.37519 \\
\hline $\mathrm{H}$ & 5.15560 & -0.79912 & 3.90443 \\
\hline $\mathrm{H}$ & 5.49994 & 0.83430 & 3.31505 \\
\hline
\end{tabular}


Briarellin D - revised

Note: briarellin D has the same core as "K";

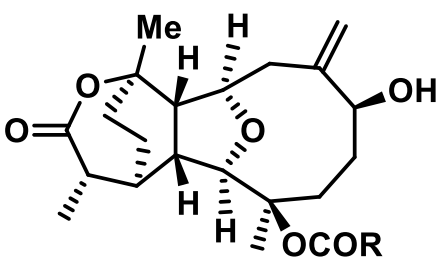

Rel energy (kcal/mol): $\begin{array}{ccccc}\text { Conf } 1 & \text { Conf } 2 & \text { Conf } 3 & \text { Conf } 4 & \text { Conf } 5 \\ 0.8 & 0.3 & 0.7 & 0.4 & 0.0\end{array}$

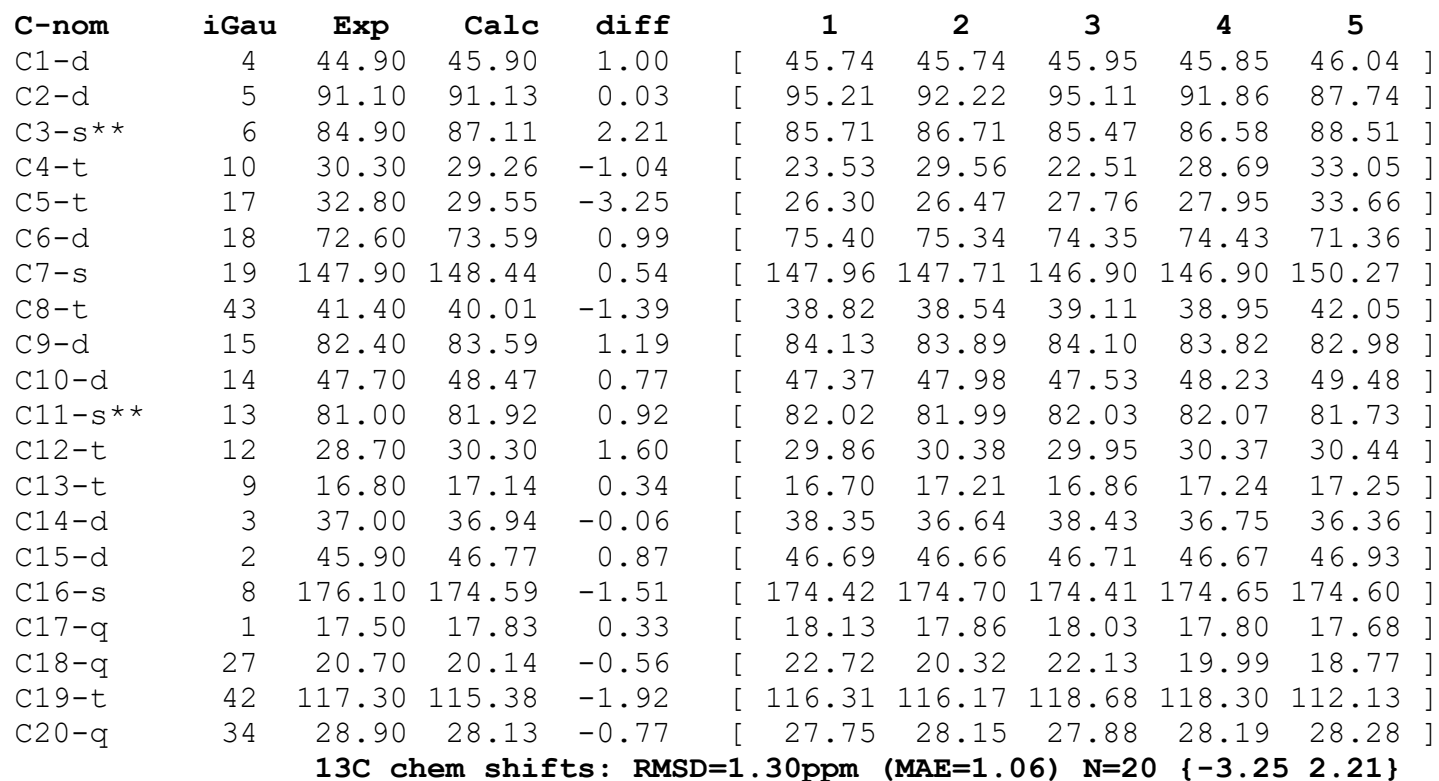

Fractions: $0.103 \quad 0.223 \quad 0.114 \quad 0.189 \quad 0.371$

** carbons C3 and C11 are misassigned (the chem shift values should be switched)

Conformer 1

Energy: -1308.92839 Hartree (Rel: $0.8 \mathrm{kcal} / \mathrm{mol}$ )

XYZ coordinates for conf 1 :

$\begin{array}{lrrr}\text { C } & 4.01660 & 2.42074 & 0.09998 \\ \text { C } & 2.79854 & 1.56255 & 0.50014 \\ \text { C } & 1.85843 & 1.24790 & -0.68295 \\ \text { C } & 0.62424 & 0.43379 & -0.22438 \\ \text { C } & -0.54016 & 0.53635 & -1.24021 \\ \text { C } & -1.88035 & 1.12213 & -0.69322 \\ \text { O } & 3.06592 & -0.89722 & 1.03831 \\ \text { C } & 3.28466 & 0.39432 & 1.36569 \\ \text { C } & 2.54827 & 0.54145 & -1.85907 \\ \text { C } & -2.57035 & 0.19140 & 0.32549 \\ \text { O } & 3.88392 & 0.61078 & 2.40172 \\ C & 3.12278 & -0.82413 & -1.43250 \\ C & 2.43116 & -1.41330 & -0.18872 \\ \text { C } & 0.92391 & -1.08929 & -0.09440 \\ C & 0.04663 & -1.77859 & -1.16981\end{array}$




\begin{tabular}{|c|c|c|c|}
\hline 0 & -0.76740 & -0.76657 & -1.77350 \\
\hline $\mathrm{C}$ & -3.30106 & -1.01478 & -0.31410 \\
\hline C & -3.11229 & -2.34616 & 0.42344 \\
\hline $\mathrm{C}$ & -1.65516 & -2.77551 & 0.58848 \\
\hline $\mathrm{H}$ & 3.68460 & 3.31566 & -0.43746 \\
\hline $\mathrm{H}$ & 4.56415 & 2.73248 & 0.99262 \\
\hline $\mathrm{H}$ & 4.70973 & 1.87347 & -0.54783 \\
\hline $\mathrm{H}$ & 2.21265 & 2.16261 & 1.21030 \\
\hline $\mathrm{H}$ & 1.49373 & 2.22306 & -1.03038 \\
\hline $\mathrm{H}$ & 0.29781 & 0.82695 & 0.74156 \\
\hline $\mathrm{H}$ & -0.24163 & 1.18664 & -2.07435 \\
\hline $\mathrm{C}$ & -2.77835 & 1.51534 & -1.87285 \\
\hline $\mathrm{H}$ & 1.81251 & 0.40120 & -2.65994 \\
\hline $\mathrm{H}$ & 3.33651 & 1.17094 & -2.28364 \\
\hline $\mathrm{H}$ & -1.81972 & -0.15767 & 1.04408 \\
\hline $\mathrm{H}$ & -3.28539 & 0.78853 & 0.89391 \\
\hline $\mathrm{H}$ & 4.19253 & -0.75426 & -1.20358 \\
\hline $\mathrm{H}$ & 3.03868 & -1.54040 & -2.25704 \\
\hline C & 2.71830 & -2.91253 & -0.07442 \\
\hline 0 & -1.40350 & 2.33715 & -0.00834 \\
\hline $\mathrm{H}$ & 0.60064 & -1.42755 & 0.89224 \\
\hline $\mathrm{H}$ & 0.67292 & -2.19044 & -1.97347 \\
\hline $\mathrm{H}$ & 2.30863 & -3.31686 & 0.85632 \\
\hline $\mathrm{H}$ & -4.37889 & -0.82308 & -0.35611 \\
\hline $\mathrm{H}$ & -2.96489 & -1.16763 & -1.33872 \\
\hline 0 & -3.80754 & -2.24379 & 1.66299 \\
\hline C & -1.15408 & -3.04866 & 1.80034 \\
\hline C & -0.82379 & -2.96923 & -0.66992 \\
\hline $\mathrm{H}$ & -0.14650 & -3.43515 & 1.92970 \\
\hline $\mathrm{H}$ & -1.73165 & -2.89156 & 2.70566 \\
\hline $\mathrm{H}$ & -0.13463 & -3.80626 & -0.50743 \\
\hline $\mathrm{H}$ & -1.46817 & -3.26011 & -1.50967 \\
\hline $\mathrm{H}$ & 2.28907 & -3.46152 & -0.91752 \\
\hline $\mathrm{H}$ & 3.79971 & -3.08330 & -0.07352 \\
\hline C & -2.24343 & 3.24405 & 0.53450 \\
\hline C & -1.45540 & 4.36358 & 1.17909 \\
\hline 0 & -3.45620 & 3.17498 & 0.51936 \\
\hline $\mathrm{H}$ & -2.14141 & 5.12534 & 1.55140 \\
\hline $\mathrm{H}$ & -0.86029 & 3.96927 & 2.00993 \\
\hline $\mathrm{H}$ & -0.76065 & 4.80598 & 0.45832 \\
\hline $\mathrm{H}$ & -3.76881 & 1.82659 & -1.53841 \\
\hline $\mathrm{H}$ & -2.31863 & 2.34229 & -2.42521 \\
\hline $\mathrm{H}$ & -2.88132 & 0.67176 & -2.56085 \\
\hline $\mathrm{H}$ & -3.59451 & -3.11940 & -0.20206 \\
\hline $\mathrm{H}$ & -3.79289 & -3.11878 & 2.08321 \\
\hline
\end{tabular}

Conformer 2 XYZ coordinates for conf 2:

\begin{tabular}{lcrr} 
XYZ Coordinates for Conf & \multicolumn{1}{l}{ : } & \\
C & 4.51713 & 0.87211 & 0.09242 \\
C & 3.07952 & 0.52009 & 0.52713 \\
C & 2.04758 & 0.63732 & -0.61495 \\
C & 0.62128 & 0.28278 & -0.12165 \\
C & -0.47483 & 0.88381 & -1.03652 \\
C & -1.55463 & 1.77066 & -0.32984 \\
O & 2.43760 & -1.89008 & 0.96203 \\
C & 3.11409 & -0.78209 & 1.33568 \\
C & 2.39648 & -0.19593 & -1.85590 \\
C & -2.52134 & 0.95180 & 0.55136 \\
O & 3.75913 & -0.84569 & 2.36497 \\
C & 2.49686 & -1.69185 & -1.50488 \\
C & 1.65483 & -2.07899 & -0.27306 \\
C & 0.35700 & -1.25399 & -0.12222
\end{tabular}




\begin{tabular}{|c|c|c|c|}
\hline C & -0.68418 & -1.49246 & -1.24442 \\
\hline O & -1.12983 & -0.20744 & -1.69268 \\
\hline $\mathrm{C}$ & -3.61054 & 0.16839 & -0.21671 \\
\hline C & -3.88465 & -1.23751 & 0.33250 \\
\hline $\mathrm{C}$ & -2.64512 & -2.12537 & 0.41083 \\
\hline $\mathrm{H}$ & 4.53048 & 1.84918 & -0.40267 \\
\hline $\mathrm{H}$ & 5.17232 & 0.91416 & 0.96601 \\
\hline $\mathrm{H}$ & 4.92886 & 0.13339 & -0.60348 \\
\hline $\mathrm{H}$ & 2.79177 & 1.26451 & 1.28239 \\
\hline $\mathrm{H}$ & 2.04377 & 1.69553 & -0.89864 \\
\hline $\mathrm{H}$ & 0.50080 & 0.67118 & 0.89397 \\
\hline $\mathrm{H}$ & -0.01431 & 1.49991 & -1.81394 \\
\hline C & -2.30437 & 2.63840 & -1.34600 \\
\hline $\mathrm{H}$ & 1.61579 & -0.03919 & -2.61005 \\
\hline $\mathrm{H}$ & 3.32877 & 0.15244 & -2.31173 \\
\hline $\mathrm{H}$ & -1.93323 & 0.27005 & 1.17647 \\
\hline $\mathrm{H}$ & -2.99183 & 1.65742 & 1.24312 \\
\hline $\mathrm{H}$ & 3.53208 & -1.98300 & -1.29145 \\
\hline $\mathrm{H}$ & 2.18387 & -2.30249 & -2.35865 \\
\hline $\mathrm{C}$ & 1.40288 & -3.58814 & -0.25059 \\
\hline 0 & -0.91424 & 2.65745 & 0.66629 \\
\hline $\mathrm{H}$ & -0.08308 & -1.53875 & 0.83635 \\
\hline $\mathrm{H}$ & -0.20871 & -1.97571 & -2.10990 \\
\hline $\mathrm{H}$ & 0.88871 & -3.88408 & 0.66902 \\
\hline $\mathrm{H}$ & -4.56055 & 0.71331 & -0.19195 \\
\hline $\mathrm{H}$ & -3.33337 & 0.05611 & -1.26391 \\
\hline 0 & -4.53899 & -1.07863 & 1.58851 \\
\hline C & -2.27954 & -2.69560 & 1.56621 \\
\hline C & -1.89279 & -2.40406 & -0.87940 \\
\hline $\mathrm{H}$ & -1.44545 & -3.39005 & 1.62305 \\
\hline $\mathrm{H}$ & -2.79647 & -2.48333 & 2.49665 \\
\hline $\mathrm{H}$ & -1.50691 & -3.42911 & -0.84268 \\
\hline $\mathrm{H}$ & -2.58021 & -2.36639 & -1.73450 \\
\hline $\mathrm{H}$ & 0.80012 & -3.89839 & -1.10897 \\
\hline $\mathrm{H}$ & 2.35744 & -4.12247 & -0.29515 \\
\hline C & 0.01706 & 3.57309 & 0.33967 \\
\hline C & 0.36182 & 4.42498 & 1.54101 \\
\hline 0 & 0.51775 & 3.70221 & -0.76268 \\
\hline $\mathrm{H}$ & 1.17745 & 5.10295 & 1.28703 \\
\hline $\mathrm{H}$ & -0.51555 & 5.00411 & 1.84848 \\
\hline $\mathrm{H}$ & 0.64868 & 3.79353 & 2.38767 \\
\hline $\mathrm{H}$ & -3.08254 & 3.22469 & -0.84535 \\
\hline $\mathrm{H}$ & -1.61906 & 3.31886 & -1.85483 \\
\hline $\mathrm{H}$ & -2.78075 & 2.00789 & -2.10156 \\
\hline $\mathrm{H}$ & -4.57565 & -1.72429 & -0.37938 \\
\hline $\mathrm{H}$ & -4.81196 & -1.95908 & 1.89318 \\
\hline
\end{tabular}

Conformer 3

Energy: -1308.92848 Hartree (Rel: $0.7 \mathrm{kcal} / \mathrm{mol}$ ) XYZ coordinates for conf 3 :

$\begin{array}{lrrr}C & 4.00928 & 2.45576 & 0.09385 \\ C & 2.78396 & 1.60810 & 0.49423 \\ C & 1.85346 & 1.27995 & -0.69292 \\ C & 0.61919 & 0.46505 & -0.23511 \\ C & -0.54384 & 0.56217 & -1.25333 \\ C & -1.89733 & 1.10285 & -0.69625 \\ \text { O } & 3.04148 & -0.84502 & 1.06509 \\ C & 3.25522 & 0.45010 & 1.38130 \\ C & 2.55393 & 0.56414 & -1.85667 \\ C & -2.54405 & 0.13996 & 0.32049 \\ O & 3.83590 & 0.67885 & 2.42524 \\ C & 3.13828 & -0.78940 & -1.40654 \\ C & 2.43145 & -1.37416 & -0.16864\end{array}$




\begin{tabular}{|c|c|c|c|}
\hline C & 0.92072 & -1.05794 & -0.10262 \\
\hline C & 0.07141 & -1.74511 & -1.20132 \\
\hline 0 & -0.74133 & -0.73434 & -1.81107 \\
\hline C & -3.25628 & -1.08073 & -0.31559 \\
\hline $\mathrm{C}$ & -3.05562 & -2.39171 & 0.46206 \\
\hline $\mathrm{C}$ & -1.59939 & -2.85521 & 0.54781 \\
\hline $\mathrm{H}$ & 3.68605 & 3.34492 & -0.45832 \\
\hline $\mathrm{H}$ & 4.54983 & 2.77709 & 0.98739 \\
\hline $\mathrm{H}$ & 4.70606 & 1.89727 & -0.54026 \\
\hline $\mathrm{H}$ & 2.19406 & 2.22069 & 1.19013 \\
\hline $\mathrm{H}$ & 1.48815 & 2.25071 & -1.05193 \\
\hline $\mathrm{H}$ & 0.29120 & 0.86119 & 0.72929 \\
\hline $\mathrm{H}$ & -0.25967 & 1.23552 & -2.07384 \\
\hline C & -2.81876 & 1.47149 & -1.86500 \\
\hline $\mathrm{H}$ & 1.82234 & 0.40553 & -2.65801 \\
\hline $\mathrm{H}$ & 3.33867 & 1.19419 & -2.28685 \\
\hline $\mathrm{H}$ & -1.76151 & -0.19396 & 1.01518 \\
\hline $\mathrm{H}$ & -3.26128 & 0.71333 & 0.91119 \\
\hline $\mathrm{H}$ & 4.20287 & -0.70366 & -1.15968 \\
\hline $\mathrm{H}$ & 3.07794 & -1.51491 & -2.22494 \\
\hline $\mathrm{C}$ & 2.72549 & -2.87063 & -0.03960 \\
\hline O & -1.45639 & 2.32936 & -0.00964 \\
\hline $\mathrm{H}$ & 0.57748 & -1.40511 & 0.87496 \\
\hline $\mathrm{H}$ & 0.71773 & -2.13743 & -1.99865 \\
\hline $\mathrm{H}$ & 2.30210 & -3.27118 & 0.88649 \\
\hline $\mathrm{H}$ & -4.33482 & -0.89635 & -0.36912 \\
\hline $\mathrm{H}$ & -2.90812 & -1.23999 & -1.33571 \\
\hline 0 & -3.69243 & -2.34039 & 1.73809 \\
\hline C & -1.08907 & -3.26478 & 1.71639 \\
\hline $\mathrm{C}$ & -0.78887 & -2.95666 & -0.73706 \\
\hline $\mathrm{H}$ & -0.07940 & -3.66076 & 1.79143 \\
\hline $\mathrm{H}$ & -1.67635 & -3.24674 & 2.62836 \\
\hline $\mathrm{H}$ & -0.09365 & -3.79719 & -0.63027 \\
\hline $\mathrm{H}$ & -1.44605 & -3.21142 & -1.57870 \\
\hline $\mathrm{H}$ & 2.31380 & -3.42734 & -0.88646 \\
\hline $\mathrm{H}$ & 3.80772 & -3.03475 & -0.01943 \\
\hline C & -2.32343 & 3.20149 & 0.54851 \\
\hline C & -1.57340 & 4.36831 & 1.15232 \\
\hline O & -3.53132 & 3.07243 & 0.56868 \\
\hline $\mathrm{H}$ & -2.28217 & 5.06340 & 1.60379 \\
\hline $\mathrm{H}$ & -0.86927 & 4.01229 & 1.91159 \\
\hline $\mathrm{H}$ & -0.99121 & 4.88199 & 0.38027 \\
\hline $\mathrm{H}$ & -3.81315 & 1.75642 & -1.51867 \\
\hline $\mathrm{H}$ & -2.38678 & 2.31042 & -2.42149 \\
\hline $\mathrm{H}$ & -2.90734 & 0.62534 & -2.55150 \\
\hline $\mathrm{H}$ & -3.60687 & -3.17379 & -0.07940 \\
\hline $\mathrm{H}$ & -3.27988 & -1.62092 & 2.24415 \\
\hline
\end{tabular}

Conformer 4

Energy: -1308.92896 Hartree (Rel: $0.4 \mathrm{kcal} / \mathrm{mol}$ )

XYZ coordinates for conf 4 :

$\begin{array}{rrrr}C & 4.51807 & 0.85624 & 0.11467 \\ \text { C } & 3.07473 & 0.51583 & 0.53937 \\ C & 2.05133 & 0.64120 & -0.60940 \\ \text { C } & 0.61994 & 0.29360 & -0.12623 \\ C & -0.46604 & 0.90638 & -1.04506 \\ C & -1.55352 & 1.77701 & -0.33425 \\ \text { O } & 2.41015 & -1.88912 & 0.96813 \\ C & 3.09167 & -0.78648 & 1.34820 \\ C & 2.40371 & -0.19174 & -1.84938 \\ C & -2.50084 & 0.93646 & 0.54719 \\ \text { O } & 3.72563 & -0.85442 & 2.38406 \\ C & 2.49396 & -1.68833 & -1.49864\end{array}$




\begin{tabular}{|c|c|c|c|}
\hline $\mathrm{C}$ & 1.63884 & -2.07316 & -0.27500 \\
\hline C & 0.34372 & -1.24150 & -0.13606 \\
\hline $\mathrm{C}$ & -0.68411 & -1.46621 & -1.27360 \\
\hline O & -1.11422 & -0.17494 & -1.72150 \\
\hline C & -3.59206 & 0.14265 & -0.20924 \\
\hline C & -3.86234 & -1.24668 & 0.39175 \\
\hline C & -2.64963 & -2.17876 & 0.37455 \\
\hline $\mathrm{H}$ & 4.54255 & 1.83299 & -0.38062 \\
\hline $\mathrm{H}$ & 5.16732 & 0.89357 & 0.99291 \\
\hline $\mathrm{H}$ & 4.92893 & 0.11409 & -0.57802 \\
\hline $\mathrm{H}$ & 2.78785 & 1.26266 & 1.29252 \\
\hline $\mathrm{H}$ & 2.05501 & 1.70019 & -0.89089 \\
\hline $\mathrm{H}$ & 0.49711 & 0.67919 & 0.89034 \\
\hline $\mathrm{H}$ & 0.00042 & 1.53526 & -1.80814 \\
\hline C & -2.32266 & 2.63470 & -1.34345 \\
\hline $\mathrm{H}$ & 1.62889 & -0.02985 & -2.60849 \\
\hline $\mathrm{H}$ & 3.34090 & 0.15213 & -2.29848 \\
\hline $\mathrm{H}$ & -1.88764 & 0.25773 & 1.15502 \\
\hline $\mathrm{H}$ & -2.96886 & 1.63086 & 1.25286 \\
\hline $\mathrm{H}$ & 3.52583 & -1.98427 & -1.27589 \\
\hline $\mathrm{H}$ & 2.18626 & -2.29692 & -2.35574 \\
\hline C & 1.38018 & -3.58109 & -0.25714 \\
\hline 0 & -0.92326 & 2.66876 & 0.66299 \\
\hline $\mathrm{H}$ & -0.11220 & -1.53069 & 0.81458 \\
\hline $\mathrm{H}$ & -0.19953 & -1.94481 & -2.13631 \\
\hline $\mathrm{H}$ & 0.86020 & -3.87634 & 0.65925 \\
\hline $\mathrm{H}$ & -4.53828 & 0.69418 & -0.19275 \\
\hline $\mathrm{H}$ & -3.31419 & 0.01180 & -1.25460 \\
\hline 0 & -4.45717 & -1.14233 & 1.68515 \\
\hline C & -2.32360 & -2.88374 & 1.46558 \\
\hline C & -1.90115 & -2.37564 & -0.93558 \\
\hline $\mathrm{H}$ & -1.50541 & -3.59934 & 1.45938 \\
\hline $\mathrm{H}$ & -2.88461 & -2.79147 & 2.38953 \\
\hline $\mathrm{H}$ & -1.52716 & -3.40533 & -0.95633 \\
\hline $\mathrm{H}$ & -2.59516 & -2.28973 & -1.78194 \\
\hline $\mathrm{H}$ & 0.78049 & -3.88699 & -1.11924 \\
\hline $\mathrm{H}$ & 2.33266 & -4.11941 & -0.29798 \\
\hline $\mathrm{C}$ & -0.00018 & 3.59424 & 0.33598 \\
\hline $\mathrm{C}$ & 0.33494 & 4.45038 & 1.53671 \\
\hline O & 0.49803 & 3.72766 & -0.76650 \\
\hline $\mathrm{H}$ & 1.15214 & 5.12775 & 1.28630 \\
\hline $\mathrm{H}$ & -0.54503 & 5.03119 & 1.83368 \\
\hline $\mathrm{H}$ & 0.61478 & 3.82304 & 2.38865 \\
\hline $\mathrm{H}$ & -3.10712 & 3.20793 & -0.83759 \\
\hline $\mathrm{H}$ & -1.64992 & 3.32699 & -1.85314 \\
\hline $\mathrm{H}$ & -2.79232 & 1.99833 & -2.09813 \\
\hline $\mathrm{H}$ & -4.64090 & -1.72050 & -0.22272 \\
\hline $\mathrm{H}$ & -3.81624 & -0.70141 & 2.26741 \\
\hline
\end{tabular}

Conformer 5

Energy: -1308.92959 Hartree (Rel: $0.0 \mathrm{kcal} / \mathrm{mol}$ ) XYZ coordinates for conf 5:

$\begin{array}{lrrr}\text { C } & 4.55699 & 0.77373 & -0.07111 \\ \text { C } & 3.14329 & 0.44524 & 0.45145 \\ \text { C } & 2.04522 & 0.55477 & -0.62871 \\ \text { C } & 0.65290 & 0.21552 & -0.03826 \\ C & -0.50952 & 0.83155 & -0.85650 \\ C & -1.47724 & 1.75056 & -0.05193 \\ \text { O } & 2.48800 & -1.94635 & 0.97056 \\ C & 3.20746 & -0.84491 & 1.27855 \\ C & 2.31832 & -0.30079 & -1.87336 \\ C & -2.18207 & 1.04028 & 1.13614 \\ \text { O } & 3.91345 & -0.90112 & 2.26730\end{array}$




\begin{tabular}{|c|c|c|}
\hline 2.43044 & -1.79087 & -1.50080 \\
\hline 1.64733 & -2.15450 & -0.22290 \\
\hline 0.36482 & -1.31644 & -0.02960 \\
\hline-0.71215 & -1.52925 & -1.12177 \\
\hline-1.27293 & -0.24275 & -1.40792 \\
\hline-3.56921 & 0.39633 & 0.94274 \\
\hline-3.70380 & -0.84252 & 0.03967 \\
\hline-2.73937 & -1.98207 & 0.38007 \\
\hline 4.55296 & 1.74403 & -0.57954 \\
\hline 5.26187 & 0.81933 & 0.76261 \\
\hline 4.91974 & 0.02134 & -0.77936 \\
\hline 2.90889 & 1.20383 & 1.21117 \\
\hline 2.03179 & 1.60821 & -0.93266 \\
\hline 0.61927 & 0.60120 & 0.98508 \\
\hline-0.11390 & 1.42531 & -1.68561 \\
\hline-2.46590 & 2.46302 & -0.97671 \\
\hline 1.49932 & -0.15078 & -2.58737 \\
\hline 3.22614 & 0.03333 & -2.38524 \\
\hline-1.50134 & 0.30341 & 1.57556 \\
\hline-2.30913 & 1.81760 & 1.89729 \\
\hline 3.47393 & -2.08241 & -1.33344 \\
\hline 2.07475 & -2.41454 & -2.32790 \\
\hline 1.38951 & -3.66121 & -0.16190 \\
\hline-0.66344 & 2.76683 & 0.66154 \\
\hline-0.05882 & -1.59895 & 0.93963 \\
\hline-0.25071 & -1.91216 & -2.04369 \\
\hline 0.89271 & -3.93368 & 0.77460 \\
\hline-4.28912 & 1.14015 & 0.58292 \\
\hline-3.91331 & 0.11031 & 1.94690 \\
\hline-5.06655 & -1.30172 & 0.05492 \\
\hline-2.70927 & -2.53300 & 1.60027 \\
\hline-1.86597 & -2.48696 & -0.74542 \\
\hline-2.07032 & -3.38237 & 1.82926 \\
\hline-3.32740 & -2.17106 & 2.42033 \\
\hline-1.44124 & -3.46429 & -0.49419 \\
\hline-2.48381 & -2.62685 & -1.64271 \\
\hline 0.76755 & -3.98772 & -1.00027 \\
\hline 2.34105 & -4.19996 & -0.21520 \\
\hline 0.11539 & 3.67286 & 0.03777 \\
\hline 0.69120 & 4.64556 & 1.04366 \\
\hline 0.33309 & 3.71447 & -1.15892 \\
\hline 1.40098 & 5.30768 & 0.54643 \\
\hline-0.11538 & 5.23946 & 1.48702 \\
\hline 1.18596 & 4.10741 & 1.85803 \\
\hline-3.12575 & 3.11973 & -0.39973 \\
\hline-1.93646 & 3.06151 & -1.72002 \\
\hline-3.08248 & 1.72910 & -1.50193 \\
\hline-3.53702 & -0.55129 & -0.99626 \\
\hline-5.22384 & -1.71322 & 0.92032 \\
\hline
\end{tabular}


Briarellin E - original (correct)

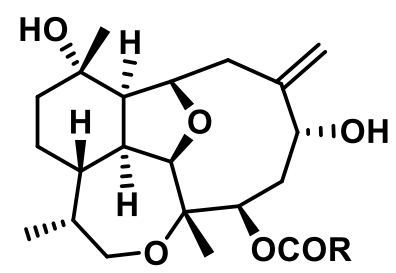

Rel energy (kcal/mol): $\begin{array}{cc}\text { Conf } 1 \text { Conf } 2 \\ 2.4 & 0.0\end{array}$

\begin{tabular}{|c|c|c|c|c|c|c|}
\hline C-nom & iGau & $\operatorname{Exp}$ & Calc & $\operatorname{diff}$ & 1 & 2 \\
\hline $\mathrm{C} 1-\mathrm{CH}$ & 4 & 39.43 & 40.85 & 1.42 & 39.88 & 40.87 \\
\hline $\mathrm{C} 2-\mathrm{CH}$ & 7 & 92.20 & 91.24 & -0.96 & 92.09 & 91.23 \\
\hline $\mathrm{C} 3-\mathrm{C}$ & 13 & 76.70 & 78.85 & 2.15 & 79.59 & 78.84 \\
\hline $\mathrm{C} 4-\mathrm{CH}^{\star} \star$ & 19 & 74.03 & 76.46 & 2.43 & 73.16 & 76.52 \\
\hline $\mathrm{C} 5-\mathrm{CH} 2 * \star \star *$ & 18 & 39.60 & 37.13 & -2.47 & 41.05 & 37.06 \\
\hline $\mathrm{C} 6-\mathrm{CH}^{*} *$ & 16 & 71.63 & 73.00 & 1.37 & 73.66 & 72.99 \\
\hline $\mathrm{C} 7-\mathrm{C}$ & 15 & 148.22 & 148.11 & -0.11 & [ 150.47 & 148.07 \\
\hline $\mathrm{C} 8-\mathrm{CH} 2$ & 14 & 38.98 & 40.42 & 1.44 & {$[\quad 38.94$} & 40.45 \\
\hline $\mathrm{C} 9-\mathrm{CH}$ & 9 & 81.96 & 82.38 & 0.42 & 83.80 & 82.36 \\
\hline $\mathrm{ClO}-\mathrm{CH}$ & 5 & 51.85 & 51.82 & -0.03 & 53.97 & 51.78 \\
\hline $\mathrm{C} 11-\mathrm{C}$ & 6 & 71.63 & 72.16 & 0.53 & 71.39 & 72.17 \\
\hline $\mathrm{C} 12-\mathrm{CH} 2 * * *$ & 1 & 37.06 & 35.61 & -1.45 & 36.46 & 35.59 \\
\hline $\mathrm{C} 13-\mathrm{CH} 2$ & 2 & 24.83 & 25.21 & 0.38 & 25.46 & 25.21 \\
\hline $\mathrm{Cl} 4-\mathrm{CH}$ & 3 & 38.85 & 39.87 & 1.02 & 40.37 & 39.86 \\
\hline $\mathrm{C} 15-\mathrm{CH}$ & 10 & 36.43 & 37.30 & 0.87 & 36.42 & 37.32 \\
\hline $\mathrm{C} 16-\mathrm{CH} 2$ & 11 & 67.39 & 67.42 & 0.03 & 68.46 & 67.40 \\
\hline $\mathrm{C} 17-\mathrm{CH} 3$ & 23 & 10.37 & 10.69 & 0.32 & 10.89 & 10.69 \\
\hline $\mathrm{C} 18-\mathrm{CH} 3$ & 22 & 17.89 & 17.89 & -0.00 & 17.17 & 17.90 \\
\hline $\mathrm{C} 19-\mathrm{CH} 2$ & 17 & 115.14 & 115.57 & 0.43 & {$[113.76$} & 115.60 \\
\hline $\mathrm{C} 20-\mathrm{CH} 3$ & 25 & 28.73 & 28.71 & -0.02 & [ 28.68 & 28.71 \\
\hline
\end{tabular}

13C chem shifts: $R M S D=1.19 p p m(M A E=0.89) \quad N=20 \quad\left\{\begin{array}{lll}-2.47 & 2.43\end{array}\right\}$

Fractions: 0.0170 .983

Note: chemical shifts for carbons C4/C6 and C5/C12 are swapped.

$\begin{array}{lrrr}\text { Conformer } & \\ \text { Energy: - } 1310.10116 & \text { Hartree (Rel: } & 2.4 & \mathrm{kcal} / \mathrm{mol}) \\ \text { XYZ coordinates for conf } 1: & & \\ \text { X } & & \\ \text { C } & -4.20635 & -0.50620 & -0.81326 \\ \text { C } & -3.26526 & -1.70174 & -1.10203 \\ \text { C } & -2.14558 & -1.79550 & -0.05516 \\ \text { C } & -1.26462 & -0.53835 & -0.16929 \\ \text { C } & -2.01744 & 0.74769 & -0.58933 \\ \text { C } & -3.51919 & 0.75600 & -0.20842 \\ \text { C } & -0.56325 & -0.16308 & 1.14697 \\ \text { O } & -0.52838 & 1.26760 & 1.20037 \\ \text { C } & -1.13496 & 1.84901 & 0.02963 \\ \text { C } & -1.29737 & -3.09025 & -0.08278 \\ \text { C } & -0.18786 & -3.04056 & 0.98958 \\ \text { O } & 0.85693 & -2.10735 & 0.68512 \\ \text { C } & 0.85312 & -0.80543 & 1.30175 \\ \text { C } & -0.07217 & 2.38761 & -0.95721 \\ \text { C } & 1.02247 & 3.19313 & -0.28196 \\ \text { C } & 2.37292 & 2.54153 & 0.01724 \\ \text { C } & 0.83086 & 4.48083 & 0.02564 \\ \text { C } & 2.34263 & 1.36376 & 1.02131\end{array}$




$\begin{array}{rrr}1.88712 & -0.00527 & 0.46388 \\ -2.00338 & 0.84420 & -1.68060 \\ -0.50379 & -0.72863 & -0.93270 \\ 1.26863 & -0.92505 & 2.77291 \\ -0.71349 & -3.45234 & -1.45758 \\ -2.63102 & -1.78360 & 0.93203 \\ -3.78732 & 0.86945 & 1.30175 \\ 3.06747 & 2.22792 & -1.19253 \\ 3.06808 & -0.85043 & 0.34657 \\ -3.89415 & 2.68192 & -0.38835 \\ -4.15479 & 1.87461 & -0.86148 \\ -5.00733 & -0.82557 & -0.13550 \\ -4.69239 & -0.19257 & -1.74285 \\ -2.82100 & -1.59208 & -2.09946 \\ -3.85310 & -2.62798 & -1.12661 \\ -1.16634 & -0.49714 & 2.00282 \\ -1.71396 & 2.70755 & 0.39125 \\ -1.96129 & -3.91422 & 0.22139 \\ 0.30212 & -4.02018 & 1.04339 \\ -0.62226 & -2.84220 & 1.97801 \\ -0.60238 & 3.01692 & -1.68293 \\ 0.35853 & 1.56284 & -1.53712 \\ 1.59756 & 5.07298 & 0.52034 \\ -0.10066 & 4.99495 & -0.20447 \\ 3.36115 & 1.22482 & 1.40037 \\ 1.72033 & 1.66458 & 1.86405 \\ 1.21563 & 0.04125 & 3.28405 \\ 2.28970 & -1.31012 & 2.84872 \\ 0.60187 & -1.61181 & 3.30440 \\ -0.17316 & -4.40486 & -1.39851 \\ -0.00424 & -2.69786 & -1.81223 \\ -1.49957 & -3.56449 & -2.21113 \\ -3.30085 & 1.75179 & 1.73425 \\ -3.43485 & -0.00421 & 1.85746 \\ -4.86661 & 0.95944 & 1.46419 \\ 1.47709 & 0.08624 & -0.54015 \\ 3.55137 & -1.09994 & -0.87862 \\ 4.66148 & -2.11774 & -0.83503 \\ 3.14120 & -0.56269 & -1.89736 \\ 5.42953 & -1.80717 & -0.11997 \\ 5.09820 & -2.23034 & -1.82766 \\ 4.26285 & -3.07884 & -0.49344 \\ 2.98260 & 3.31856 & 0.49286 \\ 2.85671 & 1.32318 & \end{array}$

Conformer 2

Energy: -1310.10498 Hartree (Rel: $0.0 \mathrm{kcal} / \mathrm{mol}$ ) XYZ coordinates for conf 2 :

$\begin{array}{lrrr}\text { C } & 4.05586 & 0.69544 & -1.05637 \\ C & 2.92726 & 1.67478 & -1.46758 \\ C & 1.88074 & 1.83094 & -0.35480 \\ C & 1.19162 & 0.46993 & -0.14373 \\ C & 2.13785 & -0.75405 & -0.25431 \\ C & 3.64167 & -0.41995 & -0.05352 \\ C & 0.54387 & 0.33347 & 1.24147 \\ \text { O } & 0.73610 & -1.01651 & 1.67260 \\ C & 1.54529 & -1.76765 & 0.75033 \\ C & 0.86828 & 2.98608 & -0.54821 \\ C & -0.14293 & 3.04470 & 0.61807 \\ \text { O } & -1.12370 & 2.00500 & 0.56820 \\ C & -0.95133 & 0.77522 & 1.30199 \\ C & 0.75676 & -2.95442 & 0.14481 \\ C & -0.45189 & -2.66481 & -0.73034\end{array}$




\begin{tabular}{|c|c|c|c|}
\hline $\mathrm{C}$ & -1.83047 & -2.70012 & -0.06229 \\
\hline C & -0.33531 & -2.49912 & -2.05342 \\
\hline C & -2.01876 & -1.63936 & 1.04888 \\
\hline $\mathrm{C}$ & -1.88964 & -0.19171 & 0.53174 \\
\hline $\mathrm{H}$ & 2.07883 & -1.16499 & -1.26631 \\
\hline $\mathrm{H}$ & 0.42479 & 0.34996 & -0.91445 \\
\hline C & -1.39102 & 0.96760 & 2.75861 \\
\hline $\mathrm{C}$ & 0.13979 & 2.99036 & -1.90104 \\
\hline $\mathrm{H}$ & 2.42470 & 2.08293 & 0.56620 \\
\hline $\mathrm{C}$ & 4.03057 & -0.04864 & 1.38842 \\
\hline O & -2.89913 & -2.67080 & -0.99558 \\
\hline 0 & -3.20501 & 0.44138 & 0.56146 \\
\hline $\mathrm{H}$ & 4.36493 & -2.22368 & 0.27728 \\
\hline 0 & 4.42211 & -1.57097 & -0.43981 \\
\hline $\mathrm{H}$ & 4.89424 & 1.25368 & -0.62328 \\
\hline $\mathrm{H}$ & 4.44632 & 0.19334 & -1.94759 \\
\hline $\mathrm{H}$ & 2.43240 & 1.30831 & -2.37584 \\
\hline $\mathrm{H}$ & 3.36449 & 2.64707 & -1.72697 \\
\hline $\mathrm{H}$ & 1.07944 & 0.97181 & 1.95896 \\
\hline $\mathrm{H}$ & 2.34128 & -2.23073 & 1.34908 \\
\hline $\mathrm{H}$ & 1.44558 & 3.92145 & -0.48112 \\
\hline $\mathrm{H}$ & -0.71429 & 3.97773 & 0.54467 \\
\hline $\mathrm{H}$ & 0.37783 & 3.06235 & 1.58510 \\
\hline $\mathrm{H}$ & 0.45122 & -3.59372 & 0.98418 \\
\hline $\mathrm{H}$ & 1.48506 & -3.53603 & -0.43455 \\
\hline $\mathrm{H}$ & -1.20588 & -2.34803 & -2.68300 \\
\hline $\mathrm{H}$ & 0.63028 & -2.54253 & -2.55121 \\
\hline $\mathrm{H}$ & -3.02192 & -1.77195 & 1.46725 \\
\hline $\mathrm{H}$ & -1.30146 & -1.82339 & 1.84722 \\
\hline $\mathrm{H}$ & -1.24474 & 0.04974 & 3.33778 \\
\hline $\mathrm{H}$ & -2.44436 & 1.25428 & 2.80637 \\
\hline $\mathrm{H}$ & -0.79471 & 1.75649 & 3.23028 \\
\hline $\mathrm{H}$ & -0.52624 & 3.85896 & -1.96957 \\
\hline $\mathrm{H}$ & -0.47688 & 2.09658 & -2.03462 \\
\hline $\mathrm{H}$ & 0.84557 & 3.04831 & -2.73579 \\
\hline $\mathrm{H}$ & 3.77165 & -0.84305 & 2.09838 \\
\hline $\mathrm{H}$ & 3.54772 & 0.86867 & 1.73523 \\
\hline $\mathrm{H}$ & 5.11436 & 0.10218 & 1.43472 \\
\hline $\mathrm{H}$ & -1.56661 & -0.19081 & -0.51025 \\
\hline C & -3.89591 & 0.54816 & -0.57918 \\
\hline C & -5.13488 & 1.38688 & -0.39538 \\
\hline 0 & -3.56370 & 0.02763 & -1.63528 \\
\hline $\mathrm{H}$ & -5.72722 & 1.00647 & 0.44254 \\
\hline $\mathrm{H}$ & -5.72865 & 1.37382 & -1.30973 \\
\hline $\mathrm{H}$ & -4.84732 & 2.41584 & -0.15455 \\
\hline $\mathrm{H}$ & -1.91343 & -3.68096 & 0.42840 \\
\hline $\mathrm{H}$ & -3.00484 & -1.75811 & -1.33229 \\
\hline
\end{tabular}

Briarellin F - original (correct)

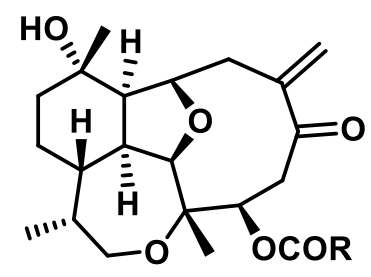

Note: C1-C9 fragment of briarellin $F$ matches that of asbestinin-19 also correctly assigned) with $\mathrm{rmsd}(\exp -\mathrm{exp})=0.65 \mathrm{ppm}$ 


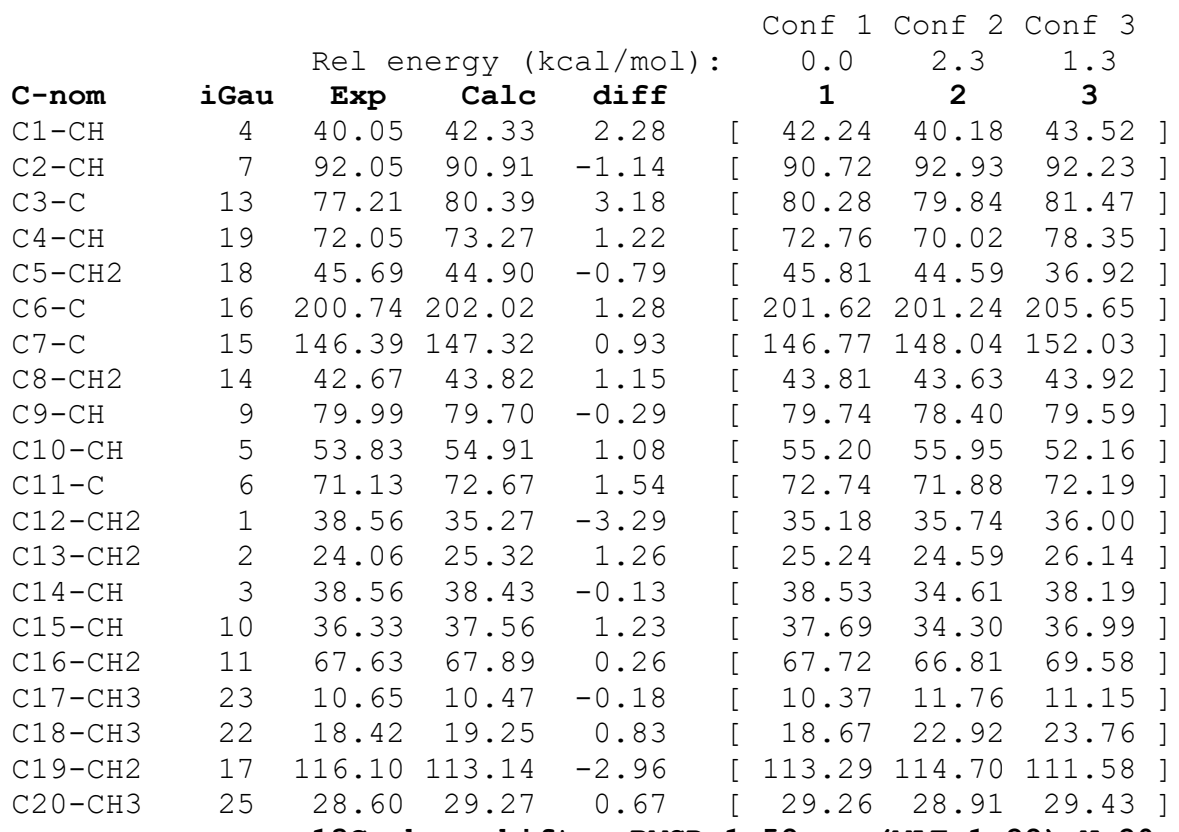

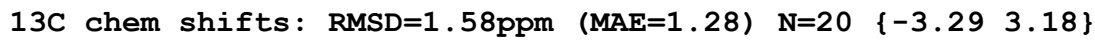

Fractions: $0.8830 .017 \quad 0.100$

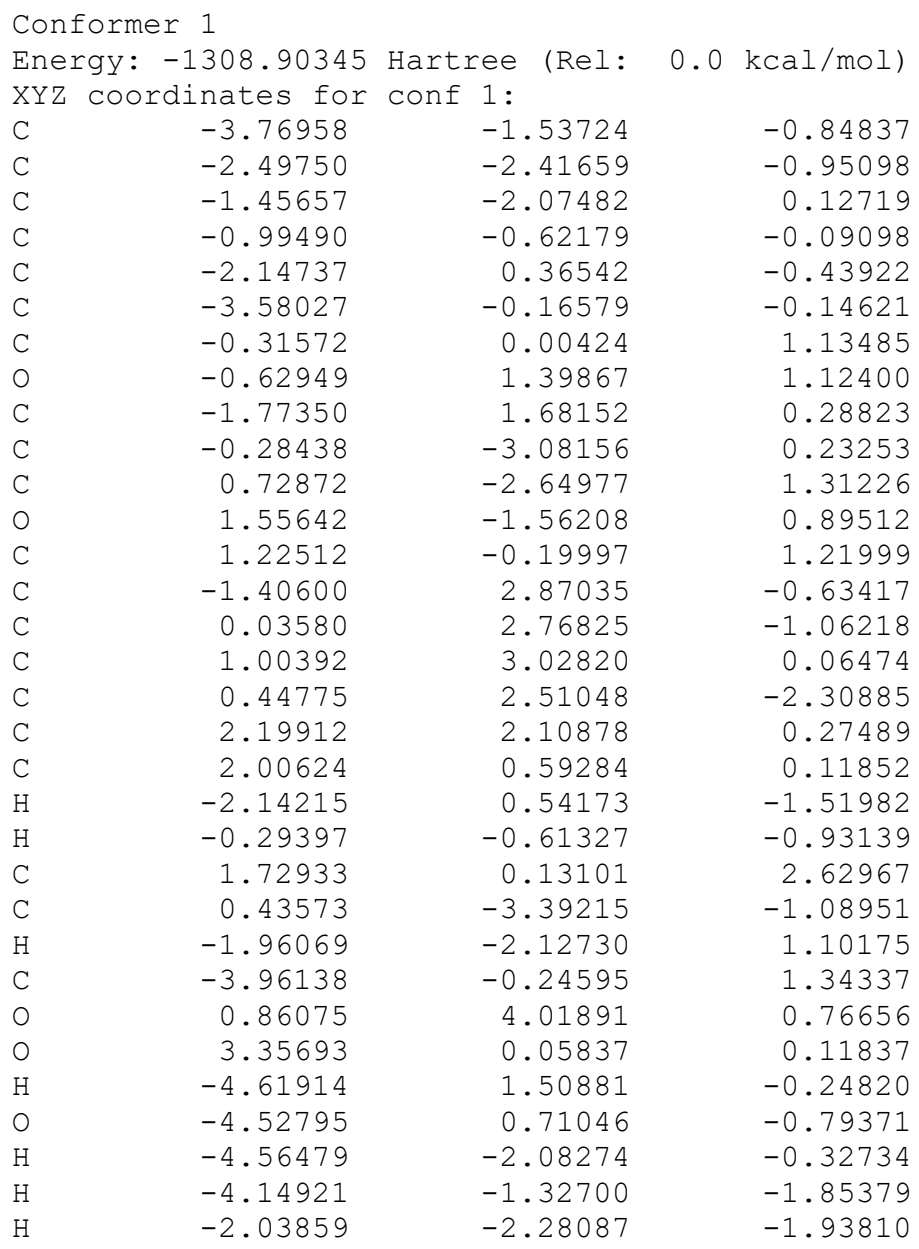




$\begin{array}{lrrr}\mathrm{H} & -2.77717 & -3.47544 & -0.88753 \\ \mathrm{H} & -0.75026 & -0.42665 & 2.05012 \\ \mathrm{H} & -2.58686 & 2.00596 & 0.94754 \\ \mathrm{H} & -0.72148 & -4.02335 & 0.59971 \\ \mathrm{H} & 1.42273 & -3.47730 & 1.50151 \\ \mathrm{H} & 0.22315 & -2.43115 & 2.26270 \\ \mathrm{H} & -1.52785 & 3.80488 & -0.07563 \\ \mathrm{H} & -2.08547 & 2.89689 & -1.49193 \\ \mathrm{H} & 1.50062 & 2.43337 & -2.56822 \\ \mathrm{H} & -0.25972 & 2.39143 & -3.12585 \\ \mathrm{H} & 2.94747 & 2.39276 & -0.47935 \\ \mathrm{H} & 2.63232 & 2.35792 & 1.24703 \\ \mathrm{H} & 1.43287 & 1.14129 & 2.93045 \\ \mathrm{H} & 2.81903 & 0.04926 & 2.67453 \\ \mathrm{H} & 1.30128 & -0.57127 & 3.35357 \\ \mathrm{H} & 1.20567 & -4.15607 & -0.92498 \\ \mathrm{H} & 0.93817 & -2.51195 & -1.50061 \\ \mathrm{H} & -0.25729 & -3.78193 & -1.84174 \\ \mathrm{H} & -3.91673 & 0.73359 & 1.83387 \\ \mathrm{H} & -3.32129 & -0.92391 & 1.91357 \\ \mathrm{H} & -4.99198 & -0.60713 & 1.42329 \\ \mathrm{H} & 1.56846 & 0.36716 & -0.85252 \\ \mathrm{C} & 3.75100 & -0.68424 & -0.94027 \\ \mathrm{C} & 5.11057 & -1.28749 & -0.68385 \\ \mathrm{O} & 3.10052 & -0.83682 & -1.95500 \\ \mathrm{H} & 5.79431 & -0.54144 & -0.26909 \\ \mathrm{H} & 5.51362 & -1.69755 & -1.61068 \\ \mathrm{H} & 5.01083 & -2.09104 & 0.05457\end{array}$

Conformer 2

Energy: -1308.89971 Hartree (Rel: $2.3 \mathrm{kcal} / \mathrm{mol}$ ) XYZ coordinates for conf 2 :

$\begin{array}{lrrr}\mathrm{C} & -4.00005 & -1.37103 & -0.42103 \\ \mathrm{C} & -2.79398 & -2.26812 & -0.78098 \\ \mathrm{C} & -1.55489 & -1.98089 & 0.08746 \\ \mathrm{C} & -1.10928 & -0.52218 & -0.14091 \\ \mathrm{C} & -2.25320 & 0.46598 & -0.48605 \\ \mathrm{C} & -3.64381 & 0.05688 & 0.07618 \\ \mathrm{C} & -0.46565 & 0.11897 & 1.09803 \\ \mathrm{O} & -0.68974 & 1.53075 & 0.99237 \\ \mathrm{C} & -1.72135 & 1.82613 & 0.02267 \\ \mathrm{C} & -0.39870 & -3.01066 & -0.09888 \\ \mathrm{C} & 1.00088 & -2.46631 & 0.28166 \\ \mathrm{O} & 1.08249 & -1.66087 & 1.45959 \\ \mathrm{C} & 1.02228 & -0.22260 & 1.35937 \\ \mathrm{C} & -1.12818 & 2.76931 & -1.05911 \\ \mathrm{C} & 0.31741 & 2.43508 & -1.32830 \\ \mathrm{C} & 1.23265 & 2.78336 & -0.18223 \\ \mathrm{C} & 0.78551 & 1.92551 & -2.47449 \\ \mathrm{C} & 2.32529 & 1.81217 & 0.24773 \\ \mathrm{C} & 2.00554 & 0.30645 & 0.26993 \\ \mathrm{H} & -2.39075 & 0.50782 & -1.57145 \\ \mathrm{H} & -0.40585 & -0.48846 & -0.98001 \\ \mathrm{C} & 1.43045 & 0.26820 & 2.75180 \\ \mathrm{C} & -0.26947 & -3.57918 & -1.52405 \\ \mathrm{H} & -1.86611 & -2.06728 & 1.13657 \\ \mathrm{C} & -3.77469 & 0.14270 & 1.60682 \\ \mathrm{O} & 1.14067 & 3.87694 & 0.35576 \\ \mathrm{O} & 3.26440 & -0.37784 & 0.52239 \\ \mathrm{H} & -4.60175 & 1.77604 & -0.04921 \\ \mathrm{O} & -4.64115 & 0.92074 & -0.50788 \\ \mathrm{H} & -4.61351 & -1.86244 & 0.34348 \\ \mathrm{H} & -4.63868 & -1.25596 & -1.30304 \\ & & & \end{array}$




$\begin{array}{rrrr}\mathrm{H} & -2.52954 & -2.11890 & -1.83512 \\ \mathrm{H} & -3.09159 & -3.31953 & -0.68752 \\ \mathrm{H} & -0.98957 & -0.24347 & 1.99323 \\ \mathrm{H} & -2.50956 & 2.37960 & 0.54667 \\ \mathrm{H} & -0.62293 & -3.85186 & 0.57071 \\ \mathrm{H} & 1.42234 & -1.94190 & -0.58185 \\ \mathrm{H} & 1.65915 & -3.32187 & 0.47256 \\ \mathrm{H} & -1.16956 & 3.79821 & -0.68529 \\ \mathrm{H} & -1.73080 & 2.71901 & -1.97157 \\ \mathrm{H} & 1.83535 & 1.68204 & -2.61694 \\ \mathrm{H} & 0.12850 & 1.74715 & -3.32244 \\ \mathrm{H} & 3.14531 & 1.93383 & -0.47389 \\ \mathrm{H} & 2.70194 & 2.15336 & 1.21467 \\ \mathrm{H} & 1.23251 & 1.33805 & 2.86191 \\ \mathrm{H} & 2.49161 & 0.07646 & 2.93307 \\ \mathrm{H} & 0.84796 & -0.27012 & 3.50558 \\ \mathrm{H} & 0.59771 & -4.24769 & -1.59035 \\ \mathrm{H} & -0.12537 & -2.78301 & -2.26555 \\ \mathrm{H} & -1.15078 & -4.15670 & -1.81654 \\ \mathrm{H} & -3.49768 & 1.12952 & 1.99638 \\ \mathrm{H} & -3.15963 & -0.60091 & 2.12068 \\ \mathrm{H} & -4.81947 & -0.04459 & 1.87684 \\ \mathrm{H} & 1.67162 & 0.01654 & -0.72451 \\ \mathrm{C} & 4.03975 & -0.66891 & -0.54538 \\ \mathrm{C} & 5.31014 & -1.36020 & -0.11564 \\ \mathrm{O} & 3.73985 & -0.39814 & -1.69293 \\ \mathrm{H} & 5.89854 & -0.69201 & 0.52216 \\ \mathrm{H} & 5.89305 & -1.63538 & -0.99517 \\ \mathrm{H} & 5.07543 & -2.25201 & 0.47371\end{array}$

Conformer 3

Energy: -1308.90140 Hartree (Rel: $1.3 \mathrm{kcal} / \mathrm{mol}$ ) XYZ coordinates for conf 3 :

$\begin{array}{lrrr}C & -4.28441 & -0.31514 & -0.28811 \\ \mathrm{C} & -3.75400 & 0.89270 & 0.51976 \\ \mathrm{C} & -2.41466 & 1.41671 & -0.02591 \\ \mathrm{C} & -1.35155 & 0.31313 & 0.13695 \\ \mathrm{C} & -1.90685 & -1.12729 & -0.00585 \\ \mathrm{C} & -3.18647 & -1.24714 & -0.87525 \\ \mathrm{C} & -0.19895 & 0.34267 & -0.87667 \\ \mathrm{O} & 0.32950 & -0.98917 & -0.88880 \\ \mathrm{C} & -0.68190 & -1.93980 & -0.48756 \\ \mathrm{C} & -1.96572 & 2.76635 & 0.58556 \\ \mathrm{C} & -0.64628 & 3.23904 & -0.04639 \\ \mathrm{O} & 0.47866 & 2.42109 & 0.29559 \\ \mathrm{C} & 0.93003 & 1.39860 & -0.61297 \\ \mathrm{C} & -0.06935 & -2.85833 & 0.59088 \\ \mathrm{C} & 0.54938 & -2.03526 & 1.69245 \\ \mathrm{C} & 1.85648 & -1.38301 & 1.31908 \\ \mathrm{C} & 0.04173 & -1.90372 & 2.92343 \\ \mathrm{C} & 2.03383 & 0.12358 & 1.46680 \\ \mathrm{C} & 2.20337 & 0.89068 & 0.12885 \\ \mathrm{H} & -2.20974 & -1.49184 & 0.98121 \\ \mathrm{H} & -0.92443 & 0.38850 & 1.14014 \\ \mathrm{C} & 1.38047 & 2.01125 & -1.95052 \\ \mathrm{C} & -1.88525 & 2.79079 & 2.12027 \\ \mathrm{H} & -2.55489 & 1.60907 & -1.10050 \\ \mathrm{C} & -2.98280 & -0.97584 & -2.37552 \\ \mathrm{O} & 2.78005 & -2.08448 & 0.93002 \\ \mathrm{O} & 2.99718 & 0.13082 & -0.80011 \\ \mathrm{H} & -3.16044 & -3.17975 & -1.26724 \\ \mathrm{O} & -3.71014 & -2.58482 & -0.73104 \\ \mathrm{H} & -4.92054 & 0.03689 & -1.10903\end{array}$




$\begin{array}{rrr}-4.92108 & -0.93185 & 0.35472 \\ -3.62288 & 0.60271 & 1.56974 \\ -4.50576 & 1.69189 & 0.51495 \\ -0.61070 & 0.55508 & -1.87492 \\ -0.92815 & -2.56045 & -1.35973 \\ -2.71596 & 3.51445 & 0.28501 \\ -0.40216 & 4.24025 & 0.32834 \\ -0.76071 & 3.31958 & -1.13377 \\ 0.71123 & -3.47412 & 0.12967 \\ -0.84772 & -3.52621 & 0.97601 \\ 0.51352 & -1.27928 & 3.67858 \\ -0.85038 & -2.44410 & 3.23098 \\ 1.21867 & 0.58448 & 2.02809 \\ 2.96337 & 0.27448 & 2.02531 \\ 1.75140 & 1.22714 & -2.61514 \\ 2.17809 & 2.74448 & -1.79210 \\ 0.54952 & 2.50922 & -2.45829 \\ -1.60982 & 3.79418 & 2.46625 \\ -1.13028 & 2.09655 & 2.50255 \\ -2.84563 & 2.53612 & 2.57961 \\ -2.20629 & -1.61623 & -2.81078 \\ -2.70722 & 0.06224 & -2.58108 \\ -3.92161 & -1.18375 & -2.89990 \\ 2.75303 & 1.80390 & 0.36912 \\ 4.33436 & 0.11123 & -0.59726 \\ 5.00746 & -0.82857 & -1.56416 \\ 4.89625 & 0.75889 & 0.26340 \\ 4.79277 & -1.85613 & -1.25067 \\ 6.08556 & -0.66308 & -1.55082 \\ 4.61366 & -0.69880 & -2.57602\end{array}$


Briarellin H - original

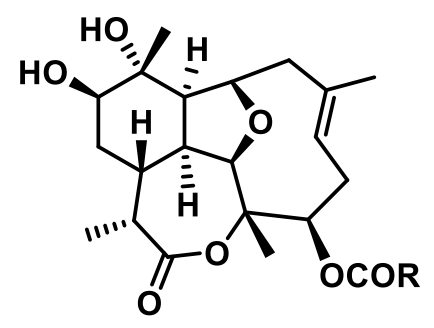

Rel energy (kcal/mol): $\begin{array}{ccc}\text { Conf } 1 & \text { Conf } 2 \\ 0.0 & 3.3\end{array}$

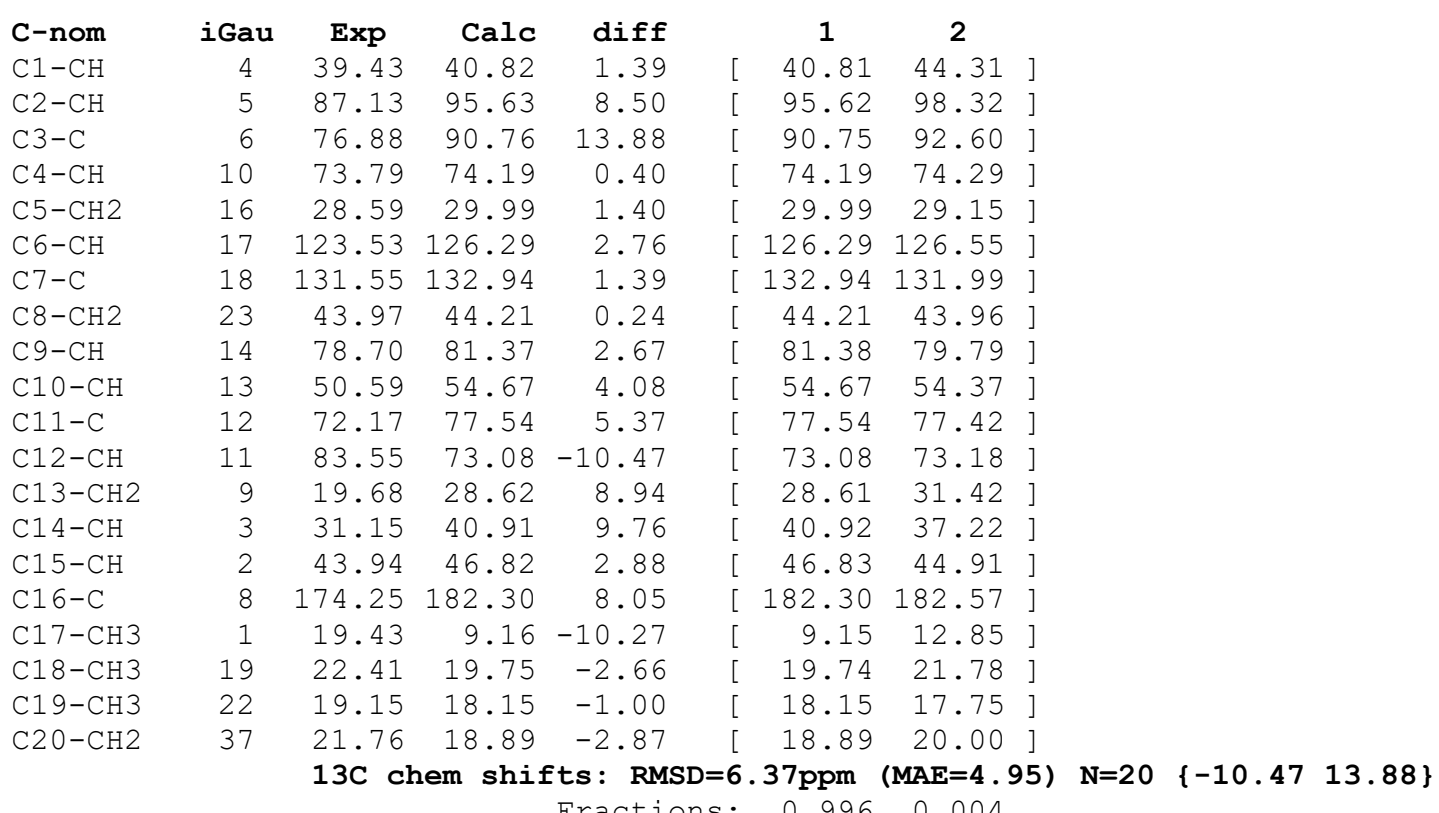

Fractions: 0.9960 .004

Conformer 1

Energy: -1423.43227 Hartree (Rel: $0.0 \mathrm{kcal} / \mathrm{mol}$ )

XYZ coordinates for conf 1 :

$\begin{array}{lrrr}\text { C } & 0.69948 & -2.98379 & -0.92898 \\ \text { C } & -0.08990 & -2.73149 & 0.36813 \\ \text { C } & -1.35006 & -1.80465 & 0.22838 \\ \text { C } & -0.98023 & -0.31467 & 0.00377 \\ \text { C } & -0.36812 & 0.30049 & 1.28819 \\ \text { C } & 1.20753 & 0.32276 & 1.31069 \\ \text { O } & 1.54374 & -1.07401 & 0.98677 \\ \text { C } & 0.79578 & -2.12884 & 1.45278 \\ \text { C } & -2.37545 & -2.35218 & -0.78756 \\ \text { C } & 1.97483 & 1.07207 & 0.16867 \\ \text { C } & -3.41959 & -1.31899 & -1.25490 \\ \text { C } & -3.56003 & -0.07904 & -0.33896 \\ \text { C } & -2.17844 & 0.62177 & -0.34367 \\ \text { C } & -2.03409 & 1.84978 & 0.59000 \\ \text { O } & -1.03469 & 1.52252 & 1.56746 \\ \text { C } & 2.08221 & 2.61075 & 0.22198 \\ \text { C } & 0.70524 & 3.19798 & 0.32190 \\ \text { C } & -0.24915 & 3.15237 & -0.62304\end{array}$




\begin{tabular}{|c|c|c|}
\hline 1.73375 & 0.68890 & 2.69631 \\
\hline-4.66703 & -1.98212 & -1.41724 \\
\hline-4.50114 & 0.74479 & -1.06721 \\
\hline 0.00091 & 2.87947 & -2.08690 \\
\hline-1.67463 & 3.19341 & -0.12371 \\
\hline 3.33764 & 0.57427 & 0.19627 \\
\hline 0.85940 & -2.51976 & 2.59686 \\
\hline 0.08391 & -3.51440 & -1.66105 \\
\hline 1.57087 & -3.61485 & -0.72108 \\
\hline 1.06512 & -2.05852 & -1.38004 \\
\hline-0.44384 & -3.69122 & 0.76055 \\
\hline-1.83067 & -1.85739 & 1.21119 \\
\hline-0.25983 & -0.25917 & -0.81685 \\
\hline-0.60655 & -0.35583 & 2.13851 \\
\hline-1.87580 & -2.75403 & -1.67344 \\
\hline-2.91331 & -3.18859 & -0.32713 \\
\hline 1.54150 & 0.77874 & -0.78656 \\
\hline-3.10153 & -0.91171 & -2.22827 \\
\hline-4.17058 & -0.40485 & 1.02974 \\
\hline-2.06828 & 0.95516 & -1.37881 \\
\hline-2.96261 & 2.02620 & 1.14638 \\
\hline 2.70317 & 2.90718 & 1.07366 \\
\hline 2.61932 & 2.91478 & -0.68457 \\
\hline 0.38968 & 3.45078 & 1.32980 \\
\hline 2.82532 & 0.63181 & 2.71640 \\
\hline 1.33558 & 0.00006 & 3.44592 \\
\hline 1.42517 & 1.70546 & 2.96150 \\
\hline-5.29514 & -1.27781 & -1.65885 \\
\hline-4.47713 & 0.51007 & 1.55006 \\
\hline-3.48626 & -0.93577 & 1.69413 \\
\hline-4.90474 & 1.37323 & -0.44801 \\
\hline-0.48267 & 3.65120 & -2.70123 \\
\hline-0.41839 & 1.91983 & -2.41710 \\
\hline 1.06652 & 2.87655 & -2.33427 \\
\hline-2.40762 & 3.36495 & -0.92167 \\
\hline-1.80023 & 3.99604 & 0.61293 \\
\hline 3.78748 & -0.13069 & -0.86726 \\
\hline 5.19246 & -0.63751 & -0.61113 \\
\hline 3.14878 & -0.32139 & -1.88359 \\
\hline 5.83132 & -1.28284 & -1.83946 \\
\hline 5.79281 & 0.20043 & -0.23711 \\
\hline 5.12931 & -1.35019 & 0.22201 \\
\hline 6.83171 & -1.65401 & -1.59511 \\
\hline 5.23005 & -2.12298 & -2.19916 \\
\hline 5.92397 & -0.56439 & -2.66025 \\
\hline-5.05744 & -1.02710 & 0.88336 \\
\hline
\end{tabular}

Conformer 2

Energy: -1423.42696 Hartree (Rel: $3.3 \mathrm{kcal} / \mathrm{mol}$ ) XYZ coordinates for conf 2 :

$\begin{array}{lrrr}\text { C } & 0.16143 & 3.63278 & -1.01871 \\ C & 0.27825 & 2.71169 & 0.20488 \\ C & 1.51449 & 1.72631 & 0.22964 \\ C & 1.14045 & 0.24404 & -0.05600 \\ C & 0.56975 & -0.46649 & 1.19616 \\ C & -0.98323 & -0.32050 & 1.39689 \\ \text { O } & -1.07331 & 1.15183 & 1.50853 \\ C & -1.04213 & 1.97212 & 0.40438 \\ C & 2.66156 & 2.20651 & -0.68347 \\ C & -1.98426 & -0.79872 & 0.29358 \\ C & 3.87955 & 1.25870 & -0.78161 \\ C & 3.71604 & -0.09923 & -0.06080 \\ C & 2.33237 & -0.66512 & -0.47245\end{array}$




\begin{tabular}{|c|c|c|}
\hline 2.00746 & -2.04146 & 0.14817 \\
\hline 1.11545 & -1.78385 & 1.24287 \\
\hline-2.19833 & -2.31668 & 0.08798 \\
\hline-0.88412 & -3.00809 & -0.11592 \\
\hline-0.02622 & -2.82622 & -1.13360 \\
\hline-1.38305 & -0.84553 & 2.77271 \\
\hline 5.03731 & 1.95938 & -0.33293 \\
\hline 4.75533 & -0.90833 & -0.65745 \\
\hline-0.36921 & -2.19139 & -2.45885 \\
\hline 1.42083 & -3.12203 & -0.82142 \\
\hline-3.26297 & -0.22836 & 0.66761 \\
\hline-2.01248 & 2.14474 & -0.29847 \\
\hline 1.03912 & 4.27704 & -1.11195 \\
\hline-0.71943 & 4.27144 & -0.91562 \\
\hline 0.04464 & 3.06555 & -1.94788 \\
\hline 0.38269 & 3.35095 & 1.09327 \\
\hline 1.88767 & 1.76116 & 1.25568 \\
\hline 0.40830 & 0.21550 & -0.87160 \\
\hline 0.95117 & 0.05016 & 2.08686 \\
\hline 2.27847 & 2.36942 & -1.69471 \\
\hline 3.03143 & 3.17506 & -0.33076 \\
\hline-1.70388 & -0.36451 & -0.66636 \\
\hline 4.01893 & 0.98962 & -1.83944 \\
\hline 3.97973 & -0.03198 & 1.44976 \\
\hline 2.37330 & -0.75097 & -1.56353 \\
\hline 2.91163 & -2.48356 & 0.58401 \\
\hline-2.72108 & -2.72489 & 0.95898 \\
\hline-2.87660 & -2.41070 & -0.76677 \\
\hline-0.50095 & -3.52160 & 0.76108 \\
\hline-2.44501 & -0.67149 & 2.95958 \\
\hline-0.80087 & -0.34066 & 3.54931 \\
\hline-1.17817 & -1.91860 & 2.83506 \\
\hline 5.78256 & 1.34747 & -0.46175 \\
\hline 3.92967 & -1.03111 & 1.89791 \\
\hline 3.26942 & 0.59816 & 1.98680 \\
\hline 4.93680 & -1.65650 & -0.06686 \\
\hline-0.13198 & -2.88250 & -3.27989 \\
\hline 0.21704 & -1.28245 & -2.65064 \\
\hline-1.42813 & -1.92919 & -2.53897 \\
\hline 2.05787 & -3.17107 & -1.71302 \\
\hline 1.50679 & -4.08874 & -0.31120 \\
\hline-4.10030 & 0.11862 & -0.33527 \\
\hline-5.29790 & 0.87090 & 0.20513 \\
\hline-3.90063 & -0.14000 & -1.50589 \\
\hline-6.41141 & 1.04842 & -0.82549 \\
\hline-5.65520 & 0.35463 & 1.10377 \\
\hline-4.91929 & 1.84317 & 0.54730 \\
\hline-7.23331 & 1.63327 & -0.40006 \\
\hline-6.04140 & 1.56801 & -1.71408 \\
\hline-6.81148 & 0.08119 & -1.14712 \\
\hline 4.97921 & 0.37346 & 1.62435 \\
\hline
\end{tabular}




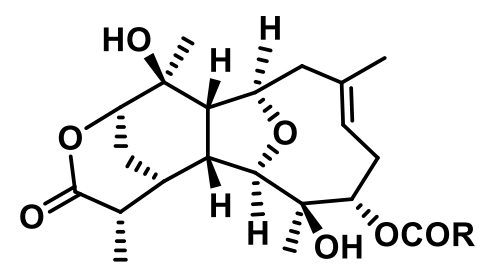

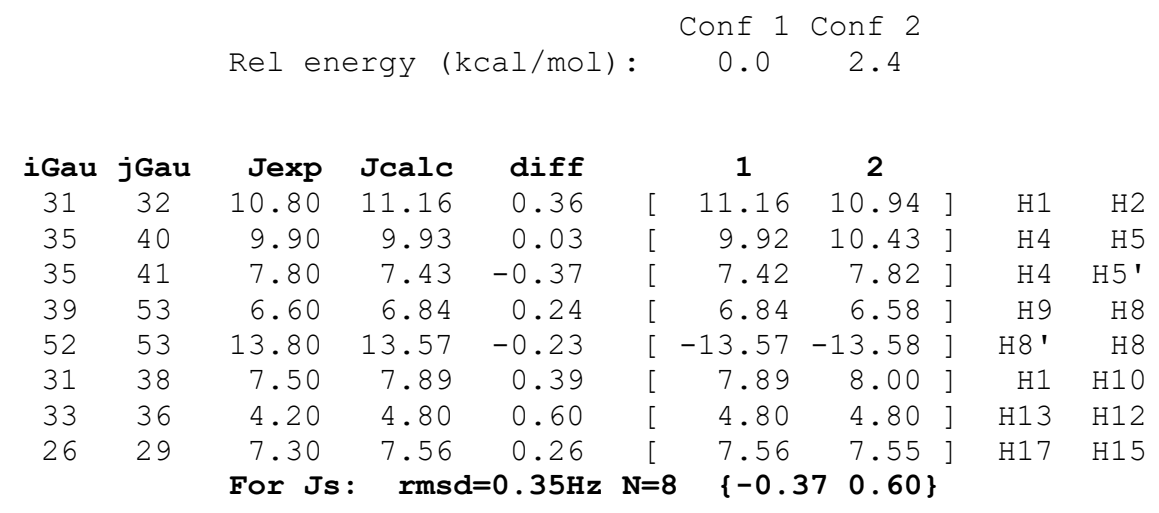

\begin{tabular}{|c|c|c|c|c|c|c|c|}
\hline H-nom & iGau & $\operatorname{Exp}$ & Calc & $\operatorname{diff}$ & 1 & 2 & \\
\hline $\mathrm{H} 1$ & 31 & 2.28 & 2.28 & 0.00 & 2.28 & $2.36]$ & \\
\hline $\mathrm{H} 2$ & 32 & 3.89 & 4.10 & 0.21 & 4.11 & $3.79]$ & \\
\hline $\mathrm{H} 4$ & 35 & 5.01 & 5.00 & -0.01 & 4.99 & $5.48]$ & \\
\hline H5 & 40 & 1.97 & 2.15 & 0.18 & 2.15 & $2.42]$ & \\
\hline H5 ' & 41 & 2.96 & 3.13 & 0.17 & 3.14 & $2.83]$ & \\
\hline H 6 & 42 & 5.43 & 5.86 & 0.43 & 5.86 & $5.87]$ & \\
\hline H8 & 53 & 2.51 & 2.69 & 0.18 & 2.69 & $2.66]$ & \\
\hline H8' & 52 & 1.88 & 2.04 & 0.16 & 2.04 & $2.04]$ & \\
\hline H9 & 39 & 4.48 & 4.52 & 0.04 & 4.52 & $4.53]$ & \\
\hline $\mathrm{H} 10$ & 38 & 2.01 & 2.08 & 0.07 & 2.08 & $2.09]$ & \\
\hline H12 & 36 & 4.31 & 4.39 & 0.08 & 4.39 & $4.38]$ & \\
\hline H13 & 33 & 2.13 & 2.14 & 0.01 & 2.14 & $2.14]$ & \\
\hline $\mathrm{H} 13^{\prime}$ & 34 & 2.06 & 1.93 & -0.13 & 1.93 & $1.84]$ & \\
\hline H1 4 & 30 & 2.29 & 2.34 & 0.05 & 2.34 & $2.46]$ & \\
\hline $\mathrm{H} 15$ & 29 & 2.56 & 2.56 & 0.00 & 2.56 & $2.64]$ & \\
\hline $\mathrm{H} 17$ & 26 & 1.37 & 1.43 & 0.06 & 1.43 & $1.50]$ & \\
\hline H1 8 & 45 & 1.46 & 1.61 & 0.15 & 1.61 & $1.58]$ & \\
\hline $\mathrm{H} 19$ & 49 & 1.81 & 1.95 & 0.14 & 1.95 & $1.92]$ & \\
\hline $\mathrm{H} 2 \mathrm{O}$ & 47 & 1.25 & 1.38 & 0.13 & {$\left[\begin{array}{ll}1.38 \\
\end{array}\right.$} & $\left.\begin{array}{lll}8 & 1.35\end{array}\right]$ & \\
\hline & & $\begin{array}{l}1 \mathrm{H} \text { chem } \\
\mathrm{m}=1.000\end{array}$ & $\begin{array}{c}\text { shifts } \\
b=0.0\end{array}$ & $\begin{array}{l}: \text { RMSD }=0 \\
0\end{array}$ & $.15 \mathrm{ppm}$ & $(\mathrm{MAE}=0.12)$ & 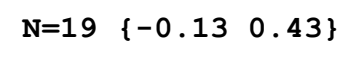 \\
\hline
\end{tabular}

\begin{tabular}{|c|c|c|c|c|c|c|}
\hline C-nom & iGau & $\operatorname{Exp}$ & Calc & $\operatorname{diff}$ & 1 & 2 \\
\hline $\mathrm{Cl}-\mathrm{CH}$ & 4 & 45.25 & 46.87 & 1.62 & 46.87 & 47.10 \\
\hline $\mathrm{C} 2-\mathrm{CH}$ & 5 & 87.13 & 87.23 & 0.10 & 87.18 & 89.92 \\
\hline C3-C & 6 & 76.88 & 78.09 & 1.21 & 78.07 & 79.02 \\
\hline $\mathrm{C} 4-\mathrm{CH}$ & 10 & 73.79 & 78.23 & 4.44 & 78.34 & 71.94 \\
\hline $\mathrm{C} 5-\mathrm{CH} 2$ & 16 & 28.59 & 28.88 & 0.29 & 28.86 & 29.78 \\
\hline $\mathrm{C} 6-\mathrm{CH}$ & 17 & 123.53 & 125.07 & 1.54 & {$[125.07$} & 125.12 \\
\hline $\mathrm{C} 7-\mathrm{C}$ & 18 & 131.55 & 134.06 & 2.51 & {$[134.07$} & 133.75 \\
\hline $\mathrm{C} 8-\mathrm{CH} 2$ & 23 & 43.97 & 43.24 & -0.73 & {$[\quad 43.24$} & 43.38 \\
\hline $\mathrm{C} 9-\mathrm{CH}$ & 14 & 78.70 & 79.80 & 1.10 & 79.79 & 80.19 \\
\hline $\mathrm{Cl} 0-\mathrm{CH}$ & 13 & 50.59 & 51.33 & 0.74 & 51.34 & 50.99 \\
\hline $\mathrm{C} 11-\mathrm{C}$ & 12 & 72.17 & 73.42 & 1.25 & 73.42 & 73.33 \\
\hline
\end{tabular}




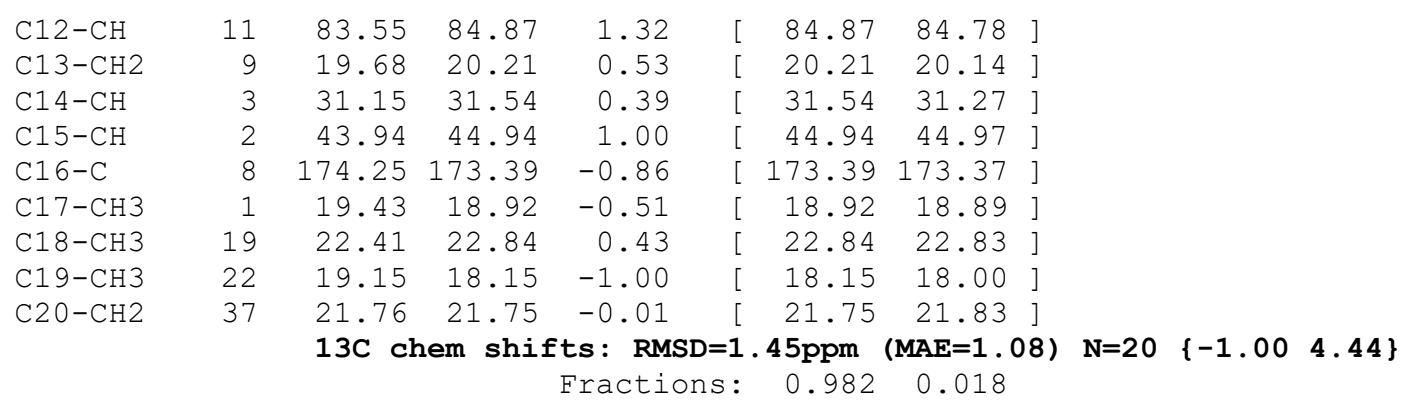

NOTICE :

C1: chemical shift of 39.43 ppm is corrected to 45.25 ppm (a typo in the original publication)

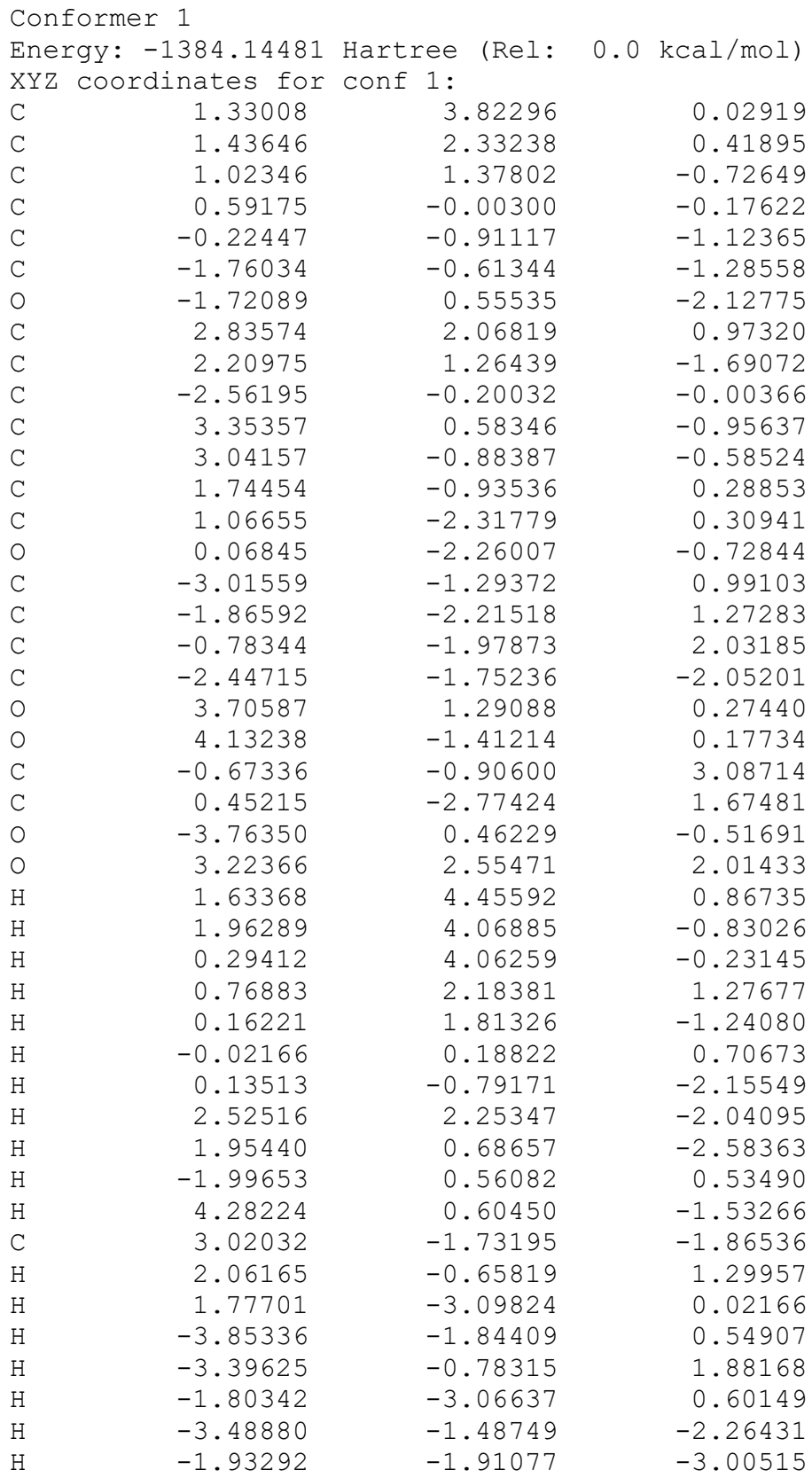




$\begin{array}{rrrr}\mathrm{H} & -2.43313 & -2.69210 & -1.49570 \\ \mathrm{H} & 4.35078 & -0.74384 & 0.85037 \\ \mathrm{H} & 3.06375 & -2.79309 & -1.60905 \\ \mathrm{H} & 2.13078 & -1.56697 & -2.47460 \\ \mathrm{H} & -0.46890 & -1.36362 & 4.06529 \\ \mathrm{H} & 0.15977 & -0.21659 & 2.89720 \\ \mathrm{H} & -1.58700 & -0.31187 & 3.18195 \\ \mathrm{H} & 1.23577 & -2.71407 & 2.44030 \\ \mathrm{H} & 0.19704 & -3.83496 & 1.56326 \\ \mathrm{C} & -4.28702 & 1.47574 & 0.22018 \\ \mathrm{C} & -5.51559 & 2.04402 & -0.44707 \\ \mathrm{O} & -3.81556 & 1.85548 & 1.27134 \\ \mathrm{H} & -6.26665 & 1.25872 & -0.58037 \\ \mathrm{H} & -5.26326 & 2.42838 & -1.44101 \\ \mathrm{H} & -5.92511 & 2.84779 & 0.16538 \\ \mathrm{H} & 3.90600 & -1.50489 & -2.46818 \\ \mathrm{H} & -2.63433 & 0.88503 & -2.17242\end{array}$

Conformer 2

Energy: -1384.14100 Hartree (Rel: $2.4 \mathrm{kcal} / \mathrm{mol}$ ) XYZ coordinates for conf 2 :

\begin{tabular}{|c|c|c|c|}
\hline C & 1.11770 & 3.78682 & 0.36637 \\
\hline C & 1.32550 & 2.27613 & 0.60869 \\
\hline C & 0.87446 & 1.40780 & -0.58903 \\
\hline C & 0.53112 & -0.03176 & -0.13950 \\
\hline $\mathrm{C}$ & -0.31997 & -0.88196 & -1.10952 \\
\hline C & -1.87370 & -0.60285 & -1.14910 \\
\hline O & -1.98896 & 0.65665 & -1.84459 \\
\hline C & 2.77049 & 2.03592 & 1.04191 \\
\hline C & 1.99700 & 1.42731 & -1.63242 \\
\hline C & -2.58783 & -0.29928 & 0.21391 \\
\hline C & 3.21040 & 0.73388 & -1.03455 \\
\hline $\mathrm{C}$ & 2.97888 & -0.77268 & -0.77453 \\
\hline C & 1.74127 & -0.95658 & 0.16545 \\
\hline C & 1.11265 & -2.36158 & 0.09942 \\
\hline O & 0.04136 & -2.24872 & -0.85917 \\
\hline C & -2.91440 & -1.47607 & 1.16390 \\
\hline C & -1.73827 & -2.40042 & 1.27176 \\
\hline C & -0.60662 & -2.23084 & 1.97452 \\
\hline C & -2.59837 & -1.68857 & -1.95129 \\
\hline O & 3.61737 & 1.34797 & 0.22863 \\
\hline O & 4.13489 & -1.31416 & -0.12628 \\
\hline C & -0.42414 & -1.27312 & 3.12556 \\
\hline C & 0.60706 & -2.96603 & 1.44966 \\
\hline O & -3.86520 & 0.31321 & -0.09656 \\
\hline O & 3.21380 & 2.45923 & 2.08838 \\
\hline $\mathrm{H}$ & 1.46013 & 4.35944 & 1.23249 \\
\hline $\mathrm{H}$ & 1.66503 & 4.14033 & -0.51426 \\
\hline $\mathrm{H}$ & 0.05297 & 3.98829 & 0.21202 \\
\hline $\mathrm{H}$ & 0.72780 & 2.01675 & 1.49109 \\
\hline $\mathrm{H}$ & -0.03601 & 1.84912 & -1.00415 \\
\hline $\mathrm{H}$ & -0.02784 & 0.05614 & 0.79400 \\
\hline $\mathrm{H}$ & -0.01857 & -0.66707 & -2.14750 \\
\hline $\mathrm{H}$ & 2.25251 & 2.45544 & -1.91241 \\
\hline $\mathrm{H}$ & 1.70747 & 0.91670 & -2.55576 \\
\hline $\mathrm{H}$ & -2.00090 & 0.44938 & 0.74525 \\
\hline $\mathrm{H}$ & 4.09715 & 0.84274 & -1.66464 \\
\hline C & 2.91256 & -1.51131 & -2.11920 \\
\hline $\mathrm{H}$ & 2.11150 & -0.75922 & 1.17706 \\
\hline $\mathrm{H}$ & 1.82670 & -3.08305 & -0.30793 \\
\hline $\mathrm{H}$ & -3.78747 & -2.00727 & 0.76881 \\
\hline $\mathrm{H}$ & -3.21426 & -1.04006 & 2.12376 \\
\hline $\mathrm{H}$ & -1.71770 & -3.18021 & 0.51646 \\
\hline
\end{tabular}




$\begin{array}{lrrr}\mathrm{H} & -3.65371 & -1.42275 & -2.06248 \\ \mathrm{H} & -2.15548 & -1.77639 & -2.95171 \\ \mathrm{H} & -2.52321 & -2.66957 & -1.47782 \\ \mathrm{H} & 4.37150 & -0.69332 & 0.58504 \\ \mathrm{H} & 3.00776 & -2.58771 & -1.95709 \\ \mathrm{H} & 1.98413 & -1.33237 & -2.66434 \\ \mathrm{H} & -0.12265 & -1.82560 & 4.02652 \\ \mathrm{H} & 0.37390 & -0.54205 & 2.93957 \\ \mathrm{H} & -1.33778 & -0.72095 & 3.36468 \\ \mathrm{H} & 1.44431 & -2.96896 & 2.15864 \\ \mathrm{H} & 0.35620 & -4.01434 & 1.24771 \\ \mathrm{C} & -4.01403 & 1.63796 & 0.13202 \\ \mathrm{C} & -5.35043 & 2.11476 & -0.38362 \\ \mathrm{O} & -3.18795 & 2.34031 & 0.67920 \\ \mathrm{H} & -6.15061 & 1.43823 & -0.06995 \\ \mathrm{H} & -5.33163 & 2.11881 & -1.47933 \\ \mathrm{H} & -5.54594 & 3.12513 & -0.02234 \\ \mathrm{H} & 3.75395 & -1.20059 & -2.74764 \\ \mathrm{H} & -1.79956 & 0.49392 & -2.78294\end{array}$

Tweaking the relative energies of the two conformers within $2.15 \mathrm{kcal} / \mathrm{mol}$ considerably improves rmsd(13C) and also rmsd(J):

\begin{tabular}{|c|c|c|c|c|c|c|c|c|c|}
\hline & & Rel e & ergy & $\mathrm{cal} / \mathrm{mol}$ & : & $\begin{array}{c}\text { Conf } 1 \\
0.0\end{array}$ & $\begin{array}{c}\text { Conf } 2 \\
2.4\end{array}$ & & \\
\hline iGau & jGau & Jexp & Jcalc & diff & & 1 & 2 & & \\
\hline 31 & 32 & 10.80 & 11.07 & 0.27 & {[} & 11.16 & $10.94]$ & $\mathrm{H} 1$ & $\mathrm{H} 2$ \\
\hline 35 & 40 & 9.90 & 10.12 & 0.22 & {[} & 9.92 & $10.43]$ & $\mathrm{H} 4$ & H5 \\
\hline 35 & 41 & 7.80 & 7.58 & -0.22 & {[} & 7.42 & $7.82]$ & H4 & H5 ' \\
\hline 39 & 53 & 6.60 & 6.74 & 0.14 & {[} & 6.84 & 6.58 & $\mathrm{H} 9$ & H8 \\
\hline 52 & 53 & 13.80 & 13.57 & -0.23 & {[} & -13.57 & $-13.58]$ & H8 ' & $\mathrm{H} 8$ \\
\hline 31 & 38 & 7.50 & 7.93 & 0.43 & {[} & 7.89 & $8.00]$ & $\mathrm{H1}$ & $\mathrm{H} 1 \mathrm{O}$ \\
\hline 33 & 36 & 4.20 & 4.80 & 0.60 & {[} & 4.80 & 4.80 & $\mathrm{H} 13$ & $\mathrm{H} 12$ \\
\hline 26 & 29 & 7.30 & 7.56 & 0.26 & {[} & 7.56 & $7.55]$ & $\mathrm{H} 17$ & $\mathrm{H} 15$ \\
\hline & & For $\mathrm{J}$ & rmsc & $=0.33 \mathrm{~Hz}$ & $\mathrm{~N}=8$ & $\{-0.2$ & $230.60\}$ & & \\
\hline
\end{tabular}

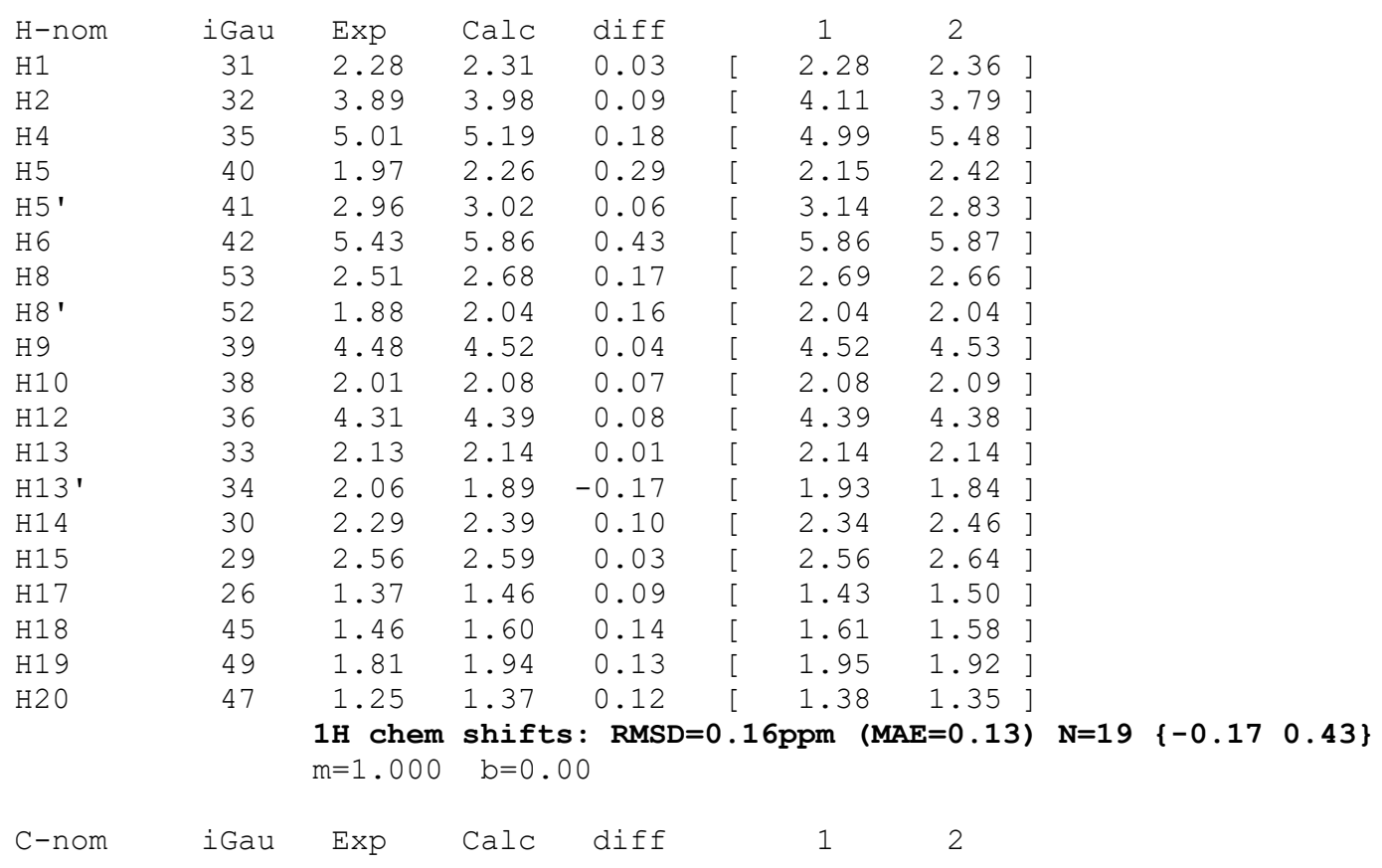




\begin{tabular}{|c|c|c|c|c|c|c|c|}
\hline $\mathrm{C} 1-\mathrm{CH}$ & 4 & 45.25 & 46.96 & 1.71 & 46.87 & $47.10]$ & \\
\hline $\mathrm{C} 2-\mathrm{CH}$ & 5 & 87.13 & 88.28 & 1.15 & 87.18 & $89.92]$ & \\
\hline C3-C & 6 & 76.88 & 78.45 & 1.57 & 78.07 & $79.02]$ & \\
\hline $\mathrm{C} 4-\mathrm{CH}$ & 10 & 73.79 & 75.78 & 1.99 & 78.34 & $71.94]$ & \\
\hline $\mathrm{C} 5-\mathrm{CH} 2$ & 16 & 28.59 & 29.23 & 0.64 & 28.86 & $29.78]$ & \\
\hline $\mathrm{C} 6-\mathrm{CH}$ & 17 & 123.53 & 125.09 & 1.56 & [ 125.07 & $125.12]$ & \\
\hline $\mathrm{C} 7-\mathrm{C}$ & 18 & 131.55 & 133.94 & 2.39 & [ 134.07 & $133.75]$ & \\
\hline $\mathrm{C} 8-\mathrm{CH} 2$ & 23 & 43.97 & 43.30 & -0.67 & [ 43.24 & $43.38]$ & \\
\hline $\mathrm{C} 9-\mathrm{CH}$ & 14 & 78.70 & 79.95 & 1.25 & 79.79 & 80.19 ] & \\
\hline $\mathrm{ClO}-\mathrm{CH}$ & 13 & 50.59 & 51.20 & 0.61 & 51.34 & $50.99]$ & \\
\hline C11-C & 12 & 72.17 & 73.38 & 1.21 & 73.42 & $73.33]$ & \\
\hline $\mathrm{C} 12-\mathrm{CH}$ & 11 & 83.55 & 84.83 & 1.28 & 84.87 & $84.78]$ & \\
\hline $\mathrm{C} 13-\mathrm{CH} 2$ & 9 & 19.68 & 20.18 & 0.50 & 20.21 & $20.14]$ & \\
\hline $\mathrm{Cl} 4-\mathrm{CH}$ & 3 & 31.15 & 31.43 & 0.28 & 31.54 & $31.27]$ & \\
\hline $\mathrm{C} 15-\mathrm{CH}$ & 2 & 43.94 & 44.95 & 1.01 & [ $\quad 44.94$ & $44.97]$ & \\
\hline $\mathrm{C} 16-\mathrm{C}$ & 8 & 174.25 & 173.38 & -0.87 & [ 173.39 & 173.37 ] & \\
\hline $\mathrm{C} 17-\mathrm{CH} 3$ & 1 & 19.43 & 18.91 & -0.52 & [ 18.92 & $18.89]$ & \\
\hline $\mathrm{C} 18-\mathrm{CH} 3$ & 19 & 22.41 & 22.84 & 0.43 & 22.84 & $22.83]$ & \\
\hline C19-CH3 & 22 & 19.15 & 18.09 & -1.06 & 18.15 & $18.00]$ & \\
\hline \multirow[t]{5}{*}{$\mathrm{C} 2 \mathrm{O}-\mathrm{CH} 2$} & 37 & 21.76 & 21.78 & 0.02 & 21.75 & $21.83]$ & \\
\hline & & $13 \mathrm{C} \mathrm{ch}$ & lem shi & Es: RMS & $1.19 \mathrm{ppm}$ & $(\mathrm{MAE}=1.04)$ & \multirow[t]{4}{*}{$\mathrm{N}=20 \quad \begin{cases}-1.06 & 2.39\end{cases}$} \\
\hline & & & & Fractio & $: 0.600$ & 0.400 & \\
\hline & Fracti & ns-der & ried re & energi & 0.00 & 0.24 & \\
\hline & & & hergy $\mathrm{d}$ & crepan & 0.00 & 2.15 & \\
\hline
\end{tabular}




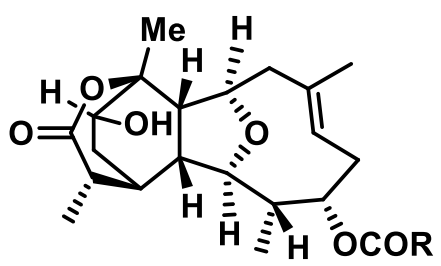

The best-fitting procedure, based on multiplicity of $13 \mathrm{C}$ peaks, was used and yet, the C11-C14 candidate structure gave an inferior rmsd.

$$
\text { Rel energy (kcal/mol): } \begin{array}{ccc}
\text { Conf } 1 & \text { Conf } 2 \\
0.0 & 1.7
\end{array}
$$

\begin{tabular}{|c|c|c|c|c|c|c|c|}
\hline C-nom & iGau & Exp & $\mathrm{Calc}$ & diff & 1 & 2 & \\
\hline$C-C$ & 8 & 174.25 & 174.16 & -0.09 & {$[174.17$} & 174.06 ] & \\
\hline$C-C$ & 54 & 173.15 & 173.64 & 0.49 & {$[173.64$} & $173.68]$ & \\
\hline $\mathrm{C}-\mathrm{C}$ & 18 & 131.55 & 134.15 & 2.60 & {$[133.68$} & $142.19]$ & \\
\hline$C-C$ & 12 & 76.88 & 84.47 & 7.59 & {$\left[\begin{array}{l}84.48 \\
0\end{array}\right.$} & $84.34]$ & \\
\hline$C-C$ & 6 & 72.17 & 77.57 & 5.40 & 77.51 & $78.66]$ & \\
\hline $\mathrm{C}-\mathrm{CH}$ & 17 & 123.53 & 124.76 & 1.23 & [ 125.29 & $115.73]$ & \\
\hline $\mathrm{C}-\mathrm{CH}$ & 5 & 87.13 & 96.38 & 9.25 & [ 96.37 & $96.55]$ & \\
\hline $\mathrm{C}-\mathrm{CH}$ & 14 & 83.55 & 83.48 & -0.07 & 83.50 & $83.07]$ & \\
\hline $\mathrm{C}-\mathrm{CH}$ & 10 & 78.70 & 79.55 & 0.85 & 79.47 & $80.99]$ & \\
\hline $\mathrm{C}-\mathrm{CH}$ & 11 & 73.79 & 72.24 & -1.55 & 72.24 & $72.18]$ & \\
\hline $\mathrm{C}-\mathrm{CH}$ & 13 & 50.59 & 49.77 & -0.82 & 49.39 & $56.23]$ & \\
\hline $\mathrm{C}-\mathrm{CH}$ & 4 & 43.94 & 46.35 & 2.41 & 46.40 & $45.45]$ & \\
\hline $\mathrm{C}-\mathrm{CH}$ & 2 & 39.43 & 46.11 & 6.68 & 46.11 & $46.20]$ & \\
\hline $\mathrm{C}-\mathrm{CH}$ & 3 & 31.15 & 38.21 & 7.06 & 38.20 & $38.34]$ & \\
\hline $\mathrm{C}-\mathrm{CH} 2$ & 23 & 43.97 & 40.85 & -3.12 & 40.79 & $41.85]$ & \\
\hline $\mathrm{C}-\mathrm{CH} 2$ & 16 & 28.59 & 30.07 & 1.48 & 29.99 & $31.48]$ & \\
\hline $\mathrm{C}-\mathrm{CH} 2$ & 55 & 27.30 & 27.30 & 0.00 & 27.29 & $27.50]$ & \\
\hline $\mathrm{C}-\mathrm{CH} 2$ & 9 & 19.68 & 26.53 & 6.85 & 26.53 & $26.49]$ & \\
\hline $\mathrm{C}-\mathrm{CH} 3$ & 19 & 22.41 & 23.32 & 0.91 & 23.38 & $22.38]$ & \\
\hline $\mathrm{C}-\mathrm{CH} 3$ & 37 & 21.76 & 22.84 & 1.08 & 22.84 & $22.79]$ & \\
\hline $\mathrm{C}-\mathrm{CH} 3$ & 22 & 19.43 & 19.73 & 0.30 & 19.59 & $22.16]$ & \\
\hline $\mathrm{C}-\mathrm{CH} 3$ & 1 & 19.15 & 17.96 & -1.19 & 17.95 & $18.07]$ & \\
\hline $\mathrm{C}-\mathrm{CH} 3$ & 57 & 8.30 & 8.32 & 0.02 & 8.32 & $8.29]$ & \\
\hline
\end{tabular}

\section{Conformer 1}

$\begin{array}{lrrr}\text { Energy: - } 1423.44841 & \text { Hartree (Rel: } & 0.0 \mathrm{kcal} / \mathrm{mol}) \\ \text { XYZ coordinates for } & \text { conf } 1: & \\ \text { C } & -3.02581 & 3.58426 & 0.78419 \\ \text { C } & -2.08286 & 2.53829 & 0.15432 \\ \text { C } & -1.75555 & 1.36395 & 1.09933 \\ \text { C } & -0.75658 & 0.37325 & 0.45448 \\ \text { C } & -0.02766 & -0.48465 & 1.51785 \\ \text { C } & 1.53044 & -0.28141 & 1.61318 \\ \text { O } & 1.57450 & 1.11645 & 1.94797 \\ \text { C } & -2.58762 & 2.20140 & -1.25172 \\ \text { C } & -2.99032 & 0.60621 & 1.60730 \\ \text { C } & 2.38878 & -0.46488 & 0.31109 \\ \text { C } & -3.80722 & 0.00508 & 0.45789 \\ \text { C } & -2.92269 & -0.27215 & -0.79759 \\ \text { C } & -1.46126 & -0.65353 & -0.47767 \\ \text { C } & -1.30570 & -2.03531 & 0.21956\end{array}$




\begin{tabular}{|c|c|c|}
\hline-0.46925 & -1.82997 & 1.36736 \\
\hline 2.84146 & -1.88777 & -0.09548 \\
\hline 1.62284 & -2.75700 & -0.15421 \\
\hline 0.69217 & -2.78460 & -1.12311 \\
\hline 2.10029 & -1.09492 & 2.78480 \\
\hline-4.42763 & -1.17147 & 0.97401 \\
\hline-2.90665 & 0.94104 & -1.62943 \\
\hline 0.91246 & -2.33539 & -2.54759 \\
\hline-0.70643 & -3.16142 & -0.68197 \\
\hline 3.59739 & 0.32755 & 0.55566 \\
\hline-2.70553 & 3.07456 & -2.08889 \\
\hline-3.11604 & 4.45104 & 0.12522 \\
\hline-4.03042 & 3.18267 & 0.95497 \\
\hline-2.62508 & 3.91664 & 1.74786 \\
\hline-1.13239 & 3.05292 & -0.04320 \\
\hline-1.25174 & 1.81652 & 1.96131 \\
\hline-0.02582 & 0.95719 & -0.11118 \\
\hline-0.35199 & -0.16120 & 2.51625 \\
\hline-3.62868 & 1.24212 & 2.22812 \\
\hline-2.66491 & -0.22186 & 2.24787 \\
\hline 1.87287 & -0.00475 & -0.53375 \\
\hline-4.58236 & 0.71066 & 0.12485 \\
\hline-3.59126 & -1.28002 & -1.73479 \\
\hline-0.94250 & -0.67185 & -1.43751 \\
\hline-2.27323 & -2.38408 & 0.59624 \\
\hline 3.56513 & -2.25803 & 0.63885 \\
\hline 3.36187 & -1.79797 & -1.05394 \\
\hline 1.34118 & -3.19507 & 0.79739 \\
\hline 3.17020 & -0.89161 & 2.90625 \\
\hline 1.59108 & -0.80245 & 3.70842 \\
\hline 1.96687 & -2.17105 & 2.64618 \\
\hline-5.14603 & -1.42892 & 0.37492 \\
\hline-3.01398 & -1.36941 & -2.65984 \\
\hline-3.66627 & -2.26722 & -1.27345 \\
\hline 0.75516 & -3.18277 & -3.23046 \\
\hline 0.20695 & -1.55774 & -2.86540 \\
\hline 1.92419 & -1.95623 & -2.71656 \\
\hline-1.38299 & -3.33313 & -1.52761 \\
\hline-0.69855 & -4.08168 & -0.08528 \\
\hline 4.19463 & 0.91609 & -0.51274 \\
\hline 5.41972 & 1.69677 & -0.08040 \\
\hline 3.78601 & 0.81450 & -1.65057 \\
\hline 6.16107 & 2.34191 & -1.25006 \\
\hline 6.07085 & 1.01157 & 0.47682 \\
\hline 5.09517 & 2.45144 & 0.64798 \\
\hline 7.03346 & 2.89324 & -0.88552 \\
\hline 5.51396 & 3.03945 & -1.79022 \\
\hline 6.50527 & 1.58636 & -1.96292 \\
\hline-4.59822 & -0.93949 & -2.00163 \\
\hline 2.50864 & 1.37834 & 1.88995 \\
\hline
\end{tabular}

Conformer 2

Energy: -1423.44572 Hartree (Rel: $1.7 \mathrm{kcal} / \mathrm{mol}$ ) XYZ coordinates for conf 2 :

$\begin{array}{lrrr}\text { C } & 2.94584 & -3.59930 & 1.07108 \\ \text { C } & 2.06849 & -2.59015 & 0.30191 \\ \text { C } & 1.70286 & -1.34212 & 1.13151 \\ \text { C } & 0.77122 & -0.38903 & 0.34363 \\ \text { C } & -0.01089 & 0.56183 & 1.28263 \\ \text { C } & -1.57861 & 0.41317 & 1.30924 \\ \text { O } & -1.69078 & -0.86652 & 1.96169 \\ \text { C } & 2.67413 & -2.37076 & -1.08812 \\ \text { C } & 2.91542 & -0.56583 & 1.66514\end{array}$




\begin{tabular}{|c|c|c|c|}
\hline $\mathrm{C}$ & -2.36993 & 0.27400 & -0.03779 \\
\hline C & 3.82202 & -0.07093 & 0.53262 \\
\hline $\mathrm{C}$ & 3.03133 & 0.12330 & -0.79958 \\
\hline C & 1.55980 & 0.55114 & -0.61216 \\
\hline $\mathrm{C}$ & 1.39369 & 1.98507 & -0.03071 \\
\hline O & 0.47103 & 1.88362 & 1.06636 \\
\hline C & -2.64384 & 1.52441 & -0.90400 \\
\hline C & -1.32618 & 2.02131 & -1.41461 \\
\hline C & -0.63733 & 3.08542 & -0.97702 \\
\hline C & -2.18184 & 1.50164 & 2.20569 \\
\hline O & 4.43415 & 1.13048 & 0.99946 \\
\hline O & 3.04734 & -1.14882 & -1.53677 \\
\hline $\mathrm{C}$ & -1.21452 & 4.23683 & -0.19621 \\
\hline C & 0.87521 & 3.04707 & -1.06256 \\
\hline O & -3.66896 & -0.27271 & 0.36945 \\
\hline 0 & 2.82824 & -3.30735 & -1.84700 \\
\hline $\mathrm{H}$ & 3.05927 & -4.51652 & 0.48844 \\
\hline $\mathrm{H}$ & 3.94576 & -3.20178 & 1.27561 \\
\hline $\mathrm{H}$ & 2.47734 & -3.84829 & 2.02929 \\
\hline $\mathrm{H}$ & 1.12321 & -3.10385 & 0.07877 \\
\hline $\mathrm{H}$ & 1.13172 & -1.71865 & 1.98808 \\
\hline $\mathrm{H}$ & 0.07419 & -1.00506 & -0.23095 \\
\hline $\mathrm{H}$ & 0.23550 & 0.31107 & 2.32291 \\
\hline $\mathrm{H}$ & 3.49587 & -1.16026 & 2.37730 \\
\hline $\mathrm{H}$ & 2.56247 & 0.31545 & 2.21361 \\
\hline $\mathrm{H}$ & -1.89226 & -0.48916 & -0.65542 \\
\hline $\mathrm{H}$ & 4.60129 & -0.81418 & 0.30963 \\
\hline C & 3.77959 & 1.04877 & -1.76111 \\
\hline $\mathrm{H}$ & 1.11051 & 0.51133 & -1.60777 \\
\hline $\mathrm{H}$ & 2.34299 & 2.33040 & 0.38988 \\
\hline $\mathrm{H}$ & -3.18096 & 2.27214 & -0.31455 \\
\hline $\mathrm{H}$ & -3.30670 & 1.20299 & -1.71684 \\
\hline $\mathrm{H}$ & -0.79170 & 1.29103 & -2.02289 \\
\hline $\mathrm{H}$ & -3.25983 & 1.34665 & 2.32175 \\
\hline $\mathrm{H}$ & -1.71936 & 1.45477 & 3.19669 \\
\hline $\mathrm{H}$ & -2.01384 & 2.49929 & 1.79571 \\
\hline $\mathrm{H}$ & 5.20012 & 1.32512 & 0.43664 \\
\hline $\mathrm{H}$ & 3.26519 & 1.07773 & -2.72619 \\
\hline $\mathrm{H}$ & 3.84318 & 2.06627 & -1.36908 \\
\hline $\mathrm{H}$ & -0.96232 & 5.18306 & -0.69528 \\
\hline $\mathrm{H}$ & -0.77818 & 4.28419 & 0.80904 \\
\hline $\mathrm{H}$ & -2.30302 & 4.19362 & -0.10388 \\
\hline $\mathrm{H}$ & 1.22881 & 2.78051 & -2.06493 \\
\hline $\mathrm{H}$ & 1.30244 & 4.02745 & -0.82400 \\
\hline C & -4.29598 & -1.12285 & -0.48449 \\
\hline $\mathrm{C}$ & -5.62977 & -1.56589 & 0.08343 \\
\hline O & -3.83565 & -1.46254 & -1.55428 \\
\hline C & -6.36612 & -2.55298 & -0.82039 \\
\hline $\mathrm{H}$ & -6.22876 & -0.66284 & 0.25819 \\
\hline $\mathrm{H}$ & -5.44854 & -1.99437 & 1.07740 \\
\hline $\mathrm{H}$ & -7.32449 & -2.83258 & -0.37171 \\
\hline $\mathrm{H}$ & -5.77864 & -3.46381 & -0.97123 \\
\hline $\mathrm{H}$ & -6.56105 & -2.11567 & -1.80423 \\
\hline $\mathrm{H}$ & 4.79456 & 0.67446 & -1.93628 \\
\hline $\mathrm{H}$ & -2.63521 & -1.09454 & 1.94627 \\
\hline
\end{tabular}


Briarellin J - original

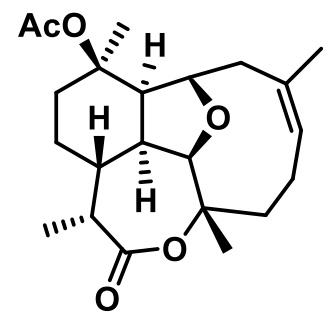

(the best fit to the experimental data still gives unacceptable rmsd $>2.6 \mathrm{ppm}$ )

$$
\text { Rel energy (kcal/mol): } \begin{array}{cc}
0.4 & 0.0
\end{array}
$$

\begin{tabular}{|c|c|c|c|c|c|c|c|}
\hline C-nom & iGau & $\operatorname{Exp}$ & Calc & diff & 1 & 2 & \\
\hline$C-C$ & 8 & 176.20 & 177.35 & 1.15 & [ 182.62 & $174.50 \quad]$ & \\
\hline$C-C$ & 19 & 131.50 & 131.45 & -0.05 & [ 129.93 & 132.27 ] & \\
\hline $\mathrm{C}-\mathrm{C}$ & 6 & 85.40 & 86.91 & 1.51 & 85.72 & $87.56]$ & \\
\hline$C-C$ & 13 & 81.40 & 84.10 & 2.70 & 84.13 & $84.09]$ & \\
\hline $\mathrm{C}-\mathrm{CH}$ & 18 & 129.70 & 129.59 & -0.11 & {$[130.65$} & $129.01]$ & \\
\hline $\mathrm{C}-\mathrm{CH}$ & 5 & 91.90 & 90.72 & -1.18 & [ 90.78 & $90.68]$ & \\
\hline $\mathrm{C}-\mathrm{CH}$ & 15 & 80.60 & 81.26 & 0.66 & 82.30 & $80.70]$ & \\
\hline $\mathrm{C}-\mathrm{CH}$ & 14 & 50.20 & 51.33 & 1.13 & 51.27 & $51.36]$ & \\
\hline $\mathrm{C}-\mathrm{CH}$ & 2 & 46.20 & 47.89 & 1.69 & 47.88 & $47.90]$ & \\
\hline $\mathrm{C}-\mathrm{CH}$ & 4 & 45.70 & 42.55 & -3.15 & 43.96 & $41.79]$ & \\
\hline $\mathrm{C}-\mathrm{CH}$ & 3 & 37.40 & 35.97 & -1.43 & 39.34 & $34.14]$ & \\
\hline $\mathrm{C}-\mathrm{CH} 2$ & 10 & 38.50 & 37.85 & -0.65 & 38.72 & $37.38]$ & \\
\hline $\mathrm{C}-\mathrm{CH} 2$ & 43 & 34.40 & 37.98 & 3.58 & 37.45 & $38.27]$ & \\
\hline $\mathrm{C}-\mathrm{CH} 2$ & 12 & 30.20 & 30.41 & 0.21 & 29.64 & $30.82]$ & \\
\hline $\mathrm{C}-\mathrm{CH} 2$ & 9 & 22.90 & 23.65 & 0.75 & 23.23 & $23.87]$ & \\
\hline $\mathrm{C}-\mathrm{CH} 2$ & 17 & 16.80 & 23.71 & 6.91 & 22.96 & $24.11]$ & \\
\hline $\mathrm{C}-\mathrm{CH} 3$ & 42 & 28.70 & 29.11 & 0.41 & 29.07 & $29.13]$ & \\
\hline $\mathrm{C}-\mathrm{CH} 3$ & 27 & 26.50 & 27.66 & 1.16 & 26.97 & $28.03]$ & \\
\hline $\mathrm{C}-\mathrm{CH} 3$ & 34 & 23.20 & 24.11 & 0.91 & 24.17 & 24.07 ] & \\
\hline \multirow[t]{2}{*}{$\mathrm{C}-\mathrm{CH} 3$} & 1 & 17.70 & 11.14 & -6.56 & {$[\quad 9.68$} & $11.93]$ & \\
\hline & & $13 \mathrm{C} \mathrm{cl}$ & lem shif & s: RMS & $2.61 \mathrm{ppm}$ & $(\mathrm{MAE}=1.80)$ & $\mathrm{N}=20 \quad\left\{\begin{array}{ll}-6.56 & 6.91\end{array}\right\}$ \\
\hline
\end{tabular}

Conformer 1

Energy: -1233.71399 Hartree (Rel: $0.4 \mathrm{kcal} / \mathrm{mol}$ ) XYZ coordinates for conf 1 :

$\begin{array}{lrrr}\text { C } & 1.10025 & -2.55622 & 2.66768 \\ \text { C } & 0.25010 & -2.67982 & 1.39290 \\ \text { C } & -0.63127 & -1.42902 & 1.06044 \\ \text { C } & 0.18499 & -0.22266 & 0.55133 \\ \text { C } & 0.77117 & -0.44766 & -0.85253 \\ \text { C } & 2.21383 & -1.01503 & -0.86606 \\ \text { O } & 2.19777 & -2.16445 & 0.06393 \\ \text { C } & 1.08999 & -2.96878 & 0.15239 \\ \text { C } & -1.52022 & -0.96517 & 2.22196 \\ \text { C } & 3.31192 & -0.11564 & -0.25055 \\ \text { O } & 0.81549 & -3.80177 & -0.68349 \\ \text { C } & -2.55042 & 0.09613 & 1.75742 \\ \text { C } & -2.16111 & 0.89724 & 0.48719 \\ \text { C } & -0.63158 & 1.09851 & 0.42270 \\ \text { C } & -0.11367 & 1.74921 & -0.89238 \\ \text { O } & 0.71642 & 0.78282 & -1.55143\end{array}$




$\begin{array}{rrrr}\mathrm{C} & 3.41327 & 1.36594 & -0.72671 \\ \mathrm{C} & 2.89670 & 2.33223 & 0.31371 \\ \mathrm{C} & 1.76687 & 3.05918 & 0.34174 \\ \mathrm{H} & 0.45546 & -2.44790 & 3.54535 \\ \mathrm{H} & 1.70824 & -3.45588 & 2.81304 \\ \mathrm{H} & 1.77882 & -1.70016 & 2.62841 \\ \mathrm{H} & -0.43374 & -3.52861 & 1.50521 \\ \mathrm{H} & -1.29278 & -1.74828 & 0.24682 \\ \mathrm{H} & 1.00018 & -0.03198 & 1.25590 \\ \mathrm{H} & 0.13889 & -1.17264 & -1.38981 \\ \mathrm{C} & 2.59266 & -1.49051 & -2.26870 \\ \mathrm{H} & -0.88722 & -0.54550 & 3.01369 \\ \mathrm{H} & -2.04853 & -1.81695 & 2.66662 \\ \mathrm{H} & 4.26026 & -0.63147 & -0.44132 \\ \mathrm{H} & 3.18386 & -0.13023 & 0.83655 \\ \mathrm{H} & -3.52282 & -0.36423 & 1.57053 \\ \mathrm{H} & -2.71016 & 0.81970 & 2.56497 \\ \mathrm{C} & -2.90295 & 2.23956 & 0.44382 \\ \mathrm{O} & -2.45955 & 0.11969 & -0.73428 \\ \mathrm{H} & -0.39000 & 1.74294 & 1.27323 \\ \mathrm{H} & -0.95327 & 1.95785 & -1.56574 \\ \mathrm{H} & -2.73834 & 2.75384 & -0.50867 \\ \mathrm{H} & 2.90988 & 1.50230 & -1.68415 \\ \mathrm{H} & 4.47621 & 1.59127 & -0.88616 \\ \mathrm{H} & 3.55837 & 2.43198 & 1.17740 \\ \mathrm{C} & 1.49436 & 3.98917 & 1.50511 \\ \mathrm{C} & 0.69528 & 3.05336 & -0.73856 \\ \mathrm{H} & 2.27727 & 3.93218 & 2.26825 \\ \mathrm{H} & 0.53183 & 3.76644 & 1.98734 \\ \mathrm{H} & 1.42928 & 5.03224 & 1.16365 \\ \mathrm{H} & -1.13331 & 3.26381 & -1.72273 \\ \mathrm{H} & -0.01557 & 3.86611 & -0.54211 \\ \mathrm{H} & -2.53570 & 2.88531 & 1.24864 \\ \mathrm{H} & -3.97633 & 2.09528 & 0.57477 \\ \mathrm{C} & -3.71307 & -0.22636 & -1.09621 \\ \mathrm{C} & -3.68665 & -1.00343 & -2.39359 \\ \mathrm{O} & -4.72152 & 0.03672 & -0.47025 \\ \mathrm{H} & -3.17576 & -0.42778 & -3.17174 \\ \mathrm{H} & -3.12973 & -1.93704 & -2.25902 \\ \mathrm{H} & -4.70751 & -1.22785 & -2.70510 \\ & -1.86044 & -1.97107 & -2.25599 \\ \mathrm{H} & -2.21032 & -2.64567 \\ \mathrm{H} & -0.63875 & -2.95670\end{array}$

Conformer 2

Energy: -1233.71457 Hartree (Rel: $0.0 \mathrm{kcal} / \mathrm{mol}$ ) XYZ coordinates for conf 2 :

$\begin{array}{lrrr}\text { C } & 1.80371 & -1.76719 & 2.62398 \\ \text { C } & 0.87667 & -2.29431 & 1.50457 \\ \text { C } & -0.30034 & -1.36731 & 1.12638 \\ C & 0.17225 & -0.07325 & 0.46650 \\ C & 0.55719 & -0.28407 & -0.99259 \\ C & 1.99947 & -0.76274 & -1.22718 \\ \text { O } & 2.13534 & -2.15463 & -0.75917 \\ C & 1.74261 & -2.84025 & 0.34722 \\ C & -1.21828 & -1.00565 & 2.30351 \\ C & 3.12351 & 0.06702 & -0.56502 \\ \text { O } & 2.14055 & -3.98809 & 0.41935 \\ C & -2.48980 & -0.26911 & 1.81481 \\ C & -2.31517 & 0.60934 & 0.53997 \\ C & -0.85325 & 1.08722 & 0.43060 \\ C & -0.45944 & 1.85058 & -0.86418 \\ \text { O } & 0.34343 & 0.95712 & -1.65530\end{array}$




\begin{tabular}{|c|c|c|}
\hline 3.11890 & 1.60224 & -0.85087 \\
\hline 2.59270 & 2.43668 & 0.29268 \\
\hline 1.43826 & 3.11697 & 0.40429 \\
\hline 1.23188 & -1.60666 & 3.54274 \\
\hline 2.58118 & -2.50675 & 2.83831 \\
\hline 2.29618 & -0.82463 & 2.36748 \\
\hline 0.43332 & -3.21690 & 1.89437 \\
\hline-0.91244 & -1.92149 & 0.40240 \\
\hline 1.03814 & 0.30814 & 1.01199 \\
\hline-0.10199 & -1.04149 & -1.44213 \\
\hline 2.25226 & -0.90236 & -2.73283 \\
\hline-0.67080 & -0.36805 & 3.00905 \\
\hline-1.51218 & -1.90371 & 2.86061 \\
\hline 4.06472 & -0.36803 & -0.91912 \\
\hline 3.11353 & -0.09763 & 0.51701 \\
\hline-3.29278 & -0.98210 & 1.61652 \\
\hline-2.85668 & 0.37886 & 2.61889 \\
\hline-3.29753 & 1.78682 & 0.54417 \\
\hline-2.50657 & -0.19994 & -0.68085 \\
\hline-0.69689 & 1.73114 & 1.30160 \\
\hline-1.34505 & 2.09273 & -1.46280 \\
\hline-3.27034 & 2.32851 & -0.40691 \\
\hline 2.57221 & 1.81432 & -1.77073 \\
\hline 4.16074 & 1.90435 & -1.02188 \\
\hline 3.27371 & 2.48994 & 1.14521 \\
\hline 1.16456 & 3.94022 & 1.64562 \\
\hline 0.33622 & 3.15867 & -0.64734 \\
\hline 1.96727 & 3.84904 & 2.38444 \\
\hline 0.22179 & 3.64863 & 2.12933 \\
\hline 1.05897 & 5.00427 & 1.39013 \\
\hline 0.74248 & 3.43891 & -1.62742 \\
\hline-0.38057 & 3.94458 & -0.37743 \\
\hline-3.02487 & 2.48502 & 1.34290 \\
\hline-4.31827 & 1.44185 & 0.71502 \\
\hline-3.68506 & -0.77732 & -0.99682 \\
\hline-3.56937 & -1.51957 & -2.30965 \\
\hline-4.69532 & -0.72067 & -0.32309 \\
\hline-3.21431 & -0.84697 & -3.09667 \\
\hline-2.83861 & -2.33040 & -2.21760 \\
\hline-4.54141 & -1.93257 & -2.58186 \\
\hline 3.22800 & -1.36515 & -2.90809 \\
\hline 1.48272 & -1.53084 & -3.19248 \\
\hline 2.23042 & 0.07684 & -3.21733 \\
\hline
\end{tabular}




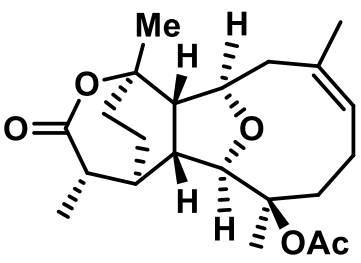

Conf 1 Conf 2 Conf 3

Rel energy ( $\mathrm{kcal} / \mathrm{mol}): \begin{array}{ccc}0.4 & 0.0 & 0.1\end{array}$

\begin{tabular}{|c|c|c|c|c|c|c|c|c|c|c|c|}
\hline iGau & jGau & Jexp & Jcalc & $\operatorname{diff}$ & & 1 & 2 & 3 & & & \\
\hline 47 & 48 & 14.10 & 15.12 & 1.02 & {[} & -14.43 & $-15 \cdot 30$ & -15.35 & ] & $\mathrm{H} 8 \mathrm{~b}$ & $\mathrm{H} 8 \mathrm{a}$ \\
\hline 23 & 24 & 4.70 & 3.83 & -0.87 & {[} & 1.25 & 4.50 & 4.69 & ] & H15 & H1 4 \\
\hline 20 & 23 & $\begin{array}{c}7.60 \\
\text { For Js }\end{array}$ & $\begin{array}{r}7.48 \\
\text { rmsc }\end{array}$ & $\begin{array}{l}-0.12 \\
=0.78 \mathrm{~Hz}\end{array}$ & $\mathrm{~N}=3$ & $\begin{array}{l}7.16 \\
\{-0 . \varepsilon\end{array}$ & $\begin{array}{l}7.58 \\
37 \\
1.02\end{array}$ & 7.58 & ] & $\mathrm{H} 17$ & $\mathrm{H} 15$ \\
\hline
\end{tabular}

\begin{tabular}{|c|c|c|c|c|c|c|c|c|}
\hline H-nom & iGau & Exp & Calc & $\operatorname{diff}$ & & 1 & 2 & 3 \\
\hline $\mathrm{H} 1$ & 25 & 2.66 & 2.73 & 0.07 & {[} & 2.82 & 2.75 & 2.66 \\
\hline $\mathrm{H} 2$ & 26 & 3.98 & 4.38 & 0.40 & {[} & 4.12 & 3.93 & 5.09 \\
\hline $\mathrm{H} 4 \mathrm{a}$ & 30 & 2.06 & 2.66 & 0.60 & {[} & 3.11 & 3.05 & 1.91 \\
\hline $\mathrm{H} 4 \mathrm{~b}$ & 31 & 1.82 & 1.60 & -0.22 & {[} & 1.80 & 1.44 & 1.67 \\
\hline $\mathrm{H} 5 \mathrm{a}$ & 39 & 1.86 & 2.04 & 0.18 & {[} & 2.03 & 2.05 & 2.03 \\
\hline $\mathrm{H} 5 \mathrm{~b}$ & 40 & 2.32 & 2.62 & 0.30 & {[} & 2.59 & 2.58 & 2.69 \\
\hline H6 & 41 & 5.64 & 6.03 & 0.39 & {[} & 5.90 & 6.06 & 6.09 \\
\hline $\mathrm{H} 8 \mathrm{a}$ & 48 & 2.16 & 1.95 & -0.21 & {[} & 1.93 & 1.96 & 1.94 \\
\hline $\mathrm{H} 8 \mathrm{~b}$ & 47 & 2.48 & 2.94 & 0.46 & {[} & 2.84 & 2.95 & 2.98 \\
\hline $\mathrm{H} 9$ & 37 & 4.02 & 4.30 & 0.28 & {[} & 4.33 & 4.30 & 4.28 \\
\hline H1O & 36 & 2.67 & 2.83 & 0.16 & {[} & 2.69 & 2.86 & 2.88 \\
\hline $\mathrm{H} 12 \mathrm{a}$ & 32 & 2.03 & 2.12 & 0.09 & {[} & 1.89 & 2.20 & 2.17 \\
\hline $\mathrm{H} 12 \mathrm{~b}$ & 33 & 2.03 & 2.22 & 0.19 & {[} & 2.17 & 2.25 & 2.23 \\
\hline $\mathrm{H} 13 \mathrm{a}$ & 28 & 1.87 & 2.04 & 0.17 & {[} & 1.91 & 2.09 & 2.06 \\
\hline $\mathrm{H} 13 \mathrm{~b}$ & 29 & 1.87 & 1.98 & 0.11 & {[} & 2.04 & 1.97 & 1.95 \\
\hline $\mathrm{H} 14$ & 24 & 1.67 & 1.74 & 0.07 & {[} & 2.04 & 1.68 & 1.63 \\
\hline H15 & 23 & 2.94 & 2.85 & -0.09 & {[} & 2.81 & 2.91 & 2.81 \\
\hline $\mathrm{H} 17$ & 20 & 1.36 & 1.49 & 0.13 & {[} & 1.40 & 1.52 & 1.50 \\
\hline $\mathrm{H} 18$ & 49 & 1.58 & 1.43 & -0.15 & {[} & 1.51 & 1.41 & 1.41 \\
\hline $\mathrm{H} 19$ & 45 & 1.76 & 1.90 & 0.14 & {[} & 1.92 & 1.89 & 1.90 \\
\hline H2O & 38 & 1.33 & 1.43 & 0.10 & {[} & 1.51 & 1.41 & 1.41 \\
\hline $\mathrm{H} 22$ & 55 & 1.99 & 1.98 & -0.01 & {[} & 1.98 & 1.97 & 2.00 \\
\hline
\end{tabular}

1H chem shifts: $\mathrm{RMSD}=0.25 \mathrm{ppm}(\mathrm{MAE}=0.21) \mathrm{N}=22\left\{\begin{array}{lll}-0.22 & 0.60\end{array}\right.$

\begin{tabular}{|c|c|c|c|c|c|c|c|}
\hline C-nom & iGau & Exp & Calc & $\operatorname{diff}$ & 1 & 2 & 3 \\
\hline $\mathrm{C} 1$ & 4 & 45.70 & 47.41 & 1.71 & 49.30 & 46.97 & 46.71 \\
\hline $\mathrm{C} 2$ & 5 & 91.90 & 89.80 & -2.10 & 86.71 & 92.79 & 88.20 \\
\hline C3 & 6 & 85.40 & 86.47 & 1.07 & 87.31 & 85.99 & 86.51 \\
\hline C4 & 10 & 34.40 & 33.51 & -0.89 & 31.86 & 31.31 & 37.21 \\
\hline C5 & 17 & 22.90 & 23.23 & 0.33 & [ 22.94 & 23.10 & 23.57 \\
\hline $\mathrm{C} 6$ & 18 & 129.70 & 130.67 & 0.97 & [ 129.76 & 131.03 & 130.83 \\
\hline C7 & 19 & 131.50 & 131.09 & -0.41 & [ 130.76 & 130.97 & 131.44 \\
\hline C8 & 43 & 38.50 & 37.78 & -0.72 & 38.36 & 37.60 & 37.62 \\
\hline C9 & 15 & 80.60 & 82.76 & 2.16 & 81.64 & 83.25 & 82.89 \\
\hline $\mathrm{C} 10$ & 14 & 50.20 & 49.64 & -0.56 & 51.82 & 48.73 & 49.34 \\
\hline C11 & 13 & 81.40 & 82.24 & 0.84 & 84.28 & 81.63 & 81.65 \\
\hline C12 & 12 & 30.20 & 29.95 & -0.25 & 29.41 & 30.02 & 30.22 \\
\hline C13 & 9 & 16.80 & 17.25 & 0.45 & 18.15 & 16.94 & 17.03 \\
\hline C14 & 3 & 37.40 & 36.50 & -0.90 & 32.84 & 38.40 & 36.57 \\
\hline C15 & 2 & 46.20 & 47.03 & 0.83 & 48.08 & 46.75 & 46.68 \\
\hline C1 6 & 8 & 176.20 & 174.35 & -1.85 & [ 173.82 & 174.45 & 174.56 \\
\hline
\end{tabular}




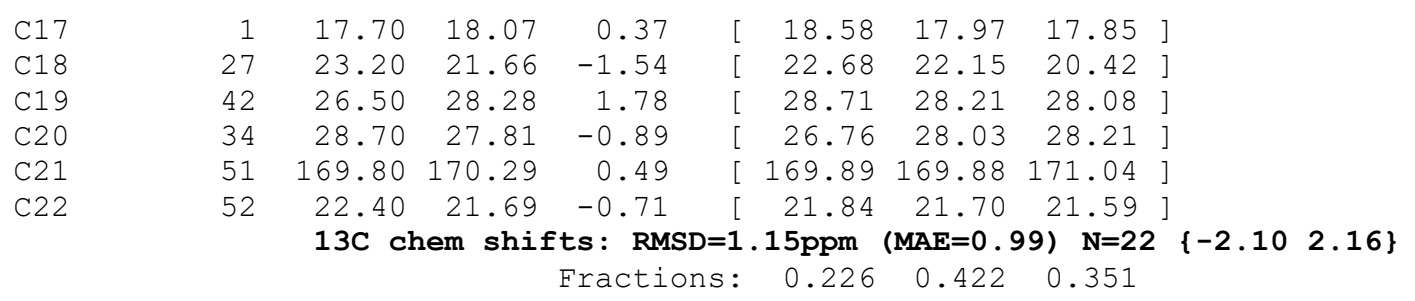

Conformer 1

Energy: -1233.72204 Hartree (Rel: $0.4 \mathrm{kcal} / \mathrm{mol}$ ) XYZ coordinates for conf 1 :

\begin{tabular}{|c|c|c|c|}
\hline C & 1.67291 & -3.33345 & -1.70123 \\
\hline C & 1.69391 & -1.88078 & -1.19421 \\
\hline $\mathrm{C}$ & 0.66250 & -1.64363 & -0.06399 \\
\hline C & 0.21830 & -0.16913 & -0.05080 \\
\hline C & -0.89133 & 0.17326 & 0.96884 \\
\hline C & -2.36845 & 0.14773 & 0.47908 \\
\hline O & 3.43478 & -0.57465 & 0.08723 \\
\hline C & 3.13865 & -1.47269 & -0.87949 \\
\hline C & 1.20157 & -2.10812 & 1.31017 \\
\hline C & -2.68007 & 0.95802 & -0.79860 \\
\hline O & 4.07255 & -1.90905 & -1.52467 \\
\hline $\mathrm{C}$ & 2.00165 & -1.00060 & 2.03302 \\
\hline C & 2.53396 & 0.06309 & 1.06823 \\
\hline C & 1.38898 & 0.79027 & 0.31344 \\
\hline C & 0.69395 & 1.94482 & 1.08355 \\
\hline O & -0.60630 & 1.47775 & 1.46358 \\
\hline C & -2.30184 & 2.47108 & -0.78225 \\
\hline C & -1.07041 & 2.79434 & -1.59091 \\
\hline $\mathrm{C}$ & 0.15328 & 3.15157 & -1.16780 \\
\hline $\mathrm{H}$ & 1.90192 & -4.04117 & -0.89627 \\
\hline $\mathrm{H}$ & 0.67963 & -3.57585 & -2.09340 \\
\hline $\mathrm{H}$ & 2.41017 & -3.47643 & -2.49330 \\
\hline $\mathrm{H}$ & 1.41415 & -1.24154 & -2.04644 \\
\hline $\mathrm{H}$ & -0.21413 & -2.24863 & -0.31391 \\
\hline $\mathrm{H}$ & -0.13476 & 0.09306 & -1.05211 \\
\hline $\mathrm{H}$ & -0.85583 & -0.53379 & 1.81058 \\
\hline $\mathrm{C}$ & -3.28033 & 0.53612 & 1.64961 \\
\hline $\mathrm{H}$ & 1.83306 & -2.99245 & 1.16634 \\
\hline $\mathrm{H}$ & 0.37568 & -2.43784 & 1.94874 \\
\hline $\mathrm{H}$ & -3.75378 & 0.85404 & -0.96811 \\
\hline $\mathrm{H}$ & -2.19096 & 0.46975 & -1.64977 \\
\hline $\mathrm{H}$ & 1.36610 & -0.48392 & 2.76283 \\
\hline $\mathrm{H}$ & 2.83670 & -1.42949 & 2.59723 \\
\hline C & 3.49779 & 1.02176 & 1.77354 \\
\hline O & -2.53150 & -1.28890 & 0.17501 \\
\hline $\mathrm{H}$ & 1.84360 & 1.19210 & -0.59582 \\
\hline $\mathrm{H}$ & 1.21837 & 2.18560 & 2.01403 \\
\hline $\mathrm{H}$ & 3.80157 & 1.83665 & 1.10781 \\
\hline $\mathrm{H}$ & -3.14085 & 3.02267 & -1.22692 \\
\hline $\mathrm{H}$ & -2.19911 & 2.82899 & 0.24365 \\
\hline $\mathrm{H}$ & -1.22014 & 2.73167 & -2.67101 \\
\hline $\mathrm{C}$ & 1.23411 & 3.49911 & -2.16925 \\
\hline C & 0.55899 & 3.26889 & 0.29379 \\
\hline $\mathrm{H}$ & 2.13945 & 2.89328 & -2.02542 \\
\hline $\mathrm{H}$ & 1.54751 & 4.54654 & -2.05310 \\
\hline $\mathrm{H}$ & 0.89475 & 3.36144 & -3.20093 \\
\hline $\mathrm{H}$ & -0.15412 & 3.88937 & 0.85098 \\
\hline $\mathrm{H}$ & 1.52550 & 3.78527 & 0.35423 \\
\hline $\mathrm{H}$ & 3.04983 & 1.45127 & 2.67318 \\
\hline $\mathrm{H}$ & 4.39471 & 0.47174 & 2.07245 \\
\hline C & -3.71336 & -1.83846 & -0.17620 \\
\hline
\end{tabular}




$\begin{array}{lrrr}\mathrm{C} & -3.55131 & -3.32710 & -0.39581 \\ \mathrm{O} & -4.76086 & -1.23612 & -0.30345 \\ \mathrm{H} & -2.83965 & -3.51216 & -1.20740 \\ \mathrm{H} & -4.51748 & -3.76534 & -0.64865 \\ \mathrm{H} & -3.15192 & -3.80197 & 0.50603 \\ \mathrm{H} & -4.33055 & 0.48832 & 1.36000 \\ \mathrm{H} & -3.11610 & -0.14011 & 2.49622 \\ \mathrm{H} & -3.04326 & 1.55010 & 1.98029\end{array}$

Conformer 2

Energy: -1233.72263 Hartree (Rel: $0.0 \mathrm{kcal} / \mathrm{mol}$ ) XYZ coordinates for conf 2 :

\begin{tabular}{|c|c|c|c|}
\hline C & 2.44343 & -3.73331 & 0.04452 \\
\hline C & 1.72719 & -2.44896 & -0.42096 \\
\hline $\mathrm{C}$ & 0.94665 & -1.73963 & 0.70596 \\
\hline C & 0.20547 & -0.48882 & 0.17692 \\
\hline $\mathrm{C}$ & -0.96092 & -0.06966 & 1.10465 \\
\hline $\mathrm{C}$ & -2.38308 & -0.07819 & 0.47061 \\
\hline O & 3.02778 & -0.33894 & -0.94521 \\
\hline $\mathrm{C}$ & 2.70633 & -1.61339 & -1.25528 \\
\hline C & 1.80817 & -1.36876 & 1.92132 \\
\hline C & -2.55072 & 0.86445 & -0.73877 \\
\hline 0 & 3.21465 & -2.08713 & -2.25362 \\
\hline C & 2.93490 & -0.39457 & 1.52557 \\
\hline C & 2.62093 & 0.41746 & 0.25363 \\
\hline $\mathrm{C}$ & 1.12509 & 0.76667 & 0.09028 \\
\hline $\mathrm{C}$ & 0.57427 & 1.75519 & 1.15160 \\
\hline 0 & -0.67944 & 1.23158 & 1.60415 \\
\hline C & -2.46567 & 2.39551 & -0.42606 \\
\hline $\mathrm{C}$ & -1.35959 & 3.08916 & -1.17892 \\
\hline C & -0.14572 & 3.44854 & -0.72937 \\
\hline $\mathrm{H}$ & 1.73562 & -4.39276 & 0.55861 \\
\hline $\mathrm{H}$ & 2.85380 & -4.26510 & -0.81720 \\
\hline $\mathrm{H}$ & 3.26782 & -3.51820 & 0.73255 \\
\hline $\mathrm{H}$ & 0.98103 & -2.75997 & -1.16553 \\
\hline $\mathrm{H}$ & 0.18220 & -2.45833 & 1.02771 \\
\hline $\mathrm{H}$ & -0.18727 & -0.72567 & -0.81563 \\
\hline $\mathrm{H}$ & -1.01307 & -0.76016 & 1.95936 \\
\hline $\mathrm{C}$ & -3.42702 & 0.15913 & 1.56706 \\
\hline $\mathrm{H}$ & 2.22612 & -2.26389 & 2.39251 \\
\hline $\mathrm{H}$ & 1.16324 & -0.90551 & 2.67782 \\
\hline $\mathrm{H}$ & -3.52150 & 0.63576 & -1.18319 \\
\hline $\mathrm{H}$ & -1.79707 & 0.60061 & -1.48923 \\
\hline $\mathrm{H}$ & 3.13709 & 0.29959 & 2.34841 \\
\hline $\mathrm{H}$ & 3.87640 & -0.92573 & 1.34344 \\
\hline $\mathrm{C}$ & 3.52731 & 1.64694 & 0.16853 \\
\hline O & -2.45704 & -1.47497 & -0.00128 \\
\hline $\mathrm{H}$ & 1.01284 & 1.21839 & -0.89914 \\
\hline $\mathrm{H}$ & 1.23953 & 1.79025 & 2.02679 \\
\hline $\mathrm{H}$ & 3.38728 & 2.16697 & -0.78385 \\
\hline $\mathrm{H}$ & -3.41878 & 2.85838 & -0.71078 \\
\hline $\mathrm{H}$ & -2.34271 & 2.55567 & 0.64520 \\
\hline $\mathrm{H}$ & -1.58241 & 3.30344 & -2.22579 \\
\hline C & 0.82530 & 4.15462 & -1.65044 \\
\hline C & 0.36448 & 3.21906 & 0.68841 \\
\hline $\mathrm{H}$ & 1.09783 & 5.14178 & -1.25075 \\
\hline $\mathrm{H}$ & 0.40716 & 4.29839 & -2.65183 \\
\hline $\mathrm{H}$ & 1.76517 & 3.59478 & -1.75315 \\
\hline $\mathrm{H}$ & -0.31242 & 3.67353 & 1.42331 \\
\hline $\mathrm{H}$ & 1.32139 & 3.74195 & 0.80132 \\
\hline $\mathrm{H}$ & 3.31982 & 2.34455 & 0.98505 \\
\hline $\mathrm{H}$ & 4.57597 & 1.34056 & 0.23996 \\
\hline $\mathrm{C}$ & -3.57679 & -2.01504 & -0.52640 \\
\hline
\end{tabular}




$\begin{array}{lrrr}\mathrm{C} & -3.33052 & -3.45965 & -0.90411 \\ \mathrm{O} & -4.63309 & -1.43447 & -0.68006 \\ \mathrm{H} & -3.02143 & -4.03297 & -0.02404 \\ \mathrm{H} & -2.52107 & -3.52389 & -1.63877 \\ \mathrm{H} & -4.24227 & -3.88759 & -1.32231 \\ \mathrm{H} & -4.43525 & 0.20469 & 1.15324 \\ \mathrm{H} & -3.38533 & -0.65269 & 2.30188 \\ \mathrm{H} & -3.20959 & 1.09425 & 2.08964\end{array}$

Conformer 3

Energy: -1233.72245 Hartree (Rel: $0.1 \mathrm{kcal} / \mathrm{mol}$ )

XYZ coordinates for conf 3 :

\begin{tabular}{|c|c|c|c|}
\hline C & 2.36317 & -3.66802 & -0.06716 \\
\hline $\mathrm{C}$ & 1.66319 & -2.37395 & -0.53154 \\
\hline C & 0.77464 & -1.73322 & 0.55602 \\
\hline C & 0.07909 & -0.45189 & 0.03216 \\
\hline $\mathrm{C}$ & -1.18140 & -0.09149 & 0.85713 \\
\hline C & -2.51982 & -0.00860 & 0.06472 \\
\hline O & 2.98175 & -0.23146 & -0.81976 \\
\hline C & 2.69218 & -1.48278 & -1.23746 \\
\hline $\mathrm{C}$ & 1.51675 & -1.43129 & 1.86553 \\
\hline C & -2.53309 & 1.06336 & -1.04729 \\
\hline $\mathrm{O}$ & 3.27572 & -1.88824 & -2.22474 \\
\hline $\mathrm{C}$ & 2.67897 & -0.44978 & 1.62751 \\
\hline C & 2.47285 & 0.44438 & 0.38814 \\
\hline $\mathrm{C}$ & 0.99542 & 0.80791 & 0.12280 \\
\hline C & 0.34917 & 1.70694 & 1.20964 \\
\hline O & -0.95574 & 1.17878 & 1.46773 \\
\hline C & -2.49288 & 2.55582 & -0.58915 \\
\hline $\mathrm{C}$ & -1.31640 & 3.31108 & -1.15275 \\
\hline $\mathrm{C}$ & -0.15122 & 3.60149 & -0.55082 \\
\hline $\mathrm{H}$ & 1.62776 & -4.36164 & 0.35487 \\
\hline $\mathrm{H}$ & 2.85410 & -4.15207 & -0.91490 \\
\hline $\mathrm{H}$ & 3.12271 & -3.47281 & 0.69714 \\
\hline $\mathrm{H}$ & 0.99177 & -2.66142 & -1.35273 \\
\hline $\mathrm{H}$ & -0.00751 & -2.47134 & 0.77023 \\
\hline $\mathrm{H}$ & -0.20505 & -0.62264 & -1.01094 \\
\hline $\mathrm{H}$ & -1.32808 & -0.82925 & 1.65225 \\
\hline $\mathrm{C}$ & -3.71089 & 0.15221 & 1.01395 \\
\hline $\mathrm{H}$ & 1.88329 & -2.35274 & 2.32873 \\
\hline $\mathrm{H}$ & 0.80292 & -1.00144 & 2.57851 \\
\hline $\mathrm{H}$ & -3.44211 & 0.87842 & -1.62998 \\
\hline $\mathrm{H}$ & -1.69844 & 0.86315 & -1.72832 \\
\hline $\mathrm{H}$ & 2.82035 & 0.18755 & 2.50700 \\
\hline $\mathrm{H}$ & 3.62783 & -0.98053 & 1.48650 \\
\hline C & 3.38175 & 1.67229 & 0.46160 \\
\hline O & -2.69241 & -1.24507 & -0.73037 \\
\hline $\mathrm{H}$ & 0.96451 & 1.33816 & -0.83336 \\
\hline $\mathrm{H}$ & 0.91767 & 1.63836 & 2.14937 \\
\hline $\mathrm{H}$ & 3.32135 & 2.25529 & -0.46211 \\
\hline $\mathrm{H}$ & -3.41270 & 3.04779 & -0.92923 \\
\hline $\mathrm{H}$ & -2.48394 & 2.61618 & 0.49911 \\
\hline $\mathrm{H}$ & -1.43515 & 3.64137 & -2.18637 \\
\hline C & 0.90822 & 4.39028 & -1.28894 \\
\hline C & 0.21439 & 3.21440 & 0.87808 \\
\hline $\mathrm{H}$ & 1.14405 & 5.32279 & -0.75671 \\
\hline $\mathrm{H}$ & 0.59090 & 4.65068 & -2.30366 \\
\hline $\mathrm{H}$ & 1.85143 & 3.83169 & -1.36185 \\
\hline $\mathrm{H}$ & -0.52366 & 3.61211 & 1.58681 \\
\hline $\mathrm{H}$ & 1.16372 & 3.69788 & 1.13557 \\
\hline $\mathrm{H}$ & 3.10645 & 2.31366 & 1.30362 \\
\hline $\mathrm{H}$ & 4.42099 & 1.35642 & 0.59917 \\
\hline C & -2.80724 & -2.47006 & -0.18163 \\
\hline
\end{tabular}




$\begin{array}{rrr}-3.12618 & -3.49318 & -1.24962 \\ -2.67485 & -2.72923 & 1.00006 \\ -2.40819 & -3.42476 & -2.07256 \\ -4.11997 & -3.29484 & -1.66562 \\ -3.10556 & -4.49363 & -0.81586 \\ -4.64686 & 0.17338 & 0.44517 \\ -3.75153 & -0.66875 & 1.73208 \\ -3.62292 & 1.08944 & 1.56924\end{array}$


Briarellins K - original

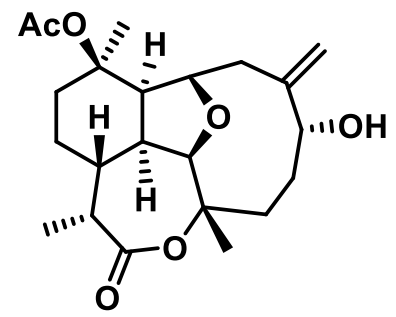

(the best fit to the experimental data still gives unacceptable rmsd $>3.6 \mathrm{ppm}$ )

Rel energy (kcal/mol): $\begin{array}{ccc}\text { Conf } 1 & \text { Conf } 2 \\ 0.0 & 0.9\end{array}$

\begin{tabular}{|c|c|c|c|c|c|c|c|}
\hline C-nom & iGau & Exp & Calc & $\operatorname{diff}$ & 1 & 2 & \\
\hline $\mathrm{C}-\mathrm{C}$ & 11 & 176.10 & 182.51 & 6.41 & [ 182.58 & $182.19]$ & \\
\hline $\mathrm{C}-\mathrm{C}$ & 30 & 169.80 & 170.78 & 0.98 & [ 170.79 & $170.71]$ & \\
\hline $\mathrm{C}-\mathrm{C}$ & 15 & 147.90 & 149.35 & 1.45 & [ 149.21 & $150.01]$ & \\
\hline $\mathrm{C}-\mathrm{C}$ & 13 & 85.20 & 88.52 & 3.32 & {$\left[\begin{array}{l}88.51 \\
0\end{array}\right.$} & $88.55]$ & \\
\hline $\mathrm{C}-\mathrm{C}$ & 6 & 81.00 & 83.51 & 2.51 & 83.37 & $84.17]$ & \\
\hline $\mathrm{C}-\mathrm{CH}$ & 7 & 91.00 & 92.49 & 1.49 & 92.97 & $90.32]$ & \\
\hline $\mathrm{C}-\mathrm{CH}$ & 9 & 82.50 & 82.87 & 0.37 & 82.95 & $82.51]$ & \\
\hline $\mathrm{C}-\mathrm{CH}$ & 16 & 72.60 & 73.91 & 1.31 & 74.32 & $72.03]$ & \\
\hline $\mathrm{C}-\mathrm{CH}$ & 5 & 47.80 & 54.38 & 6.58 & 54.45 & $54.06]$ & \\
\hline $\mathrm{C}-\mathrm{CH}$ & 10 & 45.90 & 47.59 & 1.69 & 47.62 & $47.47]$ & \\
\hline $\mathrm{C}-\mathrm{CH}$ & 4 & 44.90 & 40.90 & -4.00 & 40.62 & $42.18]$ & \\
\hline $\mathrm{C}-\mathrm{CH}$ & 3 & 37.00 & 39.34 & 2.34 & 39.46 & $38.81]$ & \\
\hline $\mathrm{C}-\mathrm{CH} 2$ & 17 & 117.20 & 112.85 & -4.35 & [ 113.52 & $109.82]$ & \\
\hline $\mathrm{C}-\mathrm{CH} 2$ & 14 & 41.30 & 39.96 & -1.34 & 39.10 & $43.88]$ & \\
\hline $\mathrm{C}-\mathrm{CH} 2$ & 1 & 32.70 & 30.95 & -1.75 & 31.31 & $29.29]$ & \\
\hline $\mathrm{C}-\mathrm{CH} 2$ & 19 & 30.40 & 29.63 & -0.77 & 28.67 & $33.99]$ & \\
\hline $\mathrm{C}-\mathrm{CH} 2$ & 18 & 28.70 & 29.24 & 0.54 & 28.61 & $32.13]$ & \\
\hline $\mathrm{C}-\mathrm{CH} 2$ & 2 & 16.80 & 23.19 & 6.39 & 23.23 & $23.00]$ & \\
\hline $\mathrm{C}-\mathrm{CH} 3$ & 25 & 28.90 & 24.17 & -4.73 & 24.21 & $23.97 \mathrm{]}$ & \\
\hline $\mathrm{C}-\mathrm{CH} 3$ & 22 & 22.30 & 23.64 & 1.34 & 23.73 & $23.25]$ & \\
\hline $\mathrm{C}-\mathrm{CH} 3$ & 31 & 20.70 & 22.30 & 1.60 & 22.30 & $22.32]$ & \\
\hline $\mathrm{C}-\mathrm{CH} 3$ & 23 & 17.50 & 9.23 & -8.27 & 9.16 & $9.56]$ & \\
\hline
\end{tabular}

Conformer 1

Energy: -1308.92020 Hartree (Rel: $0.0 \mathrm{kcal} / \mathrm{mol}$ ) XYZ coordinates for conf 1 :

$\begin{array}{lrrr}\text { C } & 3.03059 & -0.50081 & -1.58611 \\ \text { C } & 2.48412 & 0.91364 & -1.89935 \\ \text { C } & 1.57988 & 1.41448 & -0.76578 \\ \text { C } & 0.34370 & 0.49758 & -0.67775 \\ \text { C } & 0.62261 & -0.99611 & -0.99406 \\ \text { C } & 2.08629 & -1.41260 & -0.74818 \\ \text { C } & -0.30990 & 0.47476 & 0.71889 \\ \text { O } & -0.79425 & -0.84681 & 0.93274 \\ \text { C } & -0.47836 & -1.70700 & -0.17253 \\ \text { C } & 1.19540 & 2.92862 & -0.85394 \\ \text { C } & 0.27967 & 3.19435 & 0.33548 \\ \text { O } & -0.99226 & 2.71854 & 0.13851 \\ \text { C } & -1.44635 & 1.52348 & 0.88827 \\ \text { C } & -1.73349 & -1.98511 & -1.03246 \\ \text { C } & -2.98445 & -2.27515 & -0.22382\end{array}$




\begin{tabular}{|c|c|c|c|}
\hline C & -4.02035 & -1.17082 & -0.03066 \\
\hline C & -3.22726 & -3.49686 & 0.26603 \\
\hline C & -3.61754 & 0.03682 & 0.84186 \\
\hline $\mathrm{C}$ & -2.74481 & 1.12727 & 0.16234 \\
\hline $\mathrm{H}$ & 0.45032 & -1.18131 & -2.06074 \\
\hline $\mathrm{H}$ & -0.39429 & 0.84552 & -1.40680 \\
\hline $\mathrm{C}$ & -1.67903 & 1.88828 & 2.35491 \\
\hline C & 0.57862 & 3.36900 & -2.19095 \\
\hline $\mathrm{H}$ & 2.14523 & 1.30618 & 0.16874 \\
\hline $\mathrm{C}$ & 2.32043 & -2.89219 & -1.07677 \\
\hline 0 & 0.63633 & 3.72876 & 1.36344 \\
\hline 0 & -4.45050 & -0.65265 & -1.29856 \\
\hline 0 & 2.27825 & -1.18232 & 0.69639 \\
\hline 0 & 4.43516 & -1.90803 & 0.80421 \\
\hline $\mathrm{C}$ & 3.43529 & -1.46139 & 1.33239 \\
\hline C & 3.31905 & -1.12208 & 2.80168 \\
\hline $\mathrm{H}$ & 3.98362 & -0.43432 & -1.05708 \\
\hline $\mathrm{H}$ & 3.24504 & -1.01744 & -2.52857 \\
\hline $\mathrm{H}$ & 1.91650 & 0.89643 & -2.83821 \\
\hline $\mathrm{H}$ & 3.32516 & 1.59953 & -2.05693 \\
\hline $\mathrm{H}$ & 0.45512 & 0.67343 & 1.48313 \\
\hline $\mathrm{H}$ & -0.13751 & -2.64959 & 0.26717 \\
\hline $\mathrm{H}$ & 2.10764 & 3.50850 & -0.67675 \\
\hline $\mathrm{H}$ & -1.50067 & -2.84245 & -1.67739 \\
\hline $\mathrm{H}$ & -1.92481 & -1.13833 & -1.70033 \\
\hline $\mathrm{H}$ & -4.87643 & -1.63847 & 0.47922 \\
\hline $\mathrm{H}$ & -4.12386 & -3.71630 & 0.84190 \\
\hline $\mathrm{H}$ & -2.53416 & -4.32303 & 0.12007 \\
\hline $\mathrm{H}$ & -4.55350 & 0.50220 & 1.17164 \\
\hline $\mathrm{H}$ & -3.13673 & -0.36286 & 1.73635 \\
\hline $\mathrm{H}$ & -2.50660 & 0.84043 & -0.86441 \\
\hline $\mathrm{H}$ & -3.33084 & 2.04832 & 0.07333 \\
\hline $\mathrm{H}$ & -2.49469 & 2.61354 & 2.44521 \\
\hline $\mathrm{H}$ & -0.77845 & 2.32697 & 2.78947 \\
\hline $\mathrm{H}$ & -1.94096 & 0.99625 & 2.93252 \\
\hline $\mathrm{H}$ & 0.33822 & 4.43756 & -2.16690 \\
\hline $\mathrm{H}$ & 1.28749 & 3.20792 & -3.00913 \\
\hline $\mathrm{H}$ & -0.34325 & 2.82707 & -2.41809 \\
\hline $\mathrm{H}$ & 1.96680 & -3.10058 & -2.09242 \\
\hline $\mathrm{H}$ & 3.38132 & -3.13984 & -1.01867 \\
\hline $\mathrm{H}$ & 1.77639 & -3.54246 & -0.38449 \\
\hline $\mathrm{H}$ & -4.76412 & -1.40544 & -1.82561 \\
\hline $\mathrm{H}$ & 2.42476 & -1.58281 & 3.23153 \\
\hline $\mathrm{H}$ & 3.22001 & -0.03764 & 2.92387 \\
\hline $\mathrm{H}$ & 4.21063 & -1.46583 & 3.32766 \\
\hline
\end{tabular}

$\begin{array}{lrrr}\text { Conformer } 2 & & \\ \text { Energy: -1308.91877 Hartree (Rel: } & 0.9 \mathrm{kcal} / \mathrm{mol}) \\ \text { XYZ coordinates for conf } 2 \text { : } & & \\ \text { C } & 2.91694 & -0.43907 & -1.66151 \\ \text { C } & 2.29876 & 0.95774 & -1.92042 \\ \text { C } & 1.41093 & 1.41539 & -0.75519 \\ \text { C } & 0.23747 & 0.42318 & -0.61062 \\ \text { C } & 0.63433 & -1.07720 & -0.73443 \\ \text { C } & 2.15694 & -1.33152 & -0.64709 \\ \text { C } & -0.48466 & 0.52400 & 0.74732 \\ \text { O } & -0.83180 & -0.78800 & 1.15800 \\ \text { C } & -0.19645 & -1.80105 & 0.36089 \\ \text { C } & 0.94586 & 2.90771 & -0.87275 \\ \text { C } & -0.00257 & 3.16512 & 0.29192 \\ \text { O } & -1.26430 & 2.68294 & 0.05223 \\ \text { C } & -1.73315 & 1.44916 & 0.72458 \\ \text { C } & -1.28380 & -2.77443 & -0.15889\end{array}$




$\begin{array}{rrr}-2.52234 & -2.07052 & -0.66679 \\ -3.45231 & -1.50771 & 0.41343 \\ -2.82137 & -1.99775 & -1.96977 \\ -3.90997 & -0.04986 & 0.20890 \\ -2.88221 & 1.01515 & -0.21591 \\ 0.33239 & -1.44023 & -1.72281 \\ -0.47545 & 0.63279 & -1.41265 \\ -2.21801 & 1.77502 & 2.13500 \\ 0.32684 & 3.29247 & -2.22672 \\ 2.00844 & 1.36510 & 0.16239 \\ 2.49334 & -2.81194 & -0.86850 \\ 0.30858 & 3.71183 & 1.32764 \\ -4.60478 & -2.35547 & 0.57191 \\ 2.48981 & -0.94160 & 0.73914 \\ 4.70300 & -1.47913 & 0.65934 \\ 3.73086 & -1.06104 & 1.25757 \\ 3.74630 & -0.58380 & 2.69263 \\ 3.94711 & -0.34944 & -1.31101 \\ 2.96820 & -0.98982 & -2.60771 \\ 1.69609 & 0.92882 & -2.83650 \\ 3.09973 & 1.68468 & -2.10011 \\ 0.20886 & 0.93706 & 1.49614 \\ 0.47301 & -2.35934 & 1.02355 \\ 1.82868 & 3.53230 & -0.69753 \\ -1.57517 & -3.43294 & 0.67062 \\ -0.84345 & -3.41304 & -0.93354 \\ -2.94247 & -1.58302 & 1.37163 \\ -3.70789 & -1.48238 & -2.33521 \\ -2.19633 & -2.46413 & -2.72773 \\ -4.67707 & -0.05372 & -0.57911 \\ -4.43079 & 0.25729 & 1.12363 \\ -2.45837 & 0.72896 & -1.18247 \\ -3.44574 & 1.93767 & -0.40057 \\ -3.07756 & 2.45255 & 2.10002 \\ -1.42259 & 2.25571 & 2.71071 \\ -2.51690 & 0.85748 & 2.65259 \\ -0.01103 & 4.33448 & -2.21113 \\ 1.06855 & 3.19813 & -3.02556 \\ -0.53507 & 2.66851 & -2.47703 \\ 2.06606 & -3.14544 & -1.82027 \\ 3.57323 & -2.96328 & -0.89871 \\ 2.08085 & -3.43749 & -0.07050 \\ -5.02043 & -2.44001 & -0.30195 \\ 4.73298 & -1.09151 & .49024 \\ -17788 & & \end{array}$


Briarellin $\mathrm{K}$ - revised

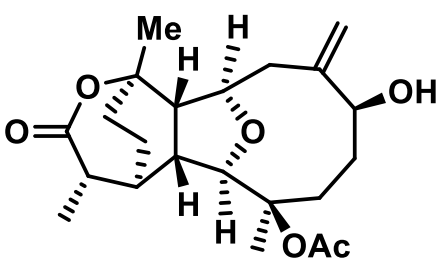

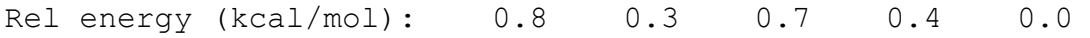

Conf 1 Conf 2 Conf 3 Conf 4 Conf 5

\begin{tabular}{|c|c|c|c|c|c|c|c|c|c|}
\hline C-nom & iGau & Exp & Calc & $\operatorname{diff}$ & 1 & 2 & 3 & 4 & 5 \\
\hline $\mathrm{C} 1-\mathrm{CH}$ & 4 & 44.90 & 45.90 & 1.00 & 45.74 & 45.74 & 45.95 & 45.85 & 46.04 \\
\hline $\mathrm{C} 2-\mathrm{CH}$ & 5 & 91.00 & 91.13 & 0.13 & 95.21 & 92.22 & 95.11 & 91.86 & 87.74 \\
\hline $\mathrm{C} 3-\mathrm{C}$ & 6 & 85.20 & 87.11 & 1.91 & 85.71 & 86.71 & 85.47 & 86.58 & 88.51 \\
\hline $\mathrm{C} 4-\mathrm{CH} 2$ & 10 & 28.70 & 29.26 & 0.56 & 23.53 & 29.56 & 22.51 & 28.69 & 33.05 \\
\hline $\mathrm{C} 5-\mathrm{CH} 2$ & 17 & 32.70 & 29.55 & -3.15 & 26.30 & 26.47 & 27.76 & 27.95 & 33.66 \\
\hline $\mathrm{C} 6-\mathrm{CH}$ & 18 & 72.60 & 73.59 & 0.99 & {$[75.40$} & 75.34 & 74.35 & 74.43 & 71.36 \\
\hline $\mathrm{C} 7-\mathrm{C}$ & 19 & 147.90 & 148.44 & 0.54 & [ 147.96 & 147.71 & 146.90 & 146.90 & 150.27 \\
\hline $\mathrm{C} 8-\mathrm{CH} 2$ & 43 & 41.30 & 40.01 & -1.29 & {$[\quad 38.82$} & 38.54 & 39.11 & 38.95 & 42.05 \\
\hline $\mathrm{C} 9-\mathrm{CH}$ & 15 & 82.50 & 83.59 & 1.09 & 84.13 & 83.89 & 84.10 & 83.82 & 82.98 \\
\hline $\mathrm{ClO}-\mathrm{CH}$ & 14 & 47.80 & 48.47 & 0.67 & 47.37 & 47.98 & 47.53 & 48.23 & 49.48 \\
\hline $\mathrm{C} 11-\mathrm{C}$ & 13 & 81.00 & 81.92 & 0.92 & 82.02 & 81.99 & 82.03 & 82.07 & 81.73 \\
\hline $\mathrm{C} 12-\mathrm{CH} 2$ & 12 & 30.40 & 30.30 & -0.10 & 29.86 & 30.38 & 29.95 & 30.37 & 30.44 \\
\hline $\mathrm{C} 13-\mathrm{CH} 2$ & 9 & 16.80 & 17.14 & 0.34 & 16.70 & 17.21 & 16.86 & 17.24 & 17.25 \\
\hline $\mathrm{C} 14-\mathrm{CH}$ & 3 & 37.00 & 36.94 & -0.06 & 38.35 & 36.64 & 38.43 & 36.75 & 36.36 \\
\hline $\mathrm{C} 15-\mathrm{CH}$ & 2 & 45.90 & 46.77 & 0.87 & {$[\quad 46.69$} & 46.66 & 46.71 & 46.67 & 46.93 \\
\hline C16-C & 8 & 176.10 & 174.59 & -1.51 & [ 174.42 & 174.70 & 174.41 & 174.65 & 174.60 \\
\hline C17-CH3 & 1 & 17.50 & 17.83 & 0.33 & {$[\quad 18.13$} & 17.86 & 18.03 & 17.80 & 17.68 \\
\hline $\mathrm{C} 18-\mathrm{CH} 3$ & 27 & 20.70 & 20.14 & -0.56 & 22.72 & 20.32 & 22.13 & 19.99 & 18.77 \\
\hline $\mathrm{C} 19-\mathrm{CH} 2$ & 42 & 117.20 & 115.38 & -1.82 & 116.31 & 116.17 & 118.68 & 118.30 & 112.13 \\
\hline $\mathrm{C} 20-\mathrm{CH} 3$ & 34 & 28.90 & 28.13 & -0.77 & 27.75 & 28.15 & 27.88 & 28.19 & 28.28 \\
\hline $\mathrm{C} 21-\mathrm{C}$ & 50 & 169.80 & 170.85 & 1.05 & 169.91 & 171.21 & 170.10 & 171.08 & 171.00 \\
\hline \multirow[t]{3}{*}{$\mathrm{C} 22-\mathrm{CH} 3$} & 51 & 22.30 & 21.53 & -0.77 & [ 21.62 & 21.42 & 21.51 & 21.36 & 21.67 \\
\hline & & & em shi & ע. & $1.16 \mathrm{ppm}$ & $(\mathrm{MLAL}=0.9$ & 3) $\mathrm{N}=22$ & $\{-3.15$ & $1.91\}$ \\
\hline & & & & Eracti & $: 0.103$ & 0.223 & 0.114 & 0.189 & 0.371 \\
\hline
\end{tabular}

Conformer 1

Energy: -1308.92839 Hartree (Rel: $0.8 \mathrm{kcal} / \mathrm{mol}$ )

XYZ coordinates for conf 1 :

$\begin{array}{lrrr}\text { C } & 4.01660 & 2.42074 & 0.09998 \\ \text { C } & 2.79854 & 1.56255 & 0.50014 \\ \text { C } & 1.85843 & 1.24790 & -0.68295 \\ \text { C } & 0.62424 & 0.43379 & -0.22438 \\ \text { C } & -0.54016 & 0.53635 & -1.24021 \\ \text { C } & -1.88035 & 1.12213 & -0.69322 \\ \text { O } & 3.06592 & -0.89722 & 1.03831 \\ \text { C } & 3.28466 & 0.39432 & 1.36569 \\ \text { C } & 2.54827 & 0.54145 & -1.85907 \\ \text { C } & -2.57035 & 0.19140 & 0.32549 \\ \text { O } & 3.88392 & 0.61078 & 2.40172 \\ \text { C } & 3.12278 & -0.82413 & -1.43250 \\ \text { C } & 2.43116 & -1.41330 & -0.18872 \\ \text { C } & 0.92391 & -1.08929 & -0.09440 \\ \text { C } & 0.04663 & -1.77859 & -1.16981 \\ \text { O } & -0.76740 & -0.76657 & -1.77350 \\ \text { C } & -3.30106 & -1.01478 & -0.31410\end{array}$




\begin{tabular}{|c|c|c|c|}
\hline $\mathrm{C}$ & -3.11229 & -2.34616 & 0.42344 \\
\hline C & -1.65516 & -2.77551 & 0.58848 \\
\hline $\mathrm{H}$ & 3.68460 & 3.31566 & -0.43746 \\
\hline $\mathrm{H}$ & 4.56415 & 2.73248 & 0.99262 \\
\hline $\mathrm{H}$ & 4.70973 & 1.87347 & -0.54783 \\
\hline $\mathrm{H}$ & 2.21265 & 2.16261 & 1.21030 \\
\hline $\mathrm{H}$ & 1.49373 & 2.22306 & -1.03038 \\
\hline $\mathrm{H}$ & 0.29781 & 0.82695 & 0.74156 \\
\hline $\mathrm{H}$ & -0.24163 & 1.18664 & -2.07435 \\
\hline $\mathrm{C}$ & -2.77835 & 1.51534 & -1.87285 \\
\hline $\mathrm{H}$ & 1.81251 & 0.40120 & -2.65994 \\
\hline $\mathrm{H}$ & 3.33651 & 1.17094 & -2.28364 \\
\hline $\mathrm{H}$ & -1.81972 & -0.15767 & 1.04408 \\
\hline $\mathrm{H}$ & -3.28539 & 0.78853 & 0.89391 \\
\hline $\mathrm{H}$ & 4.19253 & -0.75426 & -1.20358 \\
\hline $\mathrm{H}$ & 3.03868 & -1.54040 & -2.25704 \\
\hline $\mathrm{C}$ & 2.71830 & -2.91253 & -0.07442 \\
\hline 0 & -1.40350 & 2.33715 & -0.00834 \\
\hline $\mathrm{H}$ & 0.60064 & -1.42755 & 0.89224 \\
\hline $\mathrm{H}$ & 0.67292 & -2.19044 & -1.97347 \\
\hline $\mathrm{H}$ & 2.30863 & -3.31686 & 0.85632 \\
\hline $\mathrm{H}$ & -4.37889 & -0.82308 & -0.35611 \\
\hline $\mathrm{H}$ & -2.96489 & -1.16763 & -1.33872 \\
\hline 0 & -3.80754 & -2.24379 & 1.66299 \\
\hline $\mathrm{C}$ & -1.15408 & -3.04866 & 1.80034 \\
\hline $\mathrm{C}$ & -0.82379 & -2.96923 & -0.66992 \\
\hline $\mathrm{H}$ & -0.14650 & -3.43515 & 1.92970 \\
\hline $\mathrm{H}$ & -1.73165 & -2.89156 & 2.70566 \\
\hline $\mathrm{H}$ & -0.13463 & -3.80626 & -0.50743 \\
\hline $\mathrm{H}$ & -1.46817 & -3.26011 & -1.50967 \\
\hline $\mathrm{H}$ & 2.28907 & -3.46152 & -0.91752 \\
\hline $\mathrm{H}$ & 3.79971 & -3.08330 & -0.07352 \\
\hline $\mathrm{C}$ & -2.24343 & 3.24405 & 0.53450 \\
\hline C & -1.45540 & 4.36358 & 1.17909 \\
\hline 0 & -3.45620 & 3.17498 & 0.51936 \\
\hline $\mathrm{H}$ & -2.14141 & 5.12534 & 1.55140 \\
\hline $\mathrm{H}$ & -0.86029 & 3.96927 & 2.00993 \\
\hline $\mathrm{H}$ & -0.76065 & 4.80598 & 0.45832 \\
\hline $\mathrm{H}$ & -3.76881 & 1.82659 & -1.53841 \\
\hline $\mathrm{H}$ & -2.31863 & 2.34229 & -2.42521 \\
\hline $\mathrm{H}$ & -2.88132 & 0.67176 & -2.56085 \\
\hline $\mathrm{H}$ & -3.59451 & -3.11940 & -0.20206 \\
\hline $\mathrm{H}$ & -3.79289 & -3.11878 & 2.08321 \\
\hline
\end{tabular}

Conformer 2

Energy: -1308.92911 Hartree (Rel: $0.3 \mathrm{kcal} / \mathrm{mol})$ XYZ coordinates for conf 2 :

$\begin{array}{lrrr}C & 4.51713 & 0.87211 & 0.09242 \\ C & 3.07952 & 0.52009 & 0.52713 \\ C & 2.04758 & 0.63732 & -0.61495 \\ C & 0.62128 & 0.28278 & -0.12165 \\ C & -0.47483 & 0.88381 & -1.03652 \\ C & -1.55463 & 1.77066 & -0.32984 \\ O & 2.43760 & -1.89008 & 0.96203 \\ C & 3.11409 & -0.78209 & 1.33568 \\ C & 2.39648 & -0.19593 & -1.85590 \\ C & -2.52134 & 0.95180 & 0.55136 \\ O & 3.75913 & -0.84569 & 2.36497 \\ C & 2.49686 & -1.69185 & -1.50488 \\ C & 1.65483 & -2.07899 & -0.27306 \\ C & 0.35700 & -1.25399 & -0.12222 \\ C & -0.68418 & -1.49246 & -1.24442 \\ O & -1.12983 & -0.20744 & -1.69268\end{array}$




\begin{tabular}{|c|c|c|c|}
\hline C & -3.61054 & 0.16839 & -0.21671 \\
\hline C & -3.88465 & -1.23751 & 0.33250 \\
\hline $\mathrm{C}$ & -2.64512 & -2.12537 & 0.41083 \\
\hline $\mathrm{H}$ & 4.53048 & 1.84918 & -0.40267 \\
\hline $\mathrm{H}$ & 5.17232 & 0.91416 & 0.96601 \\
\hline $\mathrm{H}$ & 4.92886 & 0.13339 & -0.60348 \\
\hline $\mathrm{H}$ & 2.79177 & 1.26451 & 1.28239 \\
\hline $\mathrm{H}$ & 2.04377 & 1.69553 & -0.89864 \\
\hline $\mathrm{H}$ & 0.50080 & 0.67118 & 0.89397 \\
\hline $\mathrm{H}$ & -0.01431 & 1.49991 & -1.81394 \\
\hline $\mathrm{C}$ & -2.30437 & 2.63840 & -1.34600 \\
\hline $\mathrm{H}$ & 1.61579 & -0.03919 & -2.61005 \\
\hline $\mathrm{H}$ & 3.32877 & 0.15244 & $-2 \cdot 31173$ \\
\hline $\mathrm{H}$ & -1.93323 & 0.27005 & 1.17647 \\
\hline $\mathrm{H}$ & -2.99183 & 1.65742 & 1.24312 \\
\hline $\mathrm{H}$ & 3.53208 & -1.98300 & -1.29145 \\
\hline $\mathrm{H}$ & 2.18387 & -2.30249 & -2.35865 \\
\hline C & 1.40288 & -3.58814 & -0.25059 \\
\hline O & -0.91424 & 2.65745 & 0.66629 \\
\hline $\mathrm{H}$ & -0.08308 & -1.53875 & 0.83635 \\
\hline $\mathrm{H}$ & -0.20871 & -1.97571 & -2.10990 \\
\hline $\mathrm{H}$ & 0.88871 & -3.88408 & 0.66902 \\
\hline $\mathrm{H}$ & -4.56055 & 0.71331 & -0.19195 \\
\hline $\mathrm{H}$ & -3.33337 & 0.05611 & -1.26391 \\
\hline O & -4.53899 & -1.07863 & 1.58851 \\
\hline C & -2.27954 & -2.69560 & 1.56621 \\
\hline C & -1.89279 & -2.40406 & -0.87940 \\
\hline $\mathrm{H}$ & -1.44545 & -3.39005 & 1.62305 \\
\hline $\mathrm{H}$ & -2.79647 & -2.48333 & 2.49665 \\
\hline $\mathrm{H}$ & -1.50691 & -3.42911 & -0.84268 \\
\hline $\mathrm{H}$ & -2.58021 & -2.36639 & -1.73450 \\
\hline $\mathrm{H}$ & 0.80012 & -3.89839 & -1.10897 \\
\hline $\mathrm{H}$ & 2.35744 & -4.12247 & -0.29515 \\
\hline C & 0.01706 & 3.57309 & 0.33967 \\
\hline C & 0.36182 & 4.42498 & 1.54101 \\
\hline O & 0.51775 & 3.70221 & -0.76268 \\
\hline $\mathrm{H}$ & 1.17745 & 5.10295 & 1.28703 \\
\hline $\mathrm{H}$ & -0.51555 & 5.00411 & 1.84848 \\
\hline $\mathrm{H}$ & 0.64868 & 3.79353 & 2.38767 \\
\hline $\mathrm{H}$ & -3.08254 & 3.22469 & -0.84535 \\
\hline $\mathrm{H}$ & -1.61906 & 3.31886 & -1.85483 \\
\hline $\mathrm{H}$ & -2.78075 & 2.00789 & -2.10156 \\
\hline $\mathrm{H}$ & -4.57565 & -1.72429 & -0.37938 \\
\hline $\mathrm{H}$ & -4.81196 & -1.95908 & 1.89318 \\
\hline
\end{tabular}

Conformer 3

Energy: -1308.92848 Hartree (Rel: $0.7 \mathrm{kcal} / \mathrm{mol}$ ) XYZ coordinates for conf 3 :

$\begin{array}{lrrr}C & 4.00928 & 2.45576 & 0.09385 \\ C & 2.78396 & 1.60810 & 0.49423 \\ C & 1.85346 & 1.27995 & -0.69292 \\ C & 0.61919 & 0.46505 & -0.23511 \\ C & -0.54384 & 0.56217 & -1.25333 \\ C & -1.89733 & 1.10285 & -0.69625 \\ \text { O } & 3.04148 & -0.84502 & 1.06509 \\ C & 3.25522 & 0.45010 & 1.38130 \\ C & 2.55393 & 0.56414 & -1.85667 \\ C & -2.54405 & 0.13996 & 0.32049 \\ O & 3.83590 & 0.67885 & 2.42524 \\ C & 3.13828 & -0.78940 & -1.40654 \\ C & 2.43145 & -1.37416 & -0.16864 \\ C & 0.92072 & -1.05794 & -0.10262 \\ C & 0.07141 & -1.74511 & -1.20132\end{array}$




\begin{tabular}{|c|c|c|c|}
\hline 0 & -0.74133 & -0.73434 & -1.81107 \\
\hline C & -3.25628 & -1.08073 & -0.31559 \\
\hline C & -3.05562 & -2.39171 & 0.46206 \\
\hline C & -1.59939 & -2.85521 & 0.54781 \\
\hline $\mathrm{H}$ & 3.68605 & 3.34492 & -0.45832 \\
\hline $\mathrm{H}$ & 4.54983 & 2.77709 & 0.98739 \\
\hline $\mathrm{H}$ & 4.70606 & 1.89727 & -0.54026 \\
\hline $\mathrm{H}$ & 2.19406 & 2.22069 & 1.19013 \\
\hline $\mathrm{H}$ & 1.48815 & 2.25071 & -1.05193 \\
\hline $\mathrm{H}$ & 0.29120 & 0.86119 & 0.72929 \\
\hline $\mathrm{H}$ & -0.25967 & 1.23552 & -2.07384 \\
\hline $\mathrm{C}$ & -2.81876 & 1.47149 & -1.86500 \\
\hline $\mathrm{H}$ & 1.82234 & 0.40553 & -2.65801 \\
\hline $\mathrm{H}$ & 3.33867 & 1.19419 & -2.28685 \\
\hline $\mathrm{H}$ & -1.76151 & -0.19396 & 1.01518 \\
\hline $\mathrm{H}$ & -3.26128 & 0.71333 & 0.91119 \\
\hline $\mathrm{H}$ & 4.20287 & -0.70366 & -1.15968 \\
\hline $\mathrm{H}$ & 3.07794 & -1.51491 & -2.22494 \\
\hline $\mathrm{C}$ & 2.72549 & -2.87063 & -0.03960 \\
\hline O & -1.45639 & 2.32936 & -0.00964 \\
\hline $\mathrm{H}$ & 0.57748 & -1.40511 & 0.87496 \\
\hline $\mathrm{H}$ & 0.71773 & -2.13743 & -1.99865 \\
\hline $\mathrm{H}$ & 2.30210 & -3.27118 & 0.88649 \\
\hline $\mathrm{H}$ & -4.33482 & -0.89635 & -0.36912 \\
\hline $\mathrm{H}$ & -2.90812 & -1.23999 & -1.33571 \\
\hline 0 & -3.69243 & -2.34039 & 1.73809 \\
\hline C & -1.08907 & -3.26478 & 1.71639 \\
\hline C & -0.78887 & -2.95666 & -0.73706 \\
\hline $\mathrm{H}$ & -0.07940 & -3.66076 & 1.79143 \\
\hline $\mathrm{H}$ & -1.67635 & -3.24674 & 2.62836 \\
\hline $\mathrm{H}$ & -0.09365 & -3.79719 & -0.63027 \\
\hline $\mathrm{H}$ & -1.44605 & -3.21142 & -1.57870 \\
\hline $\mathrm{H}$ & 2.31380 & -3.42734 & -0.88646 \\
\hline $\mathrm{H}$ & 3.80772 & -3.03475 & -0.01943 \\
\hline $\mathrm{C}$ & -2.32343 & 3.20149 & 0.54851 \\
\hline C & -1.57340 & 4.36831 & 1.15232 \\
\hline O & -3.53132 & 3.07243 & 0.56868 \\
\hline $\mathrm{H}$ & -2.28217 & 5.06340 & 1.60379 \\
\hline $\mathrm{H}$ & -0.86927 & 4.01229 & 1.91159 \\
\hline $\mathrm{H}$ & -0.99121 & 4.88199 & 0.38027 \\
\hline $\mathrm{H}$ & -3.81315 & 1.75642 & -1.51867 \\
\hline $\mathrm{H}$ & -2.38678 & 2.31042 & -2.42149 \\
\hline $\mathrm{H}$ & -2.90734 & 0.62534 & -2.55150 \\
\hline $\mathrm{H}$ & -3.60687 & -3.17379 & -0.07940 \\
\hline $\mathrm{H}$ & -3.27988 & -1.62092 & 2.24415 \\
\hline \multicolumn{4}{|c|}{ Conformer 4} \\
\hline & 308.92896 & Hartree (Rel: & $0.4 \mathrm{kcal} / \mathrm{mol}$ ) \\
\hline \multicolumn{4}{|c|}{ XYZ coordinates for conf 4 : } \\
\hline $\mathrm{C}$ & 4.51807 & 0.85624 & 0.11467 \\
\hline $\mathrm{C}$ & 3.07473 & 0.51583 & 0.53937 \\
\hline $\mathrm{C}$ & 2.05133 & 0.64120 & -0.60940 \\
\hline $\mathrm{C}$ & 0.61994 & 0.29360 & -0.12623 \\
\hline $\mathrm{C}$ & -0.46604 & 0.90638 & -1.04506 \\
\hline C & -1.55352 & 1.77701 & -0.33425 \\
\hline O & 2.41015 & -1.88912 & 0.96813 \\
\hline $\mathrm{C}$ & 3.09167 & -0.78648 & 1.34820 \\
\hline $\mathrm{C}$ & 2.40371 & -0.19174 & -1.84938 \\
\hline $\mathrm{C}$ & -2.50084 & 0.93646 & 0.54719 \\
\hline 0 & 3.72563 & -0.85442 & 2.38406 \\
\hline $\mathrm{C}$ & 2.49396 & -1.68833 & -1.49864 \\
\hline C & 1.63884 & -2.07316 & -0.27500 \\
\hline C & 0.34372 & -1.24150 & -0.13606 \\
\hline
\end{tabular}




\begin{tabular}{|c|c|c|c|}
\hline $\mathrm{C}$ & -0.68411 & -1.46621 & -1.27360 \\
\hline O & -1.11422 & -0.17494 & -1.72150 \\
\hline $\mathrm{C}$ & -3.59206 & 0.14265 & -0.20924 \\
\hline C & -3.86234 & -1.24668 & 0.39175 \\
\hline $\mathrm{C}$ & -2.64963 & -2.17876 & 0.37455 \\
\hline $\mathrm{H}$ & 4.54255 & 1.83299 & -0.38062 \\
\hline $\mathrm{H}$ & 5.16732 & 0.89357 & 0.99291 \\
\hline $\mathrm{H}$ & 4.92893 & 0.11409 & -0.57802 \\
\hline $\mathrm{H}$ & 2.78785 & 1.26266 & 1.29252 \\
\hline $\mathrm{H}$ & 2.05501 & 1.70019 & -0.89089 \\
\hline $\mathrm{H}$ & 0.49711 & 0.67919 & 0.89034 \\
\hline $\mathrm{H}$ & 0.00042 & 1.53526 & -1.80814 \\
\hline $\mathrm{C}$ & -2.32266 & 2.63470 & -1.34345 \\
\hline $\mathrm{H}$ & 1.62889 & -0.02985 & -2.60849 \\
\hline $\mathrm{H}$ & 3.34090 & 0.15213 & -2.29848 \\
\hline $\mathrm{H}$ & -1.88764 & 0.25773 & 1.15502 \\
\hline $\mathrm{H}$ & -2.96886 & 1.63086 & 1.25286 \\
\hline $\mathrm{H}$ & 3.52583 & -1.98427 & -1.27589 \\
\hline $\mathrm{H}$ & 2.18626 & -2.29692 & -2.35574 \\
\hline $\mathrm{C}$ & 1.38018 & -3.58109 & -0.25714 \\
\hline 0 & -0.92326 & 2.66876 & 0.66299 \\
\hline $\mathrm{H}$ & -0.11220 & -1.53069 & 0.81458 \\
\hline $\mathrm{H}$ & -0.19953 & -1.94481 & -2.13631 \\
\hline $\mathrm{H}$ & 0.86020 & -3.87634 & 0.65925 \\
\hline $\mathrm{H}$ & -4.53828 & 0.69418 & -0.19275 \\
\hline $\mathrm{H}$ & -3.31419 & 0.01180 & -1.25460 \\
\hline O & -4.45717 & -1.14233 & 1.68515 \\
\hline C & -2.32360 & -2.88374 & 1.46558 \\
\hline C & -1.90115 & -2.37564 & -0.93558 \\
\hline $\mathrm{H}$ & -1.50541 & -3.59934 & 1.45938 \\
\hline $\mathrm{H}$ & -2.88461 & -2.79147 & 2.38953 \\
\hline $\mathrm{H}$ & -1.52716 & -3.40533 & -0.95633 \\
\hline $\mathrm{H}$ & -2.59516 & -2.28973 & -1.78194 \\
\hline $\mathrm{H}$ & 0.78049 & -3.88699 & -1.11924 \\
\hline $\mathrm{H}$ & 2.33266 & -4.11941 & -0.29798 \\
\hline C & -0.00018 & 3.59424 & 0.33598 \\
\hline C & 0.33494 & 4.45038 & 1.53671 \\
\hline 0 & 0.49803 & 3.72766 & -0.76650 \\
\hline $\mathrm{H}$ & 1.15214 & 5.12775 & 1.28630 \\
\hline $\mathrm{H}$ & -0.54503 & 5.03119 & 1.83368 \\
\hline $\mathrm{H}$ & 0.61478 & 3.82304 & 2.38865 \\
\hline $\mathrm{H}$ & -3.10712 & 3.20793 & -0.83759 \\
\hline $\mathrm{H}$ & -1.64992 & 3.32699 & -1.85314 \\
\hline $\mathrm{H}$ & -2.79232 & 1.99833 & -2.09813 \\
\hline $\mathrm{H}$ & -4.64090 & -1.72050 & -0.22272 \\
\hline $\mathrm{H}$ & -3.81624 & -0.70141 & 2.26741 \\
\hline
\end{tabular}

Conformer 5

Energy: -1308.92959 Hartree (Rel: $0.0 \mathrm{kcal} / \mathrm{mol}$ ) XYZ coordinates for conf 5:

$\begin{array}{lrrr}C & 4.55699 & 0.77373 & -0.07111 \\ C & 3.14329 & 0.44524 & 0.45145 \\ C & 2.04522 & 0.55477 & -0.62871 \\ C & 0.65290 & 0.21552 & -0.03826 \\ C & -0.50952 & 0.83155 & -0.85650 \\ C & -1.47724 & 1.75056 & -0.05193 \\ \text { O } & 2.48800 & -1.94635 & 0.97056 \\ C & 3.20746 & -0.84491 & 1.27855 \\ C & 2.31832 & -0.30079 & -1.87336 \\ C & -2.18207 & 1.04028 & 1.13614 \\ \text { O } & 3.91345 & -0.90112 & 2.26730 \\ C & 2.43044 & -1.79087 & -1.50080 \\ C & 1.64733 & -2.15450 & -0.22290\end{array}$




\begin{tabular}{|c|c|c|}
\hline 0.36482 & -1.31644 & -0.02960 \\
\hline-0.71215 & -1.52925 & -1.12177 \\
\hline-1.27293 & -0.24275 & -1.40792 \\
\hline-3.56921 & 0.39633 & 0.94274 \\
\hline-3.70380 & -0.84252 & 0.03967 \\
\hline-2.73937 & -1.98207 & 0.38007 \\
\hline 4.55296 & 1.74403 & -0.57954 \\
\hline 5.26187 & 0.81933 & 0.76261 \\
\hline 4.91974 & 0.02134 & -0.77936 \\
\hline 2.90889 & 1.20383 & 1.21117 \\
\hline 2.03179 & 1.60821 & -0.93266 \\
\hline 0.61927 & 0.60120 & 0.98508 \\
\hline-0.11390 & 1.42531 & -1.68561 \\
\hline-2.46590 & 2.46302 & -0.97671 \\
\hline 1.49932 & -0.15078 & -2.58737 \\
\hline 3.22614 & 0.03333 & -2.38524 \\
\hline-1.50134 & 0.30341 & 1.57556 \\
\hline-2.30913 & 1.81760 & 1.89729 \\
\hline 3.47393 & -2.08241 & -1.33344 \\
\hline 2.07475 & -2.41454 & -2.32790 \\
\hline 1.38951 & -3.66121 & -0.16190 \\
\hline-0.66344 & 2.76683 & 0.66154 \\
\hline-0.05882 & -1.59895 & 0.93963 \\
\hline-0.25071 & -1.91216 & -2.04369 \\
\hline 0.89271 & -3.93368 & 0.77460 \\
\hline-4.28912 & 1.14015 & 0.58292 \\
\hline-3.91331 & 0.11031 & 1.94690 \\
\hline-5.06655 & -1.30172 & 0.05492 \\
\hline-2.70927 & -2.53300 & 1.60027 \\
\hline-1.86597 & -2.48696 & -0.74542 \\
\hline-2.07032 & -3.38237 & 1.82926 \\
\hline-3.32740 & -2.17106 & 2.42033 \\
\hline-1.44124 & -3.46429 & -0.49419 \\
\hline-2.48381 & -2.62685 & -1.64271 \\
\hline 0.76755 & -3.98772 & -1.00027 \\
\hline 2.34105 & -4.19996 & -0.21520 \\
\hline 0.11539 & 3.67286 & 0.03777 \\
\hline 0.69120 & 4.64556 & 1.04366 \\
\hline 0.33309 & 3.71447 & -1.15892 \\
\hline 1.40098 & 5.30768 & 0.54643 \\
\hline-0.11538 & 5.23946 & 1.48702 \\
\hline 1.18596 & 4.10741 & 1.85803 \\
\hline-3.12575 & 3.11973 & -0.39973 \\
\hline-1.93646 & 3.06151 & -1.72002 \\
\hline-3.08248 & 1.72910 & -1.50193 \\
\hline-3.53702 & -0.55129 & -0.99626 \\
\hline-5.22384 & -1.71322 & 0.92032 \\
\hline
\end{tabular}


Briarellin L - original

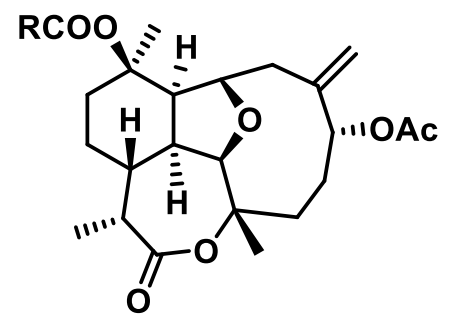

$$
\text { Rel energy (kcal/mol): } \begin{array}{cc}
\text { Conf } 1 & \text { Conf } 2 \\
0.1 & 0.0
\end{array}
$$

\begin{tabular}{|c|c|c|c|c|c|c|}
\hline C-nom & iGau & Exp & Calc & $\operatorname{diff}$ & 1 & 2 \\
\hline$C-C$ & 11 & 176.20 & 182.12 & 5.92 & [ 182.25 & 182.02 \\
\hline $\mathrm{C}-\mathrm{C}$ & 57 & 172.40 & 170.29 & -2.11 & 169.65 & 170.82 \\
\hline $\mathrm{C}-\mathrm{C}$ & 30 & 170.70 & 170.71 & 0.01 & 170.66 & 170.76 \\
\hline $\mathrm{C}-\mathrm{C}$ & 15 & 144.50 & 145.17 & 0.67 & 146.68 & 143.92 \\
\hline $\mathrm{C}-\mathrm{C}$ & 13 & 84.80 & 88.11 & 3.31 & 88.14 & 88.09 \\
\hline$C-C$ & 6 & 80.90 & 83.78 & 2.88 & 83.31 & 84.18 \\
\hline $\mathrm{C}-\mathrm{CH}$ & 7 & 90.60 & 91.42 & 0.82 & 92.88 & 90.20 \\
\hline $\mathrm{C}-\mathrm{CH}$ & 9 & 82.20 & 82.60 & 0.40 & 82.91 & 82.34 \\
\hline $\mathrm{C}-\mathrm{CH}$ & 16 & 74.90 & 77.51 & 2.61 & 78.96 & 76.30 \\
\hline $\mathrm{C}-\mathrm{CH}$ & 5 & 48.00 & 54.12 & 6.12 & 54.21 & 54.05 \\
\hline $\mathrm{C}-\mathrm{CH}$ & 10 & 45.80 & 47.37 & 1.57 & 47.47 & 47.29 \\
\hline $\mathrm{C}-\mathrm{CH}$ & 4 & 44.90 & 41.44 & -3.46 & 40.53 & 42.19 \\
\hline $\mathrm{C}-\mathrm{CH}$ & 3 & 36.90 & 38.97 & 2.07 & 39.30 & 38.69 \\
\hline $\mathrm{C}-\mathrm{CH} 2$ & 17 & 118.60 & 117.57 & -1.03 & 117.30 & 117.80 \\
\hline $\mathrm{C}-\mathrm{CH} 2$ & 14 & 41.50 & 42.28 & 0.78 & 40.45 & 43.81 \\
\hline $\mathrm{C}-\mathrm{CH} 2$ & 19 & 30.30 & 31.45 & 1.15 & 29.27 & 33.26 \\
\hline $\mathrm{C}-\mathrm{CH} 2$ & 1 & 30.10 & 30.14 & 0.04 & 31.23 & 29.23 \\
\hline $\mathrm{C}-\mathrm{CH} 2$ & 18 & 28.30 & 27.84 & -0.46 & 27.07 & 28.48 \\
\hline $\mathrm{C}-\mathrm{CH} 2$ & 2 & 16.80 & 23.16 & 6.36 & 23.32 & 23.03 \\
\hline $\mathrm{C}-\mathrm{CH} 3$ & 25 & 28.90 & 23.94 & -4.96 & 23.97 & 23.92 \\
\hline $\mathrm{C}-\mathrm{CH} 3$ & 22 & 21.30 & 23.34 & 2.04 & 23.67 & 23.07 \\
\hline $\mathrm{C}-\mathrm{CH} 3$ & 31 & 21.00 & 22.34 & 1.34 & 22.30 & 22.38 \\
\hline $\mathrm{C}-\mathrm{CH} 3$ & 61 & 20.50 & 20.57 & 0.07 & 20.49 & 20.63 \\
\hline $\mathrm{C}-\mathrm{CH} 3$ & 23 & 17.40 & 9.43 & -7.97 & 9.19 & 9.63 \\
\hline
\end{tabular}

13C chem shifts: $\mathrm{RMSD}=3.30 \mathrm{ppm}(\mathrm{MAE}=2.42) \mathrm{N}=24 \quad\left\{\begin{array}{lll}-7.97 & 6.36\end{array}\right\}$

Fractions: 0.4540 .546

$\begin{array}{lrrr}\text { Conformer } 1 \\ \text { Energy: -1461.58786 } & \text { Hartree (Rel: } 0.1 \mathrm{kcal} / \mathrm{mol} \text { ) } \\ \text { XYZ coordinates for conf } 1 \text { : } & \\ \text { X } & 3.38324 & -0.53770 & -1.88118 \\ \text { C } & 2.82337 & 0.88711 & -2.11543 \\ \text { C } & 2.08427 & 1.39733 & -0.87100 \\ \text { C } & 0.85778 & 0.49775 & -0.62140 \\ \text { C } & 1.06805 & -0.99710 & -0.97888 \\ \text { C } & 2.54405 & -1.43859 & -0.92739 \\ \text { C } & 0.39406 & 0.47598 & 0.84927 \\ \text { C } & -0.08214 & -0.83963 & 1.11721 \\ \text { O } & 0.06914 & -1.69501 & -0.02716 \\ \text { C } & 1.71146 & 2.91632 & -0.90734 \\ \text { C } & 0.97298 & 3.19028 & 0.39746 \\ \text { C } & -0.32273 & 2.73574 & 0.37562 \\ \text { O } & -0.68945 & 1.54438 & 1.17502 \\ \text { C } & -1.29274 & -1.93838 & -0.72047 \\ \text { C } & -2.43362 & -2.21781 & 0.24067 \\ \text { C } & & & \end{array}$




\begin{tabular}{|c|c|c|c|}
\hline C & -3.40565 & -1.10798 & 0.60194 \\
\hline C & -2.61590 & -3.43310 & 0.76884 \\
\hline $\mathrm{C}$ & -2.86540 & 0.08745 & 1.41488 \\
\hline $\mathrm{C}$ & -2.08165 & 1.17511 & 0.62994 \\
\hline $\mathrm{H}$ & 0.75695 & -1.17048 & -2.01574 \\
\hline $\mathrm{H}$ & 0.03498 & 0.86136 & -1.24452 \\
\hline C & -0.71815 & 1.90271 & 2.66115 \\
\hline $\mathrm{C}$ & 0.92120 & 3.36472 & -2.14711 \\
\hline $\mathrm{H}$ & 2.76748 & 1.28006 & -0.01996 \\
\hline C & 2.70983 & -2.92030 & -1.28361 \\
\hline O & 1.47538 & 3.71118 & 1.36956 \\
\hline 0 & -3.98406 & -0.63464 & -0.65785 \\
\hline 0 & 2.92881 & -1.21600 & 0.47997 \\
\hline 0 & 5.07152 & -1.96861 & 0.29661 \\
\hline $\mathrm{C}$ & 4.15748 & -1.51128 & 0.95495 \\
\hline $\mathrm{C}$ & 4.24565 & -1.17293 & 2.42631 \\
\hline $\mathrm{H}$ & 4.39939 & -0.48894 & -1.48404 \\
\hline $\mathrm{H}$ & 3.46132 & -1.05343 & -2.84509 \\
\hline $\mathrm{H}$ & 2.13709 & 0.88341 & -2.97174 \\
\hline $\mathrm{H}$ & 3.64758 & 1.56007 & -2.38081 \\
\hline $\mathrm{H}$ & 1.25595 & 0.65545 & 1.50718 \\
\hline $\mathrm{H}$ & 0.44301 & -2.64893 & 0.35737 \\
\hline $\mathrm{H}$ & 2.64676 & 3.48433 & -0.85903 \\
\hline $\mathrm{H}$ & -1.16531 & -2.79329 & -1.39662 \\
\hline $\mathrm{H}$ & -1.54459 & -1.08131 & -1.35380 \\
\hline $\mathrm{H}$ & -4.21994 & -1.54826 & 1.18075 \\
\hline $\mathrm{H}$ & -3.42924 & -3.64561 & 1.45839 \\
\hline $\mathrm{H}$ & -1.95002 & -4.26195 & 0.53776 \\
\hline $\mathrm{H}$ & -3.73433 & 0.55548 & 1.88869 \\
\hline $\mathrm{H}$ & -2.26065 & -0.33647 & 2.21833 \\
\hline $\mathrm{H}$ & -1.98626 & 0.90010 & -0.42372 \\
\hline $\mathrm{H}$ & -2.66139 & 2.10459 & 0.63282 \\
\hline $\mathrm{H}$ & -1.50012 & 2.64264 & 2.86210 \\
\hline $\mathrm{H}$ & 0.24048 & 2.32166 & 2.97375 \\
\hline $\mathrm{H}$ & -0.91830 & 1.01199 & 3.26523 \\
\hline $\mathrm{H}$ & 0.69400 & 4.43482 & -2.08889 \\
\hline $\mathrm{H}$ & 1.51086 & 3.20074 & -3.05439 \\
\hline $\mathrm{H}$ & -0.02664 & 2.82942 & -2.24730 \\
\hline $\mathrm{H}$ & 2.23398 & -3.12027 & -2.24970 \\
\hline $\mathrm{H}$ & 3.76570 & -3.18531 & -1.35299 \\
\hline $\mathrm{H}$ & 2.24196 & -3.56302 & -0.53123 \\
\hline C & -5.28052 & -0.25456 & -0.64778 \\
\hline $\mathrm{H}$ & 3.40232 & -1.60602 & 2.97195 \\
\hline $\mathrm{H}$ & 4.19930 & -0.08596 & 2.55820 \\
\hline $\mathrm{H}$ & 5.18803 & -1.54521 & 2.83012 \\
\hline $\mathrm{C}$ & -5.72116 & 0.18371 & -2.02478 \\
\hline 0 & -5.98646 & -0.26965 & 0.34121 \\
\hline $\mathrm{H}$ & -5.11149 & 1.02812 & -2.36288 \\
\hline $\mathrm{H}$ & -5.57981 & -0.63105 & -2.74230 \\
\hline $\mathrm{H}$ & -6.77159 & 0.47546 & -1.99796 \\
\hline
\end{tabular}

Conformer 2 XYZ coordinates for conf 2 :

$\begin{array}{lrrr}\text { C } & 3.48348 & -1.05655 & -1.45709 \\ \text { C } & 3.33342 & 0.47566 & -1.62859 \\ \text { C } & 2.45829 & 1.09106 & -0.52799 \\ \text { C } & 1.04195 & 0.48721 & -0.62861 \\ \text { C } & 1.00492 & -1.04980 & -0.87538 \\ \text { C } & 2.36184 & -1.75362 & -0.64540 \\ \text { C } & 0.19612 & 0.69699 & 0.64283 \\ \text { O } & -0.58177 & -0.47046 & 0.84675 \\ C & -0.14345 & -1.57058 & 0.03281\end{array}$




\begin{tabular}{|c|c|c|c|}
\hline C & 2.46231 & 2.65877 & -0.53129 \\
\hline C & 1.47161 & 3.10291 & 0.53778 \\
\hline 0 & 0.17142 & 3.03504 & 0.10319 \\
\hline C & -0.71505 & 1.95226 & 0.58567 \\
\hline $\mathrm{C}$ & -1.37642 & -2.14625 & -0.70542 \\
\hline C & -2.30538 & -1.08845 & -1.26370 \\
\hline $\mathrm{C}$ & -3.14339 & -0.33925 & -0.23273 \\
\hline C & -2.42887 & -0.85938 & -2.57541 \\
\hline C & -3.13531 & 1.19802 & -0.31019 \\
\hline C & -1.79916 & 1.93408 & -0.51620 \\
\hline $\mathrm{H}$ & 0.75340 & -1.23218 & -1.92600 \\
\hline $\mathrm{H}$ & 0.53720 & 0.95777 & -1.47661 \\
\hline C & -1.27668 & 2.30625 & 1.96039 \\
\hline C & 2.17752 & 3.30721 & -1.89624 \\
\hline $\mathrm{H}$ & 2.87957 & 0.79678 & 0.44008 \\
\hline C & 2.28706 & -3.24567 & -0.99399 \\
\hline 0 & 1.77474 & 3.45579 & 1.65652 \\
\hline 0 & -4.53392 & -0.77186 & -0.43875 \\
\hline O & 2.59050 & -1.58728 & 0.80553 \\
\hline O & 4.54682 & -2.75034 & 0.92043 \\
\hline C & 3.65774 & -2.10993 & 1.44673 \\
\hline C & 3.59828 & -1.77198 & 2.91966 \\
\hline $\mathrm{H}$ & 4.43417 & -1.30287 & -0.97987 \\
\hline $\mathrm{H}$ & 3.51234 & -1.52723 & -2.44649 \\
\hline $\mathrm{H}$ & 2.88344 & 0.69404 & -2.60494 \\
\hline $\mathrm{H}$ & 4.32576 & 0.94213 & -1.63436 \\
\hline $\mathrm{H}$ & 0.86522 & 0.81589 & 1.50909 \\
\hline $\mathrm{H}$ & 0.24260 & -2.34126 & 0.70808 \\
\hline $\mathrm{H}$ & 3.45296 & 2.97675 & -0.18821 \\
\hline $\mathrm{H}$ & -1.94149 & -2.75952 & 0.01007 \\
\hline $\mathrm{H}$ & -1.03380 & -2.82170 & -1.49752 \\
\hline $\mathrm{H}$ & -2.86243 & -0.65714 & 0.76642 \\
\hline $\mathrm{H}$ & -3.11419 & -0.11273 & -2.96907 \\
\hline $\mathrm{H}$ & -1.86245 & -1.42837 & -3.30884 \\
\hline $\mathrm{H}$ & -3.76890 & 1.47932 & -1.16101 \\
\hline $\mathrm{H}$ & -3.64493 & 1.57148 & 0.58504 \\
\hline $\mathrm{H}$ & -1.34897 & 1.59543 & -1.45323 \\
\hline $\mathrm{H}$ & -2.05156 & 2.98970 & -0.67200 \\
\hline $\mathrm{H}$ & -1.90245 & 3.20296 & 1.90131 \\
\hline $\mathrm{H}$ & -0.46396 & 2.49827 & 2.66578 \\
\hline $\mathrm{H}$ & -1.88467 & 1.48059 & 2.34484 \\
\hline $\mathrm{H}$ & 2.15173 & 4.39849 & -1.80381 \\
\hline $\mathrm{H}$ & 2.96691 & 3.05475 & -2.61049 \\
\hline $\mathrm{H}$ & 1.21809 & 2.98659 & -2.30985 \\
\hline $\mathrm{H}$ & 1.93551 & -3.36298 & -2.02465 \\
\hline $\mathrm{H}$ & 3.26831 & -3.71425 & -0.90575 \\
\hline $\mathrm{H}$ & 1.59263 & -3.77478 & -0.33354 \\
\hline C & -5.31819 & -0.88458 & 0.65374 \\
\hline $\mathrm{H}$ & 2.64004 & -2.08583 & 3.34485 \\
\hline $\mathrm{H}$ & 3.67721 & -0.68790 & 3.05654 \\
\hline $\mathrm{H}$ & 4.41919 & -2.26601 & 3.44082 \\
\hline C & -6.70267 & -1.35595 & 0.27165 \\
\hline 0 & -4.95402 & -0.63902 & 1.78778 \\
\hline $\mathrm{H}$ & -7.17210 & -0.63123 & -0.40190 \\
\hline $\mathrm{H}$ & -6.64286 & -2.30892 & -0.26378 \\
\hline $\mathrm{H}$ & -7.31155 & -1.47158 & 1.16908 \\
\hline
\end{tabular}


Briarellin L - revised

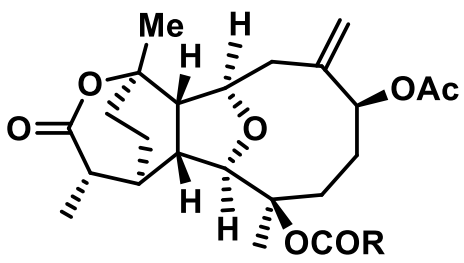

\begin{tabular}{|c|c|c|c|c|c|c|c|c|c|}
\hline & & Rel ene & ergy & $\mathrm{cal} / \mathrm{mol})$ & & $\begin{array}{c}\text { Conf } 1 \\
0.3\end{array}$ & $\begin{array}{c}\text { Conf } 2 \\
0.0\end{array}$ & & \\
\hline iGau & jGau & Jexp & Jcalc & $\operatorname{diff}$ & & 1 & 2 & & \\
\hline 25 & 36 & 11.00 & 11.45 & 0.45 & {[} & 11.68 & 11.30 ] & $\mathrm{H} 1$ & $\mathrm{H} 1 \mathrm{O}$ \\
\hline 25 & 26 & 3.30 & 3.73 & 0.43 & {[} & 3.86 & $3.64]$ & H1 & H2 \\
\hline 39 & 41 & 5.20 & 3.81 & -1.39 & {[} & 4.01 & $3.68 \mathrm{~J}$ & $\mathrm{H} 5 \mathrm{a}$ & H6 \\
\hline 46 & 47 & 13.80 & 14.54 & 0.74 & {[} & -14.55 & $-14.54]$ & $\mathrm{H} 8 \mathrm{a}$ & $\mathrm{H} 8 \mathrm{~b}$ \\
\hline 37 & 47 & 4.80 & 3.81 & -0.99 & {[} & 3.92 & $3.74]$ & H9 & $\mathrm{H} 8 \mathrm{~b}$ \\
\hline 36 & 37 & 8.60 & 7.85 & -0.75 & {[} & 7.53 & $8.05]$ & $\mathrm{H} 10$ & H9 \\
\hline 23 & 24 & 5.20 & 4.68 & -0.52 & {[} & 4.57 & $4.75]$ & H15 & $\mathrm{H} 14$ \\
\hline 20 & 23 & 7.60 & 7.58 & -0.02 & [ & 7.59 & $7.57]$ & $\mathrm{H} 17-\mathrm{Me}$ & $\mathrm{H} 15$ \\
\hline & & For Js: & rms & $=0.77 \mathrm{~Hz}$ & $\mathrm{~N}=8$ & $\{-1,3\}$ & $0.74\}$ & & \\
\hline
\end{tabular}

\begin{tabular}{|c|c|c|c|c|c|c|}
\hline $\mathrm{H}-\mathrm{nom}$ & iGau & $\operatorname{Exp}$ & $\mathrm{Calc}$ & $\operatorname{diff}$ & 1 & 2 \\
\hline $\mathrm{H} 1$ & 25 & 2.53 & 2.69 & 0.16 & 2.82 & 2.61 \\
\hline H2 & 26 & 4.09 & 4.55 & 0.46 & 3.92 & 4.94 \\
\hline $\mathrm{H} 4 \mathrm{a}$ & 30 & 1.58 & 2.05 & 0.47 & 2.58 & 1.72 \\
\hline $\mathrm{H} 4 \mathrm{~b}$ & 31 & 1.58 & 1.74 & 0.16 & 1.53 & 1.88 \\
\hline $\mathrm{H} 5 \mathrm{a}$ & 39 & 1.80 & 1.86 & 0.06 & 2.00 & 1.77 \\
\hline $\mathrm{H} 5 \mathrm{~b}$ & 40 & 2.14 & 2.40 & 0.26 & 2.28 & 2.48 \\
\hline H6 & 41 & 5.15 & 5.61 & 0.46 & 5.49 & 5.69 \\
\hline $\mathrm{H} 8 \mathrm{a}$ & 46 & 3.09 & 2.65 & -0.44 & 2.64 & 2.65 \\
\hline $\mathrm{H} 8 \mathrm{~b}$ & 47 & 2.18 & 2.36 & 0.18 & 2.34 & 2.37 \\
\hline H9 & 37 & 4.13 & 4.37 & 0.24 & 4.39 & 4.35 \\
\hline H1O & 36 & 2.78 & 3.26 & 0.48 & 3.27 & 3.25 \\
\hline $\mathrm{H} 12 \mathrm{a}$ & 33 & 2.02 & 2.26 & 0.24 & 2.28 & 2.24 \\
\hline $\mathrm{H} 12 \mathrm{~b}$ & 32 & 2.02 & 2.21 & 0.19 & 2.22 & 2.20 \\
\hline H13a & 29 & 1.74 & 1.97 & 0.23 & 1.97 & 1.97 \\
\hline $\mathrm{H} 13 \mathrm{~b}$ & 28 & 1.90 & 2.08 & 0.18 & 2.12 & 2.06 \\
\hline H1 4 & 24 & 1.65 & 1.72 & 0.07 & 1.66 & 1.76 \\
\hline H1 5 & 23 & 2.87 & 2.84 & -0.03 & 2.94 & 2.77 \\
\hline $\mathrm{H} 17-\mathrm{Me}$ & 20 & 1.35 & 1.54 & 0.19 & 1.55 & 1.53 \\
\hline $\mathrm{H} 18-\mathrm{Me}$ & 57 & 1.63 & 1.72 & 0.09 & 1.76 & 1.70 \\
\hline H19a & 44 & 5.08 & 5.28 & 0.20 & 5.29 & 5.27 \\
\hline H19b & 45 & 5.32 & 5.74 & 0.42 & 5.91 & 5.63 \\
\hline $\mathrm{H} 20-\mathrm{Me}$ & 48 & 1.29 & 1.42 & 0.13 & 1.43 & 1.42 \\
\hline $\mathrm{H} 22-6 \mathrm{AC}$ & 65 & 1.98 & 2.16 & 0.18 & 2.15 & 2.17 \\
\hline
\end{tabular}

1H chem shifts: $\mathrm{RMSD}=0.28 \mathrm{ppm}(\mathrm{MAE}=0.24) \quad \mathrm{N}=23 \quad\{-0.440 .48\}$

\begin{tabular}{|c|c|c|c|c|c|c|}
\hline C-nom & iGau & Exp & Calc & $\operatorname{diff}$ & 1 & 2 \\
\hline $\mathrm{Cl}-\mathrm{CH}$ & 4 & 44.90 & 45.78 & 0.88 & 45.76 & 45.80 \\
\hline $\mathrm{C} 2-\mathrm{CH}$ & 5 & 90.60 & 93.30 & 2.70 & 95.22 & 92.09 \\
\hline $\mathrm{C} 3-\mathrm{C}$ & 6 & 84.80 & 85.81 & 1.01 & 85.26 & 86.16 \\
\hline $\mathrm{C} 4-\mathrm{CH} 2 * *$ & 10 & 30.10 & 27.65 & -2.45 & 23.64 & 30.18 \\
\hline $\mathrm{C} 5-\mathrm{CH} 2 * *$ & 17 & 28.30 & 26.28 & -2.02 & 25.83 & 26.56 \\
\hline $\mathrm{C} 6-\mathrm{CH}$ & 18 & 74.90 & 75.75 & 0.85 & 76.34 & 75.38 \\
\hline $\mathrm{C} 7-\mathrm{C}$ & 19 & 144.50 & 142.38 & -2.12 & [ 141.79 & 142.75 \\
\hline
\end{tabular}




\begin{tabular}{|c|c|c|c|c|c|c|}
\hline $\mathrm{C} 8-\mathrm{CH} 2$ & 43 & 41.50 & 39.09 & -2.41 & 39.39 & 38.90 \\
\hline $\mathrm{C} 9-\mathrm{CH}$ & 15 & 82.20 & 83.86 & 1.66 & 84.10 & 83.71 \\
\hline $\mathrm{ClO}-\mathrm{CH}$ & 14 & 48.00 & 47.73 & -0.27 & 47.42 & 47.92 \\
\hline $\mathrm{C} 11-\mathrm{C}$ & 13 & 80.90 & 82.01 & 1.11 & 82.00 & 82.02 \\
\hline $\mathrm{C} 12-\mathrm{CH} 2$ & 12 & 30.30 & 30.12 & -0.18 & 29.94 & 30.24 \\
\hline $\mathrm{C} 13-\mathrm{CH} 2$ & 9 & 16.80 & 16.95 & 0.15 & 16.78 & 17.05 \\
\hline $14-\mathrm{CH}$ & 3 & 36.90 & 37.42 & 0.52 & 38.60 & 36.68 \\
\hline $\mathrm{C} 15-\mathrm{CH}$ & 2 & 45.80 & 46.68 & 0.88 & {$[\quad 46.74$} & 46.64 \\
\hline $\mathrm{C} 16-\mathrm{C}$ & 8 & 176.20 & 174.51 & -1.69 & {$[174.30$} & 174.64 \\
\hline $\mathrm{C} 17-\mathrm{CH} 3$ & 1 & 17.40 & 17.99 & 0.59 & [ 18.06 & 17.94 \\
\hline $\mathrm{C} 18-\mathrm{CH} 3$ & 27 & 20.50 & 20.99 & 0.49 & 22.31 & 20.16 \\
\hline $\mathrm{C} 19-\mathrm{CH} 2$ & 42 & 118.60 & 118.71 & 0.11 & [ 119.40 & 118.28 \\
\hline $\mathrm{C} 20-\mathrm{CH} 3$ & 34 & 28.90 & 27.92 & -0.98 & 27.93 & 27.91 \\
\hline $\mathrm{C}-\mathrm{Acyl}-\mathrm{C}$ & 60 & 170.70 & 170.11 & -0.59 & 170.11 & 170.11 \\
\hline $\mathrm{C}-\mathrm{Acyl}-\mathrm{CH} 3$ & 61 & 21.30 & 20.45 & -0.85 & 20.44 & 20.46 \\
\hline $\mathrm{C}-\mathrm{Acyl}-\mathrm{C}$ & 50 & 172.40 & 170.71 & -1.69 & 169.98 & 171.17 \\
\hline
\end{tabular}

13C chem shifts: $R M S D=1.38 \mathrm{ppm}(\mathrm{MAE}=1.14) \mathrm{N}=23 \quad\left\{\begin{array}{l}-2.452 .70 \\ 2\end{array}\right.$

Fractions: 0.3870 .613

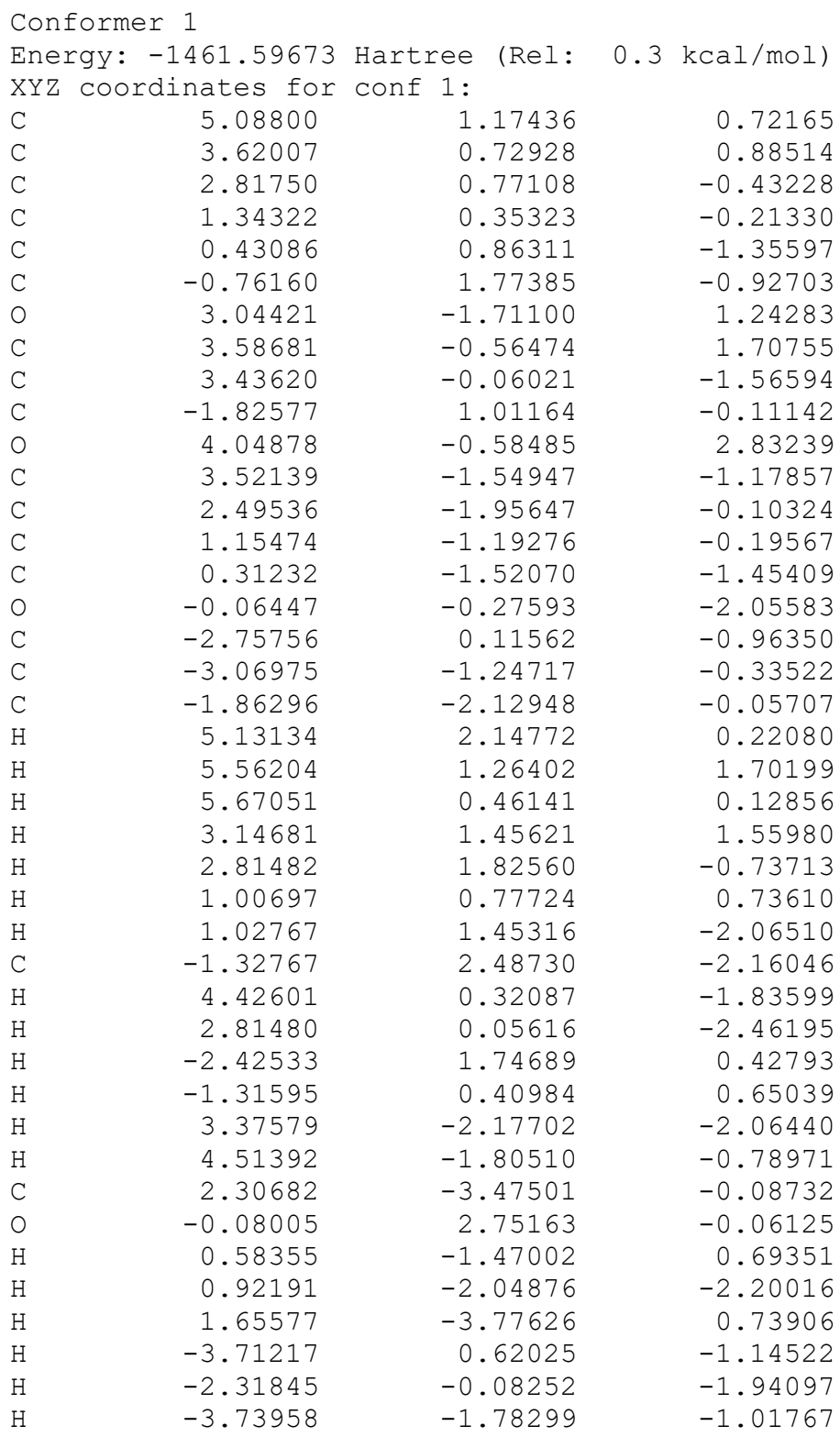




$\begin{array}{lrrr}\mathrm{C} & -1.67260 & -2.70390 & 1.13729 \\ \mathrm{C} & -0.93201 & -2.42829 & -1.22413 \\ \mathrm{H} & -0.85044 & -3.39516 & 1.30356 \\ \mathrm{H} & -2.33270 & -2.51625 & 1.97604 \\ \mathrm{H} & -1.49270 & -2.43433 & -2.16764 \\ \mathrm{H} & -0.54767 & -3.44604 & -1.09329 \\ \mathrm{H} & 1.87179 & -3.82764 & -1.02697 \\ \mathrm{H} & 3.27575 & -3.96744 & 0.04384 \\ \mathrm{C} & -0.71117 & 3.82230 & 0.46686 \\ \mathrm{C} & 0.25245 & 4.63563 & 1.30296 \\ \mathrm{O} & -1.88392 & 4.09555 & 0.30546 \\ \mathrm{H} & 1.12809 & 4.91717 & 0.70972 \\ \mathrm{H} & -0.25051 & 5.53023 & 1.67189 \\ \mathrm{H} & 0.60759 & 4.03909 & 2.15021 \\ \mathrm{H} & -2.22201 & 3.06215 & -1.91603 \\ \mathrm{H} & -0.57372 & 3.16864 & -2.56992 \\ \mathrm{H} & -1.57201 & 1.75641 & -2.93600 \\ \mathrm{O} & -3.79065 & -1.04711 & 0.90879 \\ \mathrm{C} & -5.14061 & -0.99267 & 0.85017 \\ \mathrm{C} & -5.72616 & -0.76446 & 2.22302 \\ \mathrm{O} & -5.78187 & -1.11250 & -0.17469 \\ \mathrm{H} & -5.34792 & 0.17447 & 2.64035 \\ \mathrm{H} & -5.42354 & -1.56962 & 2.90050 \\ \mathrm{H} & -6.81392 & -0.72701 & 2.15568\end{array}$

Conformer 2

Energy: -1461.59716 Hartree (Rel: $0.0 \mathrm{kcal} / \mathrm{mol}$ ) XYZ coordinates for conf 2 :

$\begin{array}{lrrr}\mathrm{C} & 5.14161 & -0.05080 & 0.72950 \\ \mathrm{C} & 3.61112 & -0.10465 & 0.91522 \\ \mathrm{C} & 2.82619 & 0.25969 & -0.36280 \\ \mathrm{C} & 1.29618 & 0.20451 & -0.11949 \\ \mathrm{C} & 0.51559 & 1.04999 & -1.15660 \\ \mathrm{C} & -0.46192 & 2.12545 & -0.57604 \\ \mathrm{O} & 2.42445 & -2.33388 & 1.09599 \\ \mathrm{C} & 3.24709 & -1.40794 & 1.63569 \\ \mathrm{C} & 3.19607 & -0.59449 & -1.58346 \\ \mathrm{C} & -1.70403 & 1.50297 & 0.09550 \\ \mathrm{O} & 3.69126 & -1.63828 & 2.74432 \\ \mathrm{C} & 2.92223 & -2.08643 & -1.31692 \\ \mathrm{C} & 1.83119 & -2.31887 & -0.25337 \\ \mathrm{C} & 0.72395 & -1.23990 & -0.25483 \\ \mathrm{C} & -0.14292 & -1.21992 & -1.53820 \\ \mathrm{O} & -0.23329 & 0.14207 & -1.97184 \\ \mathrm{C} & -2.78783 & 0.97579 & -0.87268 \\ \mathrm{C} & -3.42257 & -0.35090 & -0.44365 \\ \mathrm{C} & -2.44150 & -1.49686 & -0.24396 \\ \mathrm{H} & 5.43346 & 0.91513 & 0.30314 \\ \mathrm{H} & 5.63974 & -0.17200 & 1.69452 \\ \mathrm{H} & 5.50255 & -0.83964 & 0.06097 \\ \mathrm{H} & 3.36131 & 0.66107 & 1.66284 \\ \mathrm{H} & 3.08610 & 1.30193 & -0.57921 \\ \mathrm{H} & 1.09368 & 0.58316 & 0.88680 \\ \mathrm{H} & 1.21606 & 1.57330 & -1.81356 \\ \mathrm{C} & -0.84680 & 3.15434 & -1.64395 \\ \mathrm{H} & 4.24345 & -0.44296 & -1.86310 \\ \mathrm{H} & 2.60419 & -0.25116 & -2.44057 \\ \mathrm{H} & -2.13270 & 2.27395 & 0.74343 \\ \mathrm{H} & -1.37368 & 0.70199 & 0.76716 \\ \mathrm{H} & 2.62808 & -2.58803 & -2.24525 \\ \mathrm{H} & 3.82511 & -2.60288 & -0.97058 \\ \mathrm{C} & 1.27291 & -3.73951 & -0.36160 \\ \mathrm{O} & 0.17068 & 2.83169 & 0.55771 \\ & & & \end{array}$




$\begin{array}{rrrr}\mathrm{H} & 0.08243 & -1.45145 & 0.60422 \\ \mathrm{H} & 0.35980 & -1.76953 & -2.34641 \\ \mathrm{H} & 0.56883 & -3.94442 & 0.45072 \\ \mathrm{H} & -3.59520 & 1.70838 & -0.98015 \\ \mathrm{H} & -2.36987 & 0.81693 & -1.86609 \\ \mathrm{H} & -4.14695 & -0.65248 & -1.20773 \\ \mathrm{C} & -2.41300 & -2.20761 & 0.88980 \\ \mathrm{C} & -1.55575 & -1.86368 & -1.42525 \\ \mathrm{H} & -1.76113 & -3.07028 & 0.99925 \\ \mathrm{H} & -3.04024 & -1.96108 & 1.73907 \\ \mathrm{H} & -2.07326 & -1.64761 & -2.36880 \\ \mathrm{H} & -1.39986 & -2.94776 & -1.40602 \\ \mathrm{H} & 0.76369 & -3.88978 & -1.31796 \\ \mathrm{H} & 2.09097 & -4.46402 & -0.29529 \\ \mathrm{C} & 1.30973 & 3.54008 & 0.43502 \\ \mathrm{C} & 1.62598 & 4.25542 & 1.72940 \\ \mathrm{O} & 1.99481 & 3.59724 & -0.56957 \\ \mathrm{H} & 1.65668 & 3.54342 & 2.56020 \\ \mathrm{H} & 2.58545 & 4.76610 & 1.64036 \\ \mathrm{H} & 0.83982 & 4.98438 & 1.95307 \\ \mathrm{H} & -1.55882 & 3.87968 & -1.23538 \\ \mathrm{H} & 0.03525 & 3.68725 & -2.00364 \\ \mathrm{H} & -1.31406 & 2.65598 & -2.49761 \\ \mathrm{O} & -4.16387 & -0.10815 & 0.77968 \\ \mathrm{C} & -5.47045 & -0.46291 & 0.81978 \\ \mathrm{C} & -6.06656 & -0.13935 & 2.16875 \\ \mathrm{O} & -6.06866 & -0.97487 & -0.10437 \\ \mathrm{H} & -5.84987 & 0.89694 & 2.44453 \\ \mathrm{H} & -5.61899 & -0.78315 & 2.93413 \\ \mathrm{H} & -7.14418 & -0.30452 & 2.14239 \\ & & & \end{array}$


Briarellins M - revised
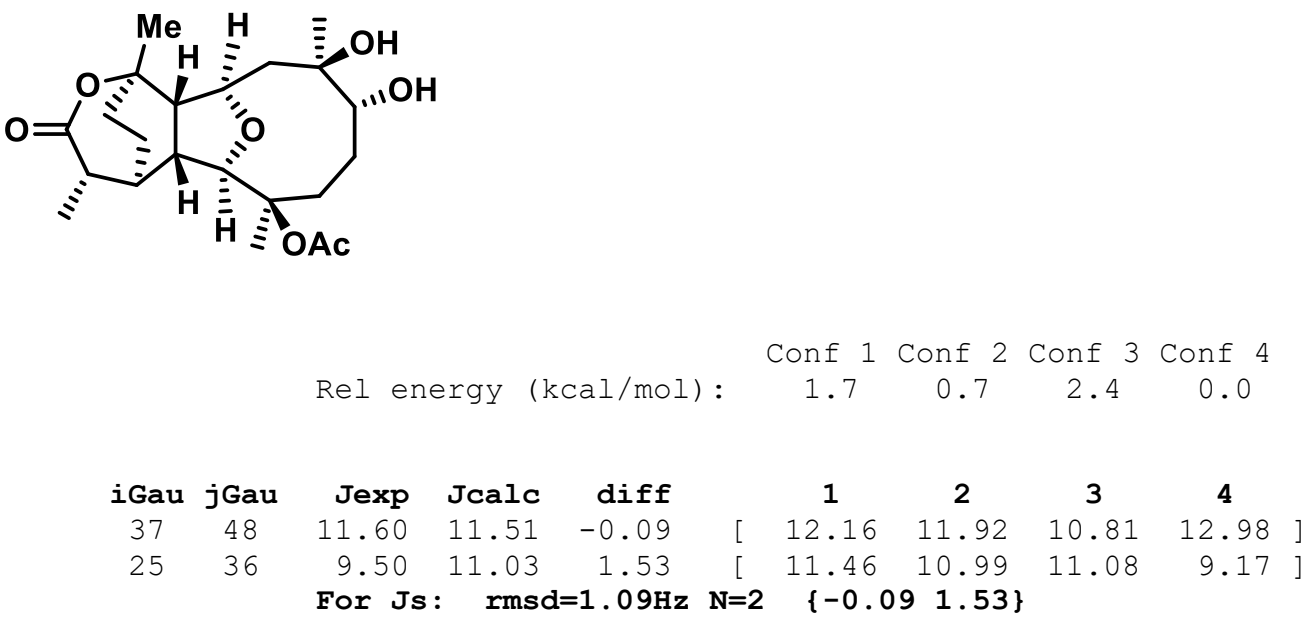

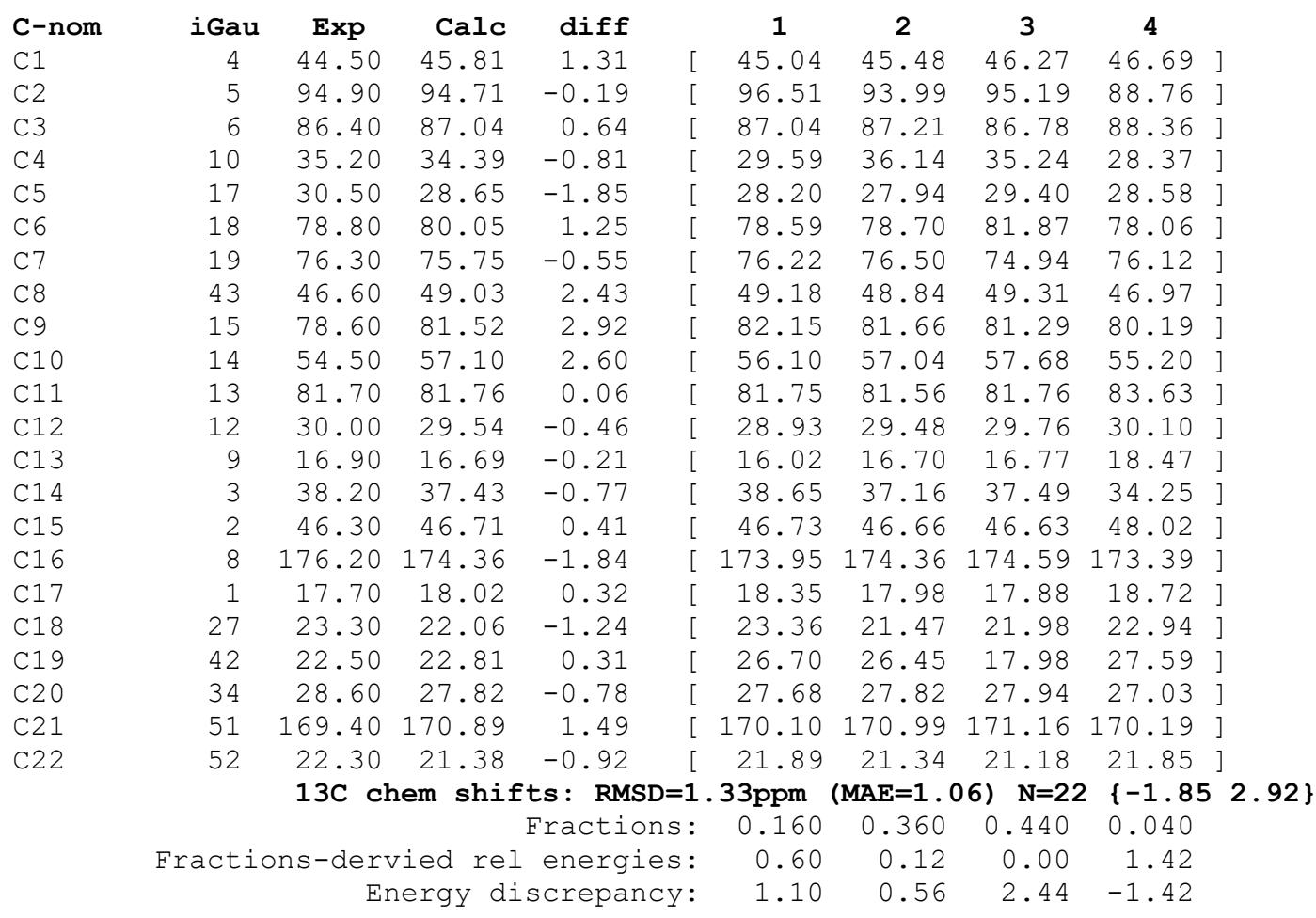

(this is the best fit)

Conformer 1

Energy: -1385.36954 Hartree (Rel: $1.7 \mathrm{kcal} / \mathrm{mol}$ )

XYZ coordinates for conf 1 :

$\begin{array}{rrrr}\text { C } & 4.82633 & 0.96950 & 0.70933 \\ \text { C } & 3.52668 & 0.63716 & -0.05303 \\ \text { C } & 2.29389 & 0.52994 & 0.86845 \\ \text { C } & 0.98971 & 0.31566 & 0.05996 \\ \text { C } & -0.25603 & 0.65480 & 0.91965 \\ \text { C } & -1.23486 & 1.74614 & 0.37098 \\ \text { O } & 3.10529 & -1.66798 & -1.00916 \\ \text { C } & 3.81039 & -0.51652 & -1.02049\end{array}$




\begin{tabular}{|c|c|c|}
\hline 2.41816 & -0.53768 & 1.96673 \\
\hline-2.06078 & 1.29533 & -0.85645 \\
\hline 4.69158 & -0.41415 & -1.85220 \\
\hline 2.52764 & -1.95971 & 1.37554 \\
\hline 2.03200 & -2.05869 & -0.07670 \\
\hline 0.80868 & -1.16806 & -0.39170 \\
\hline-0.53351 & -1.62955 & 0.23924 \\
\hline-0.93221 & -0.58272 & 1.15006 \\
\hline-3.44176 & 0.60364 & -0.67033 \\
\hline-3.70933 & -0.71964 & 0.09541 \\
\hline-3.06657 & -2.02289 & -0.48474 \\
\hline 5.63094 & 1.17911 & 0.00088 \\
\hline 4.67636 & 1.85193 & 1.34076 \\
\hline 5.14887 & 0.14288 & 1.35107 \\
\hline 3.33666 & 1.48310 & -0.72877 \\
\hline 2.21263 & 1.51163 & 1.35267 \\
\hline 1.01778 & 0.96685 & -0.81621 \\
\hline 0.06736 & 1.01143 & 1.90432 \\
\hline-2.08759 & 2.31059 & 1.51136 \\
\hline 3.27504 & -0.32604 & 2.61350 \\
\hline 1.53406 & -0.47705 & 2.61140 \\
\hline-2.29732 & 2.20960 & -1.40600 \\
\hline-1.41121 & 0.71667 & -1.51906 \\
\hline 1.95427 & -2.66396 & 1.98842 \\
\hline 3.56219 & -2.32091 & 1.38830 \\
\hline 1.80237 & -3.51836 & -0.47869 \\
\hline-0.26324 & 2.77691 & -0.05417 \\
\hline 0.70529 & -1.20266 & -1.48028 \\
\hline-0.41025 & -2.51134 & 0.87544 \\
\hline 1.01508 & -3.97864 & 0.12474 \\
\hline-4.11795 & 1.33811 & -0.21624 \\
\hline-3.82017 & 0.43997 & -1.68719 \\
\hline-3.56787 & -0.61206 & 1.50863 \\
\hline-3.35533 & -3.21453 & 0.43882 \\
\hline-1.57253 & -1.93637 & -0.85217 \\
\hline-3.02870 & -4.14079 & -0.04608 \\
\hline-4.43032 & -3.29187 & 0.64436 \\
\hline-2.84968 & -3.12205 & 1.40385 \\
\hline-1.29139 & -2.90473 & -1.28694 \\
\hline-1.48135 & -1.21562 & -1.66761 \\
\hline 1.52332 & -3.58792 & -1.53477 \\
\hline 2.72316 & -4.09089 & -0.32946 \\
\hline-0.62514 & 3.99505 & -0.51177 \\
\hline 0.60477 & 4.81491 & -0.83491 \\
\hline-1.76769 & 4.38236 & -0.65575 \\
\hline 0.30254 & 5.80793 & -1.16949 \\
\hline 1.24794 & 4.89845 & 0.04689 \\
\hline 1.18710 & 4.32325 & -1.62147 \\
\hline-2.75130 & 3.09638 & 1.14751 \\
\hline-1.43912 & 2.73264 & 2.28724 \\
\hline-2.69921 & 1.52033 & 1.95140 \\
\hline-4.78844 & -0.88282 & -0.04009 \\
\hline-3.67405 & -2.25949 & -1.77306 \\
\hline-4.61730 & -2.43695 & -1.6210 \\
\hline-2.60456 & -0.60313 & 1.676 \\
\hline
\end{tabular}

Conformer 2

Energy: -1385.37116 Hartree (Rel: $0.7 \mathrm{kcal} / \mathrm{mol}$ ) XYZ coordinates for conf 2 :

$\begin{array}{rrrr}\text { C } & 4.80961 & -0.01960 & 0.79472 \\ \text { C } & 3.50415 & -0.02707 & -0.02770 \\ \text { C } & 2.23946 & 0.17397 & 0.83444 \\ \text { C } & 0.95879 & 0.21349 & -0.03949\end{array}$




\begin{tabular}{|c|c|c|c|}
\hline $\mathrm{C}$ & -0.21401 & 0.90045 & 0.70667 \\
\hline C & -0.97662 & 2.04991 & -0.03752 \\
\hline 0 & 2.59957 & -2.18659 & -0.98919 \\
\hline $\mathrm{C}$ & 3.53995 & -1.21673 & -0.99276 \\
\hline $\mathrm{C}$ & 2.07925 & -0.85803 & 1.96066 \\
\hline $\mathrm{C}$ & -1.90328 & 1.55789 & -1.17692 \\
\hline 0 & 4.43194 & -1.31201 & -1.81378 \\
\hline C & 1.96291 & -2.28960 & 1.39969 \\
\hline $\mathrm{C}$ & 1.46719 & -2.32883 & -0.05733 \\
\hline $\mathrm{C}$ & 0.44940 & -1.21858 & -0.39987 \\
\hline C & -0.92858 & -1.36350 & 0.30335 \\
\hline 0 & -1.12702 & -0.14848 & 1.05533 \\
\hline $\mathrm{C}$ & -3.38757 & 1.17989 & -0.91234 \\
\hline $\mathrm{C}$ & -3.88820 & 0.07561 & 0.05582 \\
\hline C & -3.51714 & -1.40309 & -0.30139 \\
\hline $\mathrm{H}$ & 5.67234 & -0.01047 & 0.12433 \\
\hline $\mathrm{H}$ & 4.84813 & 0.87289 & 1.42847 \\
\hline $\mathrm{H}$ & 4.89399 & -0.89976 & 1.44087 \\
\hline $\mathrm{H}$ & 3.55894 & 0.83681 & -0.70497 \\
\hline $\mathrm{H}$ & 2.35283 & 1.16476 & 1.28774 \\
\hline $\mathrm{H}$ & 1.18146 & 0.76115 & -0.95935 \\
\hline $\mathrm{H}$ & 0.14182 & 1.31633 & 1.65150 \\
\hline C & -1.71686 & 2.96386 & 0.94449 \\
\hline $\mathrm{H}$ & 2.91179 & -0.79267 & 2.66802 \\
\hline $\mathrm{H}$ & 1.17841 & -0.60921 & 2.53356 \\
\hline $\mathrm{H}$ & -1.95761 & 2.40412 & -1.87136 \\
\hline $\mathrm{H}$ & -1.38447 & 0.77422 & -1.73564 \\
\hline $\mathrm{H}$ & 1.28460 & -2.88379 & 2.02164 \\
\hline $\mathrm{H}$ & 2.92758 & -2.80964 & 1.42536 \\
\hline $\mathrm{C}$ & 0.94474 & -3.72101 & -0.42068 \\
\hline 0 & -0.00109 & 2.87272 & -0.79219 \\
\hline $\mathrm{H}$ & 0.29470 & -1.28364 & -1.48117 \\
\hline $\mathrm{H}$ & -0.91728 & -2.15655 & 1.05889 \\
\hline $\mathrm{H}$ & 0.06397 & -3.98100 & 0.17352 \\
\hline $\mathrm{H}$ & -3.91049 & 2.09193 & -0.60031 \\
\hline $\mathrm{H}$ & -3.79856 & 0.92432 & -1.89725 \\
\hline 0 & -3.69604 & 0.38620 & 1.43197 \\
\hline C & -3.96756 & -2.35239 & 0.81706 \\
\hline C & -2.05135 & -1.65294 & -0.70684 \\
\hline $\mathrm{H}$ & -3.83391 & -3.38973 & 0.49166 \\
\hline $\mathrm{H}$ & -5.02776 & -2.19687 & 1.05145 \\
\hline $\mathrm{H}$ & -3.40771 & -2.19699 & 1.74331 \\
\hline $\mathrm{H}$ & -1.96859 & -2.71324 & -0.97833 \\
\hline $\mathrm{H}$ & -1.87594 & -1.10256 & -1.63349 \\
\hline $\mathrm{H}$ & 0.68010 & -3.76896 & -1.48156 \\
\hline $\mathrm{H}$ & 1.71940 & -4.46945 & -0.22585 \\
\hline C & 1.01032 & 3.52947 & -0.19198 \\
\hline C & 1.73626 & 4.40413 & -1.18918 \\
\hline 0 & 1.30818 & 3.43004 & 0.98402 \\
\hline $\mathrm{H}$ & 2.57547 & 4.89882 & -0.69900 \\
\hline $\mathrm{H}$ & 2.09772 & 3.80206 & -2.02910 \\
\hline $\mathrm{H}$ & 1.05072 & 5.15446 & -1.59664 \\
\hline $\mathrm{H}$ & -2.18332 & 3.79248 & 0.40016 \\
\hline $\mathrm{H}$ & -1.03140 & 3.37533 & 1.68778 \\
\hline $\mathrm{H}$ & -2.50731 & 2.40788 & 1.45336 \\
\hline $\mathrm{H}$ & -4.98138 & 0.10138 & -0.06154 \\
\hline O & -4.22032 & -1.73288 & -1.51903 \\
\hline $\mathrm{H}$ & -5.17120 & -1.70716 & -1.32004 \\
\hline $\mathrm{H}$ & -2.74402 & 0.23700 & 1.59960 \\
\hline
\end{tabular}

Conformer 3

Energy: -1385.36836 Hartree (Rel: $2.4 \mathrm{kcal} / \mathrm{mol}$ ) XYZ coordinates for conf 3 : 


\begin{tabular}{|c|c|c|}
\hline 4.88537 & -0.18304 & 0.80236 \\
\hline 3.56854 & -0.09752 & 0.00323 \\
\hline 2.32349 & 0.09393 & 0.89504 \\
\hline 1.03456 & 0.22285 & 0.04243 \\
\hline-0.10578 & 0.91491 & 0.83403 \\
\hline-0.82514 & 2.15273 & 0.18053 \\
\hline 2.57095 & -2.15744 & -1.07838 \\
\hline 3.54815 & -1.22602 & -1.03407 \\
\hline 2.14505 & -0.99705 & 1.96076 \\
\hline-1.98422 & 1.72653 & -0.74633 \\
\hline 4.42544 & -1.30167 & -1.87322 \\
\hline 1.98780 & -2.38804 & 1.31566 \\
\hline 1.45586 & -2.32496 & -0.12830 \\
\hline 0.46488 & -1.16716 & -0.37542 \\
\hline-0.89094 & -1.30356 & 0.37125 \\
\hline-1.08727 & -0.08722 & 1.10883 \\
\hline-3.31001 & 1.31019 & -0.05217 \\
\hline-3.98209 & 0.06446 & -0.64967 \\
\hline-3.49903 & -1.34099 & -0.17319 \\
\hline 5.73811 & -0.16459 & 0.11945 \\
\hline 4.96480 & 0.66961 & 1.48531 \\
\hline 4.94820 & -1.10138 & 1.39567 \\
\hline 3.64258 & 0.80450 & -0.62007 \\
\hline 2.47374 & 1.05431 & 1.39905 \\
\hline 1.26753 & 0.79736 & -0.85750 \\
\hline 0.27950 & 1.24902 & 1.80216 \\
\hline-1.29692 & 3.13921 & 1.25595 \\
\hline 2.98454 & -0.99558 & 2.66316 \\
\hline 1.25487 & -0.75957 & 2.55519 \\
\hline-2.18411 & 2.58098 & -1.40125 \\
\hline-1.62138 & 0.93257 & -1.40580 \\
\hline 1.31394 & -3.00849 & 1.91632 \\
\hline 2.94301 & -2.92520 & 1.28483 \\
\hline 0.88559 & -3.67827 & -0.55812 \\
\hline 0.06262 & 2.87265 & -0.75277 \\
\hline 0.27180 & -1.16586 & -1.45282 \\
\hline-0.84503 & -2.09456 & 1.12985 \\
\hline 0.01523 & -3.94865 & 0.04663 \\
\hline-3.16433 & 1.15646 & 1.01813 \\
\hline-4.03202 & 2.12761 & -0.15991 \\
\hline-5.41190 & 0.12568 & -0.47214 \\
\hline-3.75131 & -1.59701 & 1.32168 \\
\hline-2.04577 & -1.63960 & -0.59807 \\
\hline-3.17543 & -0.92830 & 1.96582 \\
\hline-4.81688 & -1.48489 & 1.55343 \\
\hline-3.47863 & -2.63034 & 1.56120 \\
\hline-1.99771 & -2.72055 & -0.77714 \\
\hline-1.87084 & -1.17518 & -1.57581 \\
\hline 0.58899 & -3.65677 & -1.61143 \\
\hline 1.64410 & -4.45733 & -0.43070 \\
\hline 1.21903 & 3.43193 & -0.34754 \\
\hline 1.84092 & 4.23550 & -1.46665 \\
\hline 1.69966 & 3.30505 & 0.76350 \\
\hline 1.95328 & 3.61785 & -2.36325 \\
\hline 2.81366 & 4.61290 & -1.14938 \\
\hline 1.18779 & 5.07502 & -1.72799 \\
\hline-1.84895 & 3.96787 & 0.79924 \\
\hline-0.44805 & 3.54057 & 1.81179 \\
\hline-1.95866 & 2.63692 & 1.96710 \\
\hline-3.85919 & 0.07112 & -1.73908 \\
\hline-4.28619 & -2.28038 & -0.93048 \\
\hline-5.18982 & -1.91379 & -0.90642 \\
\hline-5.58927 & 0.32884 & 0.46115 \\
\hline
\end{tabular}




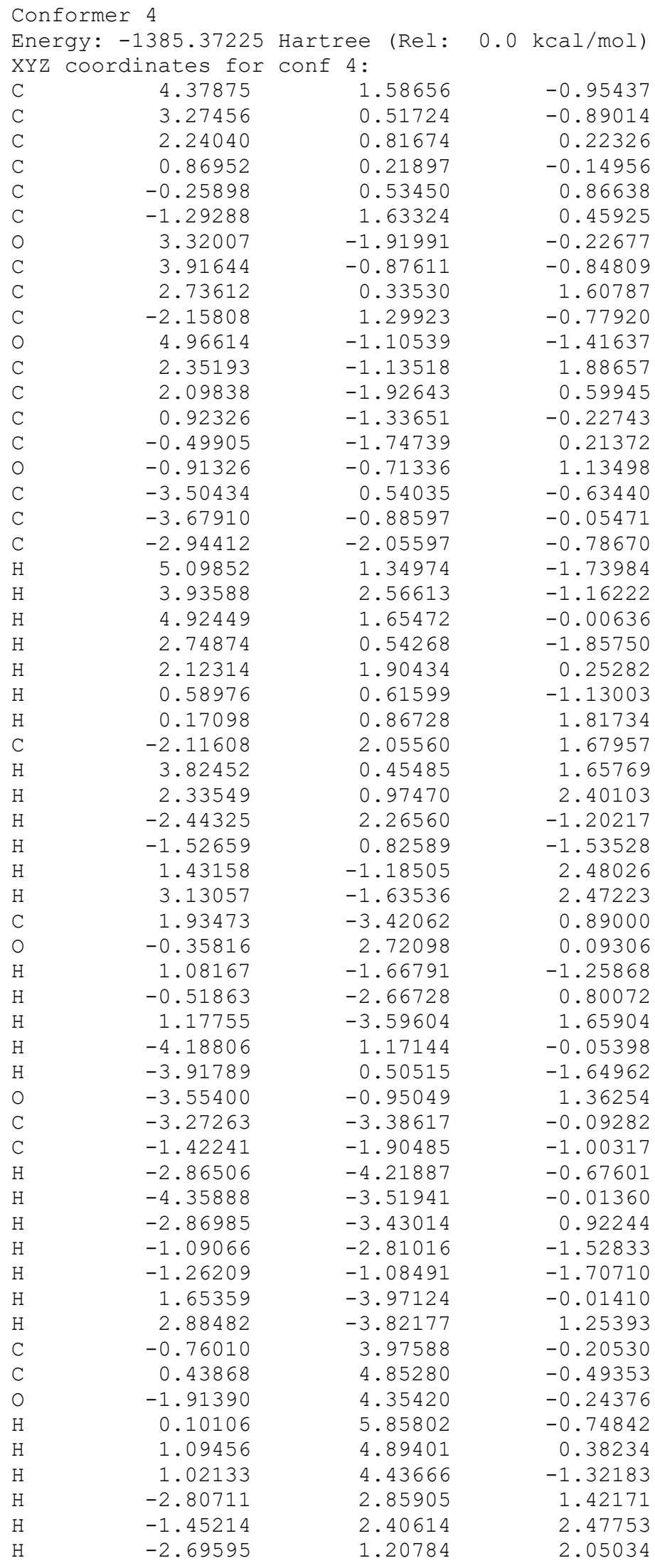




$\begin{array}{llll}\mathrm{H} & -4.74328 & -1.10601 & -0.22604 \\ \mathrm{O} & -3.43865 & -2.08969 & -2.14231 \\ \mathrm{H} & -4.38595 & -2.30271 & -2.10131 \\ \mathrm{H} & -2.59864 & -0.86118 & 1.55193\end{array}$


Asbestinin-21 - original

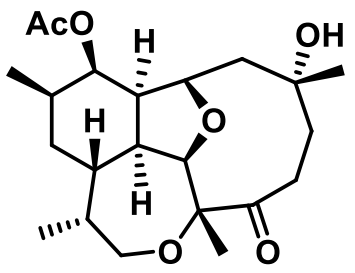

Rel energy (kcal/mol): $\begin{array}{cccc}\text { Conf } 1 & \text { Conf } 2 & \text { Conf } 3 & \text { Conf } 4 \\ 0.0 & 0.4 & 0.5 & 1.4\end{array}$

\begin{tabular}{|c|c|c|c|c|c|c|c|c|c|}
\hline C-nom & iGau & $\operatorname{Exp}$ & Calc & $\operatorname{diff}$ & 1 & 2 & 3 & 4 & \\
\hline $\mathrm{Cl}-\mathrm{CH}$ & 4 & 38.03 & 37.80 & -0.23 & 37.85 & 37.33 & 37.29 & 42.11 & ] \\
\hline $\mathrm{C} 2-\mathrm{CH}$ & 5 & 93.64 & 92.45 & -1.19 & 93.09 & 91.67 & 91.96 & 92.05 & ] \\
\hline C3-C & 6 & 77.21 & 85.71 & 8.50 & 85.65 & 85.64 & 85.83 & 86.24 & ] \\
\hline $\mathrm{C} 4-\mathrm{C}$ & 10 & 210.78 & 215.03 & 4.25 & [ 214.08 & 216.51 & 216.25 & 211.54 & ] \\
\hline $\mathrm{C} 5-\mathrm{CH} 2$ & 17 & 36.19 & 37.70 & 1.51 & {$[\quad 36.72$} & 39.32 & 39.11 & 33.07 & ] \\
\hline $\mathrm{C} 6-\mathrm{CH} 2$ & 18 & 34.03 & 37.58 & 3.55 & 38.62 & 35.38 & 37.12 & 40.19 & ] \\
\hline $\mathrm{C} 7-\mathrm{C}$ & 19 & 76.29 & 73.09 & -3.20 & 73.13 & 72.86 & 73.14 & 73.54 & ] \\
\hline $\mathrm{C} 8-\mathrm{CH} 2$ & 42 & 49.33 & 48.30 & -1.03 & 50.60 & 46.14 & 46.25 & 45.12 & ] \\
\hline $\mathrm{C} 9-\mathrm{CH}$ & 15 & 78.23 & 79.99 & 1.76 & 79.81 & 80.03 & 79.85 & 82.31 & ] \\
\hline $\mathrm{ClO}-\mathrm{CH}$ & 14 & 47.73 & 49.16 & 1.43 & 49.23 & 49.47 & 49.55 & 44.97 & ] \\
\hline $\mathrm{C} 11-\mathrm{CH}$ & 13 & 72.79 & 73.32 & 0.53 & 73.23 & 73.38 & 73.32 & 74.02 & ] \\
\hline $\mathrm{C} 12-\mathrm{CH}$ & 12 & 31.51 & 32.56 & 1.05 & 32.55 & 32.55 & 32.56 & 32.63 & ] \\
\hline $\mathrm{C} 13-\mathrm{CH} 2$ & 9 & 31.51 & 30.66 & -0.85 & 30.69 & 30.55 & 30.53 & 31.43 & ] \\
\hline $\mathrm{Cl} 4-\mathrm{CH}$ & 3 & 38.03 & 38.81 & 0.78 & 38.68 & 39.08 & 39.11 & 37.29 & ] \\
\hline $\mathrm{C} 15-\mathrm{CH}$ & 2 & 36.52 & 37.50 & 0.98 & 37.14 & 37.96 & 37.83 & 37.33 & ] \\
\hline $\mathrm{C} 16-\mathrm{CH} 2$ & 8 & 68.17 & 68.86 & 0.69 & 68.80 & 68.61 & 68.71 & 71.43 & ] \\
\hline $\mathrm{C} 17-\mathrm{CH} 3$ & 1 & 10.91 & 11.07 & 0.16 & 11.14 & 10.88 & 10.90 & 12.16 & ] \\
\hline $\mathrm{C} 18-\mathrm{CH} 3$ & 27 & 22.50 & 20.42 & -2.08 & 19.52 & 21.41 & 21.42 & 19.98 & ] \\
\hline $\mathrm{C} 19-\mathrm{CH} 3$ & 41 & 27.66 & 26.90 & -0.76 & 24.95 & 29.03 & 26.75 & 36.80 & ] \\
\hline $\mathrm{C} 2 \mathrm{O}-\mathrm{CH} 3$ & 32 & 17.25 & 17.08 & -0.17 & 16.94 & 17.30 & 17.25 & 16.66 & ] \\
\hline $\mathrm{C} 21-\mathrm{C}$ & 49 & 170.84 & 171.55 & 0.71 & [ 171.49 & 171.66 & 171.57 & 171.52 & ] \\
\hline \multirow[t]{3}{*}{$\mathrm{C} 22-\mathrm{CH} 3$} & 50 & 21.34 & 20.84 & -0.50 & [ 20.83 & 20.87 & 20.87 & 20.62 & ] \\
\hline & & $13 \mathrm{C} \mathrm{c}$ & em shif & S: RMS & $2.46 \mathrm{ppm}$ & $(\mathrm{MAE}=1.6$ & 53) $\mathrm{N}=22$ & $\{-3.20$ & $8.50\}$ \\
\hline & & & & Fractio & $: \quad 0.490$ & 0.244 & 0.219 & 0.047 & \\
\hline
\end{tabular}

Conformer 1

Energy: -1310.13728 Hartree (Rel: $0.0 \mathrm{kcal} / \mathrm{mol}$ ) XYZ coordinates for conf 1 :

$\begin{array}{lrrr}\text { C } & 1.57593 & 3.63989 & -1.43784 \\ \text { C } & 1.96366 & 2.85245 & -0.17633 \\ \text { C } & 2.05103 & 1.31734 & -0.36520 \\ \text { C } & 0.65151 & 0.69297 & -0.51594 \\ \text { C } & -0.06775 & 0.56203 & 0.83328 \\ \text { C } & -0.98083 & 1.78955 & 1.14511 \\ \text { O } & -0.36306 & 3.00447 & 0.71927 \\ \text { C } & 1.02678 & 3.21861 & 0.99792 \\ \text { C } & 2.94881 & 0.83373 & -1.51464 \\ \text { C } & -2.25015 & 1.62629 & 0.27038 \\ \text { H } & 1.11523 & 4.29289 & 1.19739 \\ \text { C } & 3.14046 & -0.71571 & -1.50022 \\ \text { C } & 1.96314 & -1.46781 & -0.82445 \\ \text { C } & 0.64064 & -0.74573 & -1.08763 \\ \text { C } & -0.63023 & -1.35471 & -0.45087 \\ \text { O } & -0.81785 & -0.65673 & 0.79944 \\ \text { C } & -3.36659 & 0.69729 & 0.74676 \\ \text { C } & -3.98910 & -0.14860 & -0.37668 \\ \text { C } & -3.27494 & -1.45169 & -0.81110 \\ \text { H } & 2.26728 & 3.44487 & -2.26412\end{array}$




\begin{tabular}{|c|c|c|c|}
\hline $\mathrm{H}$ & 1.60234 & 4.71685 & -1.23237 \\
\hline $\mathrm{H}$ & 0.56168 & 3.39788 & -1.76896 \\
\hline $\mathrm{H}$ & 2.96365 & 3.19269 & 0.13523 \\
\hline $\mathrm{H}$ & 2.48890 & 0.92332 & 0.56195 \\
\hline $\mathrm{H}$ & 0.04782 & 1.32142 & -1.17645 \\
\hline $\mathrm{H}$ & 0.66220 & 0.46583 & 1.64775 \\
\hline C & -1.31299 & 1.87607 & 2.64361 \\
\hline $\mathrm{H}$ & 2.50854 & 1.13685 & -2.47266 \\
\hline $\mathrm{H}$ & 3.93061 & 1.32126 & -1.46046 \\
\hline $\mathrm{H}$ & -5.00117 & -0.43906 & -0.07293 \\
\hline $\mathrm{H}$ & 3.11914 & -1.05729 & -2.54375 \\
\hline $\mathrm{C}$ & 4.49638 & -1.13320 & -0.91346 \\
\hline $\mathrm{H}$ & 1.92620 & -2.49639 & -1.18876 \\
\hline 0 & 2.18835 & -1.53961 & 0.61676 \\
\hline $\mathrm{H}$ & 0.52747 & -0.71670 & -2.17816 \\
\hline $\mathrm{H}$ & -0.48587 & -2.40720 & -0.18788 \\
\hline $\mathrm{H}$ & 4.60470 & -2.22432 & -0.89219 \\
\hline $\mathrm{H}$ & -3.04128 & 0.07671 & 1.58028 \\
\hline $\mathrm{H}$ & -4.14888 & 1.36562 & 1.13765 \\
\hline $\mathrm{H}$ & -4.09681 & 0.48072 & -1.26643 \\
\hline C & -3.30491 & -2.52568 & 0.28453 \\
\hline C & -1.84642 & -1.22416 & -1.38933 \\
\hline $\mathrm{H}$ & -2.87332 & -3.46562 & -0.08499 \\
\hline $\mathrm{H}$ & -2.73618 & -2.22660 & 1.16888 \\
\hline $\mathrm{H}$ & -1.83077 & -0.25218 & -1.89620 \\
\hline $\mathrm{H}$ & -1.69460 & -1.97611 & -2.17666 \\
\hline $\mathrm{H}$ & 5.31186 & -0.72604 & -1.52125 \\
\hline $\mathrm{H}$ & 4.62017 & -0.76416 & 0.11010 \\
\hline C & 2.00502 & -2.72409 & 1.24260 \\
\hline C & 2.22147 & -2.58122 & 2.72987 \\
\hline 0 & 1.68992 & -3.75487 & 0.68084 \\
\hline $\mathrm{H}$ & 3.12688 & -2.00497 & 2.93943 \\
\hline $\mathrm{H}$ & -2.04316 & 2.66877 & 2.83688 \\
\hline $\mathrm{H}$ & -0.40220 & 2.11123 & 3.20479 \\
\hline $\mathrm{H}$ & -1.70331 & 0.93183 & 3.03274 \\
\hline $\mathrm{H}$ & 1.37314 & -2.03815 & 3.16207 \\
\hline O & -2.34658 & 2.21972 & -0.78921 \\
\hline $\mathrm{H}$ & 2.28506 & -3.56857 & 3.18880 \\
\hline $\mathrm{H}$ & 1.33616 & 2.69418 & 1.91229 \\
\hline 0 & -4.11781 & -1.88464 & -1.90429 \\
\hline $\mathrm{H}$ & -4.34141 & -2.72317 & 0.57854 \\
\hline $\mathrm{H}$ & -3.80931 & -2.76487 & -2.17534 \\
\hline
\end{tabular}

Conformer 2

$\begin{array}{lrrr}\text { Energy: -1310.13662 } & \text { Hartree (Rel: } & 0.4 & \mathrm{kcal} / \mathrm{mol}) \\ \text { XYZ coordinates for } & \text { Conf } & 2 \text { : } & \\ \text { C } & 1.44809 & 3.74634 & -1.21006 \\ \text { C } & 1.83677 & 2.90338 & 0.01520 \\ \text { C } & 2.00577 & 1.38759 & -0.26224 \\ \text { C } & 0.64001 & 0.71863 & -0.49759 \\ \text { C } & -0.12668 & 0.53397 & 0.82051 \\ \text { C } & -1.15377 & 1.67995 & 1.07823 \\ \text { O } & -0.52943 & 2.94823 & 0.84195 \\ \text { C } & 0.84722 & 3.16429 & 1.17810 \\ \text { C } & 2.95694 & 1.01012 & -1.40827 \\ \text { C } & -2.30149 & 1.60814 & 0.02228 \\ \text { H } & 0.89938 & 4.22621 & 1.44438 \\ \text { C } & 3.20205 & -0.53168 & -1.48309 \\ \text { C } & 2.05553 & -1.36651 & -0.85401 \\ \text { C } & 0.69921 & -0.70409 & -1.10767 \\ \text { C } & -0.53981 & -1.39036 & -0.48430 \\ \text { O } & -0.70954 & -0.76842 & 0.80577 \\ \text { C } & -3.59158 & 0.83290 & 0.28738\end{array}$




\begin{tabular}{|c|c|c|c|}
\hline $\mathrm{C}$ & -3.55401 & -0.69925 & 0.49533 \\
\hline C & -3.16415 & -1.56813 & -0.72017 \\
\hline $\mathrm{H}$ & 2.16513 & 3.62369 & -2.02839 \\
\hline $\mathrm{H}$ & 1.43020 & 4.81025 & -0.94372 \\
\hline $\mathrm{H}$ & 0.45029 & 3.48660 & -1.57578 \\
\hline $\mathrm{H}$ & 2.80946 & 3.26979 & 0.37940 \\
\hline $\mathrm{H}$ & 2.43282 & 0.95791 & 0.65377 \\
\hline $\mathrm{H}$ & 0.05414 & 1.34998 & -1.16916 \\
\hline $\mathrm{H}$ & 0.56685 & 0.54083 & 1.66979 \\
\hline $\mathrm{C}$ & -1.67829 & 1.63433 & 2.51957 \\
\hline $\mathrm{H}$ & 2.53647 & 1.35722 & -2.36016 \\
\hline $\mathrm{H}$ & 3.91879 & 1.52538 & -1.29087 \\
\hline $\mathrm{H}$ & -2.91117 & -0.96043 & 1.33485 \\
\hline $\mathrm{H}$ & 3.19198 & -0.81391 & -2.54450 \\
\hline C & 4.57195 & -0.93442 & -0.91883 \\
\hline $\mathrm{H}$ & 2.07611 & -2.38095 & -1.25779 \\
\hline O & 2.27123 & -1.48102 & 0.58573 \\
\hline $\mathrm{H}$ & 0.58539 & -0.65501 & -2.19751 \\
\hline $\mathrm{H}$ & -0.36130 & -2.45075 & -0.28368 \\
\hline $\mathrm{H}$ & 4.71999 & -2.02020 & -0.96314 \\
\hline $\mathrm{H}$ & -4.05033 & 1.26880 & 1.18556 \\
\hline $\mathrm{H}$ & -4.24544 & 1.06249 & -0.55795 \\
\hline $\mathrm{H}$ & -4.57696 & -0.99396 & 0.76135 \\
\hline C & -3.23333 & -3.05076 & -0.32022 \\
\hline C & -1.79552 & -1.22572 & -1.36059 \\
\hline $\mathrm{H}$ & -3.02052 & -3.69371 & -1.18396 \\
\hline $\mathrm{H}$ & -2.51078 & -3.29563 & 0.46602 \\
\hline $\mathrm{H}$ & -1.85193 & -0.20582 & -1.75329 \\
\hline $\mathrm{H}$ & -1.66620 & -1.88098 & -2.23534 \\
\hline $\mathrm{H}$ & 5.37292 & -0.46178 & -1.49786 \\
\hline $\mathrm{H}$ & 4.68027 & -0.62375 & 0.12562 \\
\hline C & 2.09562 & -2.68549 & 1.17277 \\
\hline $\mathrm{C}$ & 2.28067 & -2.58330 & 2.66739 \\
\hline O & 1.80748 & -3.70437 & 0.57545 \\
\hline $\mathrm{H}$ & 3.16284 & -1.98608 & 2.91352 \\
\hline $\mathrm{H}$ & -2.39843 & 2.43985 & 2.69556 \\
\hline $\mathrm{H}$ & -0.84301 & 1.77029 & 3.21572 \\
\hline $\mathrm{H}$ & -2.15311 & 0.67656 & 2.75186 \\
\hline $\mathrm{H}$ & 1.40630 & -2.08058 & 3.09658 \\
\hline 0 & -2.17653 & 2.22037 & -1.02594 \\
\hline $\mathrm{H}$ & 2.36564 & -3.58222 & 3.09720 \\
\hline $\mathrm{H}$ & 1.13878 & 2.59576 & 2.07194 \\
\hline O & -4.18455 & -1.29559 & -1.70156 \\
\hline $\mathrm{H}$ & -4.23611 & -3.29481 & 0.04596 \\
\hline & -3.97505 & -1.81422 & -2.49565 \\
\hline
\end{tabular}

Conformer 3 XYZ coordinates for conf 3 :

$\begin{array}{lrrr}\text { C } & 1.42823 & 3.74062 & -1.23749 \\ \text { C } & 1.82634 & 2.90783 & -0.00839 \\ \text { C } & 2.00157 & 1.39113 & -0.27649 \\ \text { C } & 0.63828 & 0.71331 & -0.50169 \\ \text { C } & -0.11832 & 0.52560 & 0.82141 \\ \text { C } & -1.14474 & 1.67048 & 1.09381 \\ \text { O } & -0.53546 & 2.93949 & 0.82951 \\ \text { C } & 0.84134 & 3.17061 & 1.15741 \\ \text { C } & 2.95047 & 1.01025 & -1.42329 \\ \text { C } & -2.31623 & 1.58006 & 0.06871 \\ \text { H } & 0.88632 & 4.23461 & 1.41621 \\ \text { C } & 3.20370 & -0.53085 & -1.48709 \\ C & 2.06151 & -1.36707 & -0.85149 \\ C & 0.70290 & -0.71089 & -1.10819\end{array}$




\begin{tabular}{|c|c|c|c|}
\hline C & -0.53639 & -1.39731 & -0.48529 \\
\hline 0 & -0.70349 & -0.77551 & 0.80621 \\
\hline C & -3.59352 & 0.80134 & 0.37951 \\
\hline $\mathrm{C}$ & -3.54290 & -0.74036 & 0.51471 \\
\hline C & -3.16678 & -1.56115 & -0.74549 \\
\hline $\mathrm{H}$ & 2.14393 & 3.61783 & -2.05699 \\
\hline $\mathrm{H}$ & 1.40321 & 4.80602 & -0.97779 \\
\hline $\mathrm{H}$ & 0.43154 & 3.47151 & -1.59969 \\
\hline $\mathrm{H}$ & 2.79874 & 3.28165 & 0.34891 \\
\hline $\mathrm{H}$ & 2.43418 & 0.96954 & 0.64061 \\
\hline $\mathrm{H}$ & 0.04337 & 1.33930 & -1.17059 \\
\hline $\mathrm{H}$ & 0.58238 & 0.52991 & 1.66481 \\
\hline $\mathrm{C}$ & -1.63333 & 1.62957 & 2.54791 \\
\hline $\mathrm{H}$ & 2.52487 & 1.34794 & -2.37629 \\
\hline $\mathrm{H}$ & 3.90997 & 1.53146 & -1.31309 \\
\hline $\mathrm{H}$ & -2.88519 & -1.02335 & 1.33631 \\
\hline $\mathrm{H}$ & 3.19451 & -0.82055 & -2.54649 \\
\hline $\mathrm{C}$ & 4.57601 & -0.92213 & -0.92069 \\
\hline $\mathrm{H}$ & 2.08563 & -2.38367 & -1.24929 \\
\hline O & 2.27992 & -1.47176 & 0.58861 \\
\hline $\mathrm{H}$ & 0.59050 & -0.66519 & -2.19819 \\
\hline $\mathrm{H}$ & -0.36157 & -2.45841 & -0.28549 \\
\hline $\mathrm{H}$ & 4.72952 & -2.00742 & -0.95719 \\
\hline $\mathrm{H}$ & -4.00623 & 1.19323 & 1.31801 \\
\hline $\mathrm{H}$ & -4.29843 & 1.07733 & -0.41209 \\
\hline $\mathrm{H}$ & -4.55969 & -1.05108 & 0.78531 \\
\hline $\mathrm{C}$ & -3.27026 & -3.05286 & -0.41059 \\
\hline $\mathrm{C}$ & -1.78689 & -1.22773 & -1.36799 \\
\hline $\mathrm{H}$ & -3.05595 & -3.65785 & -1.29789 \\
\hline $\mathrm{H}$ & -2.56645 & -3.33114 & 0.38031 \\
\hline $\mathrm{H}$ & -1.81970 & -0.20153 & -1.75309 \\
\hline $\mathrm{H}$ & -1.66698 & -1.87513 & -2.24629 \\
\hline $\mathrm{H}$ & 5.37400 & -0.44961 & -1.50389 \\
\hline $\mathrm{H}$ & 4.68350 & -0.60332 & 0.12131 \\
\hline $\mathrm{C}$ & 2.10374 & -2.67227 & 1.18401 \\
\hline $\mathrm{C}$ & 2.29043 & -2.56016 & 2.67771 \\
\hline 0 & 1.81305 & -3.69437 & 0.59381 \\
\hline $\mathrm{H}$ & 3.16982 & -1.95715 & 2.91941 \\
\hline $\mathrm{H}$ & -2.36115 & 2.42526 & 2.73701 \\
\hline $\mathrm{H}$ & -0.78334 & 1.78249 & 3.22231 \\
\hline $\mathrm{H}$ & -2.08662 & 0.66556 & 2.79781 \\
\hline $\mathrm{H}$ & 1.41383 & -2.05968 & 3.10511 \\
\hline 0 & -2.22064 & 2.16876 & -0.99639 \\
\hline $\mathrm{H}$ & 2.38065 & -3.55616 & 3.11331 \\
\hline $\mathrm{H}$ & 1.14105 & 2.60992 & 2.05331 \\
\hline 0 & -4.16199 & -1.34587 & -1.76749 \\
\hline $\mathrm{H}$ & -4.28275 & -3.29407 & -0.06979 \\
\hline $\mathrm{H}$ & -4.04970 & -0.44227 & -2.10529 \\
\hline
\end{tabular}

Conformer 4

Energy: -1310.13507 Hartree (Rel: $1.4 \mathrm{kcal} / \mathrm{mol}$ ) XYZ coordinates for conf 4 :

$\begin{array}{lrrr}C & -0.38123 & 3.02683 & -2.43638 \\ C & 0.45188 & 2.97002 & -1.14540 \\ C & 1.17155 & 1.61945 & -0.88974 \\ C & 0.16793 & 0.52108 & -0.50007 \\ C & -0.24899 & 0.56408 & 0.97408 \\ C & -1.41234 & 1.54072 & 1.31313 \\ \text { O } & -1.54236 & 2.57610 & 0.32717 \\ C & -0.39211 & 3.40105 & 0.07239 \\ C & 2.03614 & 1.12246 & -2.06209 \\ C & -2.72443 & 0.70587 & 1.23831 \\ H & -0.79183 & 4.40556 & -0.10948\end{array}$




\begin{tabular}{|c|c|c|}
\hline 2.85536 & -0.15773 & -1.71549 \\
\hline 2.19359 & -1.02008 & -0.60703 \\
\hline 0.66944 & -0.92864 & -0.68368 \\
\hline-0.10440 & -1.72375 & 0.40148 \\
\hline-0.68281 & -0.76090 & 1.30153 \\
\hline-3.23146 & 0.20045 & -0.10660 \\
\hline-3.46682 & -1.33079 & -0.15778 \\
\hline-2.42209 & -2.22281 & -0.90416 \\
\hline 0.23398 & 2.84378 & -3.32299 \\
\hline-0.83424 & 4.01919 & -2.54829 \\
\hline-1.19594 & 2.29576 & -2.43192 \\
\hline 1.23557 & 3.73952 & -1.22976 \\
\hline 1.84591 & 1.78382 & -0.03684 \\
\hline-0.71630 & 0.62416 & -1.12659 \\
\hline 0.62488 & 0.81362 & 1.59174 \\
\hline-1.26090 & 2.11899 & 2.72236 \\
\hline 1.38623 & 0.90967 & -2.91944 \\
\hline 2.72243 & 1.91545 & -2.38681 \\
\hline-3.61349 & -1.71166 & 0.85860 \\
\hline 2.84566 & -0.80111 & -2.60557 \\
\hline 4.32480 & 0.15610 & -1.39904 \\
\hline 2.53295 & -2.05345 & -0.69995 \\
\hline 2.61618 & -0.56002 & 0.71443 \\
\hline 0.38768 & -1.28984 & -1.67570 \\
\hline 0.60426 & -2.33546 & 0.97874 \\
\hline 4.87747 & -0.75011 & -1.12567 \\
\hline-4.20227 & 0.69644 & -0.23658 \\
\hline-2.60720 & 0.52806 & -0.93555 \\
\hline-4.42248 & -1.48826 & -0.67337 \\
\hline-3.15865 & -3.50013 & -1.35923 \\
\hline-1.19713 & -2.69068 & -0.07735 \\
\hline-2.47844 & -4.16646 & -1.89968 \\
\hline-3.99010 & -3.24305 & -2.02747 \\
\hline-0.68565 & -3.46474 & -0.66515 \\
\hline-1.59149 & -3.19564 & 0.81322 \\
\hline 4.81349 & 0.59898 & -2.27388 \\
\hline 4.41188 & 0.86714 & -0.57019 \\
\hline 3.39071 & -1.37179 & 1.47062 \\
\hline 3.70716 & -0.72075 & 2.79621 \\
\hline 3.77115 & -2.47316 & 1.12643 \\
\hline 4.20665 & 0.24001 & 2.63511 \\
\hline-2.10792 & 2.76624 & 2.96526 \\
\hline-0.33616 & 2.69709 & 2.81303 \\
\hline-1.22730 & 1.30875 & 3.45545 \\
\hline 2.78075 & -0.52187 & 3.34510 \\
\hline-3.35972 & 0.47258 & 2.25080 \\
\hline 4.34929 & -1.37803 & 3.38347 \\
\hline 0.24440 & 3.46107 & 0.96344 \\
\hline-1.91132 & -1.55182 & -2.07095 \\
\hline-3.57624 & -4.04685 & -0.50597 \\
\hline-2.66353 & -1.36974 & -2.65870 \\
\hline
\end{tabular}


Asbestinin-21 - first revision (the 7-epimer of the final revision below)

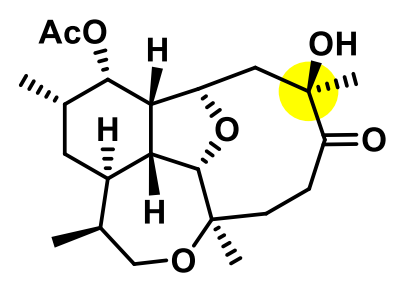

Rel energy (kcal/mol): $\begin{array}{ccc}\text { Conf } 1 & \operatorname{Conf} 2 \\ 0.0 & 0.2\end{array}$

\begin{tabular}{|c|c|c|c|c|c|c|c|}
\hline C-nom & iGau & $\operatorname{Exp}$ & Calc & $\operatorname{diff}$ & 1 & 2 & \\
\hline $\mathrm{C} 1-\mathrm{CH}$ & 4 & 38.03 & 39.06 & 1.03 & 39.15 & $38.94]$ & \\
\hline $\mathrm{C} 2-\mathrm{CH}$ & 5 & 93.64 & 94.03 & 0.39 & 94.37 & $93.55]$ & \\
\hline C3-C & 6 & 77.21 & 77.72 & 0.51 & 77.66 & 77.80 ] & \\
\hline $\mathrm{C} 4-\mathrm{C} \star \star$ & 10 & 34.03 & 34.34 & 0.31 & 33.53 & $35.46]$ & \\
\hline $\mathrm{C} 5-\mathrm{CH} 2$ & 17 & 36.19 & 34.38 & -1.81 & 32.95 & $36.35]$ & \\
\hline $\mathrm{C} 6-\mathrm{CH} 2 * *$ & 18 & 210.78 & 212.31 & 1.53 & [ 214.54 & $209.23]$ & \\
\hline $\mathrm{C} 7-\mathrm{C}$ & 19 & 76.29 & 78.63 & 2.34 & {$[78.76$} & $78.44]$ & \\
\hline $\mathrm{C} 8-\mathrm{CH} 2$ & 41 & 49.33 & 48.02 & -1.31 & 48.46 & $47.40]$ & \\
\hline $\mathrm{C} 9-\mathrm{CH}$ & 15 & 78.23 & 78.79 & 0.56 & 78.45 & $79.26]$ & \\
\hline $\mathrm{ClO}-\mathrm{CH}$ & 14 & 47.73 & 49.22 & 1.49 & 49.48 & $48.86]$ & \\
\hline $\mathrm{C} 11-\mathrm{CH}$ & 13 & 72.79 & 73.13 & 0.34 & 73.17 & $73.07]$ & \\
\hline $\mathrm{C} 12-\mathrm{CH}$ & 12 & 31.51 & 32.49 & 0.98 & 32.55 & $32.41]$ & \\
\hline $\mathrm{C} 13-\mathrm{CH} 2$ & 9 & 31.51 & 30.83 & -0.68 & 30.88 & $30.77]$ & \\
\hline $\mathrm{Cl} 4-\mathrm{CH}$ & 3 & 38.03 & 38.66 & 0.63 & 38.60 & $38.74]$ & \\
\hline $\mathrm{C} 15-\mathrm{CH}$ & 2 & 36.52 & 36.91 & 0.39 & 36.79 & $37.08]$ & \\
\hline $\mathrm{C} 16-\mathrm{CH} 2$ & 8 & 68.17 & 68.12 & -0.05 & 68.37 & $67.78]$ & \\
\hline $\mathrm{C} 17-\mathrm{CH} 3$ & 1 & 10.91 & 11.39 & 0.48 & 11.44 & $11.33]$ & \\
\hline $\mathrm{C} 18-\mathrm{CH} 3$ & 27 & 22.50 & 21.70 & -0.80 & 21.65 & 21.77 ] & \\
\hline $\mathrm{C} 19-\mathrm{CH} 3$ & 40 & 27.66 & 22.78 & -4.88 & 21.97 & $23.91]$ & \\
\hline $\mathrm{C} 20-\mathrm{CH} 3$ & 32 & 17.25 & 17.05 & -0.20 & [ 16.97 & $17.16]$ & \\
\hline $\mathrm{C} 21-\mathrm{C}$ & 48 & 170.84 & 171.53 & 0.69 & [ 171.50 & 171.57 ] & \\
\hline $\mathrm{C} 22-\mathrm{CH} 3$ & 49 & 21.34 & 20.81 & -0.53 & [ 20.88 & $20.72]$ & \\
\hline
\end{tabular}

Conformer 1

Energy: -1310.14122 Hartree (Rel: $0.0 \mathrm{kcal} / \mathrm{mol}$ )

XYZ coordinates for conf 1 :

$\begin{array}{lrrr}\text { C } & 1.72470 & 3.60310 & -1.57015 \\ \text { C } & 2.10050 & 2.83768 & -0.29162 \\ \text { C } & 2.14101 & 1.29621 & -0.44170 \\ \text { C } & 0.72373 & 0.70135 & -0.55187 \\ \text { C } & 0.02249 & 0.61724 & 0.81242 \\ \text { C } & -0.84653 & 1.86363 & 1.14972 \\ \text { O } & -0.20424 & 3.05597 & 0.64326 \\ \text { C } & 1.19322 & 3.25955 & 0.88615 \\ \text { C } & 3.00929 & 0.76348 & -1.59263 \\ \text { C } & -2.19303 & 1.85188 & 0.39576 \\ \text { H } & 1.30675 & 4.33794 & 1.05061 \\ \text { C } & 3.16950 & -0.78837 & -1.54233 \\ \text { C } & 1.98916 & -1.49572 & -0.82584 \\ \text { C } & 0.67873 & -0.75128 & -1.08830 \\ \text { C } & -0.59421 & -1.31855 & -0.41993 \\ \text { O } & -0.75225 & -0.58928 & 0.81156 \\ \text { C } & -3.24174 & 0.79819 & 0.87789 \\ \text { C } & -3.82879 & 0.02454 & -0.28385\end{array}$




\begin{tabular}{|c|c|c|c|}
\hline $\mathrm{C}$ & -3.24941 & -1.33670 & -0.76675 \\
\hline $\mathrm{H}$ & 2.41710 & 3.38418 & -2.38949 \\
\hline $\mathrm{H}$ & 1.75980 & 4.68390 & -1.38756 \\
\hline $\mathrm{H}$ & 0.71081 & 3.36354 & -1.90553 \\
\hline $\mathrm{H}$ & 3.11468 & 3.15589 & -0.00336 \\
\hline $\mathrm{H}$ & 2.58368 & 0.91450 & 0.48801 \\
\hline $\mathrm{H}$ & 0.12987 & 1.32631 & -1.22614 \\
\hline $\mathrm{H}$ & 0.77816 & 0.50260 & 1.60061 \\
\hline $\mathrm{C}$ & -1.05223 & 1.99830 & 2.66506 \\
\hline $\mathrm{H}$ & 2.55996 & 1.05202 & -2.55102 \\
\hline $\mathrm{H}$ & 4.00140 & 1.23217 & -1.56611 \\
\hline $\mathrm{H}$ & -1.98406 & 1.73356 & -0.67243 \\
\hline $\mathrm{H}$ & 3.12130 & -1.15601 & -2.57606 \\
\hline C & 4.52708 & -1.21947 & -0.96970 \\
\hline $\mathrm{H}$ & 1.92169 & -2.53222 & -1.16221 \\
\hline O & 2.24017 & -1.53485 & 0.61170 \\
\hline $\mathrm{H}$ & 0.55308 & -0.74866 & -2.17794 \\
\hline $\mathrm{H}$ & -0.47169 & -2.36885 & -0.13775 \\
\hline $\mathrm{H}$ & 4.61235 & -2.31171 & -0.92106 \\
\hline $\mathrm{H}$ & -2.79393 & 0.11209 & 1.59268 \\
\hline $\mathrm{H}$ & -4.07881 & 1.31051 & 1.36137 \\
\hline C & -3.33744 & -2.38883 & 0.35348 \\
\hline $\mathrm{C}$ & -1.81240 & -1.18478 & -1.35264 \\
\hline $\mathrm{H}$ & -3.02496 & -3.35866 & -0.04833 \\
\hline $\mathrm{H}$ & -2.70122 & -2.14547 & 1.20754 \\
\hline $\mathrm{H}$ & -1.76314 & -0.24312 & -1.91471 \\
\hline $\mathrm{H}$ & -1.72763 & -1.98158 & -2.10128 \\
\hline $\mathrm{H}$ & 5.33919 & -0.84653 & -1.60349 \\
\hline $\mathrm{H}$ & 4.67843 & -0.82600 & 0.04089 \\
\hline C & 2.03807 & -2.69721 & 1.27290 \\
\hline $\mathrm{C}$ & 2.29446 & -2.52231 & 2.75030 \\
\hline 0 & 1.68017 & -3.73210 & 0.74581 \\
\hline $\mathrm{H}$ & 3.22194 & -1.96945 & 2.92290 \\
\hline $\mathrm{H}$ & -1.70101 & 2.85191 & 2.88833 \\
\hline $\mathrm{H}$ & -0.09494 & 2.15324 & 3.17432 \\
\hline $\mathrm{H}$ & -1.50279 & 1.09562 & 3.09019 \\
\hline $\mathrm{H}$ & 1.47407 & -1.94203 & 3.18807 \\
\hline O & -4.80191 & 0.46526 & -0.88591 \\
\hline $\mathrm{H}$ & 2.33906 & -3.49902 & 3.23371 \\
\hline $\mathrm{H}$ & 1.51238 & 2.75728 & 1.80890 \\
\hline O & -4.07261 & -1.76693 & -1.84547 \\
\hline $\mathrm{H}$ & -4.37446 & -2.48560 & 0.69227 \\
\hline $\mathrm{H}$ & -4.74735 & -1.06206 & -1.94027 \\
\hline $\mathrm{H}$ & -2.62128 & 2.85351 & 0.49843 \\
\hline
\end{tabular}

Conformer 2

$\begin{array}{lrrr}\text { Energy: -1310.14091 Hartree (Rel: } & 0.2 \mathrm{kcal} / \mathrm{mol}) \\ \text { XYZ coordinates for } & \text { conf } 2: & & \\ \text { C } & 2.16798 & 3.57160 & -1.28923 \\ \text { C } & 2.29815 & 2.72000 & -0.01663 \\ \text { C } & 2.24148 & 1.18825 & -0.24878 \\ \text { C } & 0.80862 & 0.72069 & -0.57113 \\ \text { C } & -0.07439 & 0.68212 & 0.68585 \\ \text { C } & -0.91591 & 1.96844 & 0.89134 \\ \text { O } & -0.08996 & 3.12196 & 0.59034 \\ \text { C } & 1.26670 & 3.17027 & 1.04569 \\ \text { C } & 3.20810 & 0.63930 & -1.30976 \\ \text { C } & -2.06581 & 2.10987 & -0.13224 \\ \text { H } & 1.45278 & 4.22358 & 1.28844 \\ \text { C } & 3.23401 & -0.92165 & -1.33274 \\ \text { C } & 1.93220 & -1.55937 & -0.78315 \\ \text { C } & 0.71239 & -0.71220 & -1.15305 \\ \text { C } & -0.65000 & -1.21063 & -0.62267\end{array}$




$\begin{array}{rrr}-0.89612 & -0.48867 & 0.59981 \\ -3.39132 & 1.35121 & 0.10662 \\ -3.42448 & -0.17220 & 0.26332 \\ -3.20144 & -1.09031 & -0.98183 \\ 2.94708 & 3.32811 & -2.01871 \\ 2.26568 & 4.63587 & -1.04322 \\ 1.19355 & 3.43826 & -1.76981 \\ 3.28317 & 2.93678 & 0.42558 \\ 2.52713 & 0.72758 & 0.70575 \\ 0.37599 & 1.41015 & -1.30185 \\ 0.55814 & 0.54304 & 1.57140 \\ -1.42621 & 2.05699 & 2.33422 \\ 2.91426 & 1.01308 & -2.29858 \\ 4.22278 & 1.01629 & -1.12814 \\ -1.67953 & 1.90526 & -1.13469 \\ 3.26804 & -1.23472 & -2.38493 \\ 4.48165 & -1.49553 & -0.64631 \\ 1.83028 & -2.57614 & -1.16756 \\ 2.01104 & -1.66450 & 0.67062 \\ 0.69132 & -0.67694 & -2.24931 \\ -0.61664 & -2.27189 & -0.35626 \\ 4.47127 & -2.59210 & -0.64793 \\ -3.87370 & 1.73905 & 1.00859 \\ -4.04477 & 1.59171 & -0.74120 \\ -3.52243 & -2.54430 & -0.62772 \\ -1.79984 & -0.95425 & -1.60393 \\ -3.41817 & -3.16923 & -1.52055 \\ -2.85766 & -2.92383 & 0.15290 \\ -1.71054 & 0.04737 & -2.03650 \\ -1.73180 & -1.66069 & -2.44098 \\ 5.38594 & -1.16661 & -1.17003 \\ 4.55282 & -1.16187 & 0.39421 \\ 1.69083 & -2.84215 & 1.25452 \\ 1.77194 & -2.73183 & 2.75762 \\ 1.36299 & -3.84152 & 0.64552 \\ 2.68565 & -2.21452 & 3.06279 \\ -2.04012 & 2.95397 & 2.47084 \\ -0.58741 & 2.10757 & 3.03772 \\ -2.02738 & 1.17836 & 2.58941 \\ 0.91946 & -2.14423 & 3.11735 \\ -3.82669 & -0.65883 & 1.30851 \\ -1.73232 & -3.72717 & 3.20187 \\ -1.39796 & 2.59889 & 1.97428 \\ -2.32232 & -0.62237 & -0.01322 \\ -09542 & -2.63425 & .13365 \\ -1.175090 & & \end{array}$


Asbestinin-21 - second and final revision

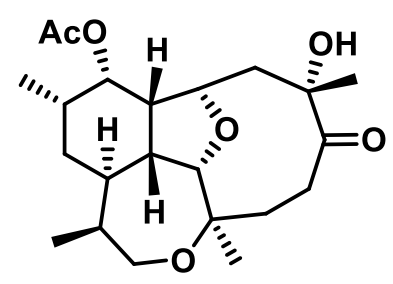

Conf 1 Conf 2

Rel energy (kcal/mol): $\begin{array}{cc}1.7 & 0.0\end{array}$

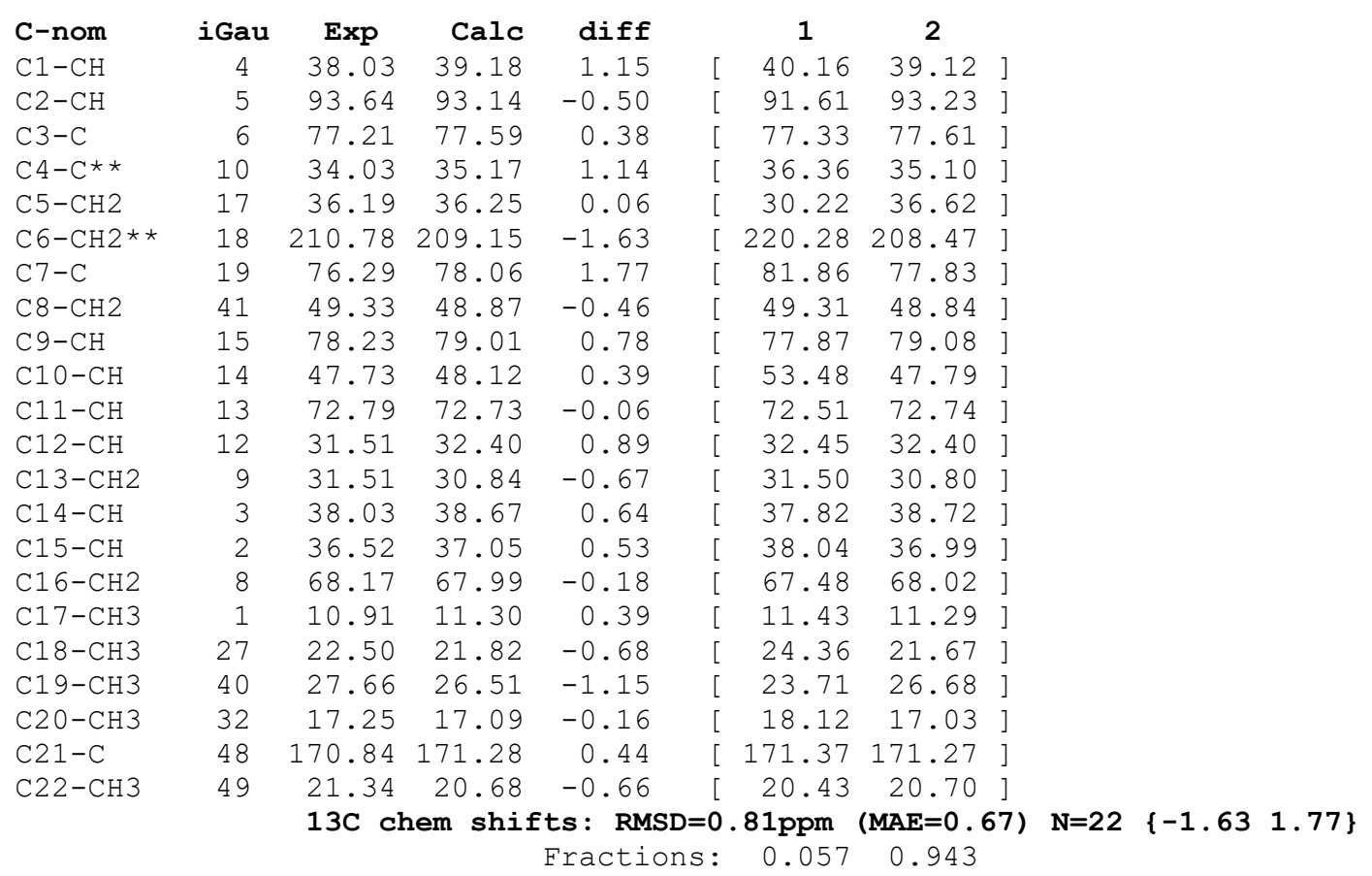

Conformer 1

Energy: -1310.14639 Hartree (Rel: $1.7 \mathrm{kcal} / \mathrm{mol}$ )

XYZ coordinates for conf 1 :

$\begin{array}{rrrr}\text { C } & 0.84237 & 3.83541 & -1.43861 \\ \text { C } & 1.33657 & 3.10289 & -0.18072 \\ \text { C } & 1.60800 & 1.58683 & -0.36756 \\ \text { C } & 0.32176 & 0.74794 & -0.48694 \\ \text { C } & -0.46959 & 0.69129 & 0.82610 \\ \text { C } & -1.53636 & 1.79776 & 1.03293 \\ \text { O } & -0.97582 & 3.09311 & 0.72722 \\ \text { C } & 0.39349 & 3.38380 & 1.00940 \\ \text { C } & 2.54640 & 1.26569 & -1.54544 \\ \text { C } & -2.73635 & 1.73821 & 0.05109 \\ \text { H } & 0.41527 & 4.45843 & 1.22638 \\ \text { C } & 2.93879 & -0.24276 & -1.63327 \\ \text { C } & 2.05679 & -1.14902 & -0.74683 \\ \text { C } & 0.57927 & -0.75083 & -0.83691 \\ \text { C } & -0.35478 & -1.56448 & 0.10105 \\ \text { O } & -1.10753 & -0.58925 & 0.85797 \\ \text { C } & -3.59911 & 0.46199 & -0.00683 \\ \text { C } & -2.96807 & -0.61367 & -0.88636 \\ \text { C } & -2.84489 & -2.07821 & -0.39467 \\ \text { H } & 1.57028 & 3.77208 & -2.25340\end{array}$




\begin{tabular}{|c|c|c|c|}
\hline $\mathrm{H}$ & 0.68416 & 4.89824 & -1.21918 \\
\hline $\mathrm{H}$ & -0.10978 & 3.43251 & -1.79723 \\
\hline $\mathrm{H}$ & 2.30111 & 3.55271 & 0.10306 \\
\hline $\mathrm{H}$ & 2.11896 & 1.26174 & 0.54752 \\
\hline $\mathrm{H}$ & -0.31371 & 1.16749 & -1.27216 \\
\hline $\mathrm{H}$ & 0.23708 & 0.72960 & 1.66833 \\
\hline C & -2.00885 & 1.79060 & 2.49428 \\
\hline $\mathrm{H}$ & 2.06353 & 1.56610 & -2.48286 \\
\hline $\mathrm{H}$ & 3.45583 & 1.87453 & -1.46145 \\
\hline $\mathrm{H}$ & -2.36875 & 1.94379 & -0.95854 \\
\hline $\mathrm{H}$ & 2.73032 & -0.58468 & -2.65610 \\
\hline C & 4.43446 & -0.47044 & -1.37269 \\
\hline $\mathrm{H}$ & 2.18983 & -2.19027 & -1.04825 \\
\hline O & 2.48487 & -1.05961 & 0.64755 \\
\hline $\mathrm{H}$ & 0.28862 & -0.92398 & -1.87774 \\
\hline $\mathrm{H}$ & 0.25170 & -2.13459 & 0.81345 \\
\hline $\mathrm{H}$ & 4.69330 & -1.53463 & -1.41642 \\
\hline $\mathrm{H}$ & -3.84837 & 0.07164 & 0.98001 \\
\hline $\mathrm{H}$ & -4.54967 & 0.72517 & -0.49186 \\
\hline C & -3.78769 & -2.96080 & -1.22190 \\
\hline $\mathrm{C}$ & -1.36979 & -2.51923 & -0.58703 \\
\hline $\mathrm{H}$ & -3.64630 & -4.01213 & -0.95054 \\
\hline $\mathrm{H}$ & -3.59214 & -2.83586 & -2.28984 \\
\hline $\mathrm{H}$ & -1.15514 & -2.58255 & -1.65725 \\
\hline $\mathrm{H}$ & -1.28018 & -3.52922 & -0.17394 \\
\hline $\mathrm{H}$ & 5.03454 & 0.05591 & -2.12306 \\
\hline $\mathrm{H}$ & 4.72595 & -0.09032 & -0.38709 \\
\hline C & 3.05726 & -2.14402 & 1.22409 \\
\hline $\mathrm{C}$ & 3.46427 & -1.83565 & 2.64527 \\
\hline O & 3.21808 & -3.21095 & 0.66718 \\
\hline $\mathrm{H}$ & 4.23998 & -1.06231 & 2.64899 \\
\hline $\mathrm{H}$ & -2.75379 & 2.57647 & 2.65507 \\
\hline $\mathrm{H}$ & -1.16697 & 1.97285 & 3.17227 \\
\hline $\mathrm{H}$ & -2.45215 & 0.82728 & 2.76798 \\
\hline $\mathrm{H}$ & 2.61089 & -1.44697 & 3.20949 \\
\hline O & -2.57119 & -0.33820 & -2.01104 \\
\hline $\mathrm{H}$ & 3.84679 & -2.73901 & 3.12140 \\
\hline $\mathrm{H}$ & 0.73962 & 2.86714 & 1.91525 \\
\hline O & -3.24345 & -2.23538 & 0.96510 \\
\hline $\mathrm{H}$ & -4.83023 & -2.69352 & -1.01865 \\
\hline $\mathrm{H}$ & -2.61831 & -1.69728 & 1.48303 \\
\hline $\mathrm{H}$ & -3.37557 & 2.58615 & 0.32091 \\
\hline
\end{tabular}

Conformer 2

$\begin{array}{lrrr}\text { Energy: -1310.14904 Hartree (Rel: } & 0.0 \mathrm{kcal} / \mathrm{mol}) \\ \text { XYZ coordinates for } & \text { conf } 2 \text { : } & \\ \text { C } & 2.12574 & 3.58809 & -1.31132 \\ \text { C } & 2.27329 & 2.74244 & -0.03670 \\ \text { C } & 2.22759 & 1.20964 & -0.26154 \\ \text { C } & 0.79786 & 0.72876 & -0.57769 \\ \text { C } & -0.07885 & 0.67519 & 0.68258 \\ \text { C } & -0.91856 & 1.95790 & 0.91419 \\ \text { O } & -0.11414 & 3.11884 & 0.59215 \\ \text { C } & 1.24728 & 3.18573 & 1.03287 \\ \text { C } & 3.19685 & 0.66214 & -1.32080 \\ \text { C } & -2.09946 & 2.08965 & -0.07536 \\ \text { H } & 1.42275 & 4.24188 & 1.27077 \\ \text { C } & 3.23740 & -0.89847 & -1.33160 \\ \text { C } & 1.93657 & -1.54413 & -0.78758 \\ \text { C } & 0.71387 & -0.70402 & -1.16200 \\ \text { C } & -0.64938 & -1.20825 & -0.64472 \\ \text { O } & -0.91022 & -0.48674 & 0.57795 \\ \text { C } & -3.40049 & 1.30622 & 0.20676\end{array}$




\begin{tabular}{|c|c|c|c|}
\hline $\mathrm{C}$ & -3.41602 & -0.21796 & 0.26039 \\
\hline C & -3.18263 & -1.11484 & -0.99161 \\
\hline $\mathrm{H}$ & 2.89992 & 3.34692 & -2.04678 \\
\hline $\mathrm{H}$ & 2.21840 & 4.65397 & -1.07041 \\
\hline $\mathrm{H}$ & 1.14804 & 3.44613 & -1.78288 \\
\hline $\mathrm{H}$ & 3.25968 & 2.96979 & 0.39697 \\
\hline $\mathrm{H}$ & 2.51895 & 0.75588 & 0.69476 \\
\hline $\mathrm{H}$ & 0.35224 & 1.41533 & -1.30385 \\
\hline $\mathrm{H}$ & 0.55837 & 0.51800 & 1.56194 \\
\hline C & -1.38897 & 2.03841 & 2.37174 \\
\hline $\mathrm{H}$ & 2.89736 & 1.02579 & -2.31176 \\
\hline $\mathrm{H}$ & 4.20830 & 1.05006 & -1.14420 \\
\hline $\mathrm{H}$ & -1.74173 & 1.89808 & -1.09113 \\
\hline $\mathrm{H}$ & 3.28345 & -1.21860 & -2.38118 \\
\hline C & 4.48468 & -1.45565 & -0.63087 \\
\hline $\mathrm{H}$ & 1.84236 & -2.56105 & -1.17311 \\
\hline O & 2.00430 & -1.64986 & 0.66622 \\
\hline $\mathrm{H}$ & 0.70059 & -0.66596 & -2.25842 \\
\hline $\mathrm{H}$ & -0.63649 & -2.26962 & -0.38256 \\
\hline $\mathrm{H}$ & 4.48413 & -2.55220 & -0.62485 \\
\hline $\mathrm{H}$ & -3.82311 & 1.62955 & 1.16223 \\
\hline $\mathrm{H}$ & -4.12157 & 1.60441 & -0.56685 \\
\hline C & -4.29194 & -0.80772 & -2.02188 \\
\hline C & -1.79319 & -0.94837 & -1.63222 \\
\hline $\mathrm{H}$ & -4.22246 & -1.53426 & -2.83767 \\
\hline $\mathrm{H}$ & -4.20100 & 0.19759 & -2.44663 \\
\hline $\mathrm{H}$ & -1.70052 & 0.05126 & -2.07074 \\
\hline $\mathrm{H}$ & -1.72165 & -1.66441 & -2.46099 \\
\hline $\mathrm{H}$ & 5.39029 & -1.12217 & -1.14944 \\
\hline $\mathrm{H}$ & 4.54439 & -1.11433 & 0.40793 \\
\hline C & 1.72615 & -2.84269 & 1.24269 \\
\hline C & 1.77067 & -2.72966 & 2.74717 \\
\hline O & 1.45923 & -3.85509 & 0.62654 \\
\hline $\mathrm{H}$ & 2.66410 & -2.18861 & 3.07117 \\
\hline $\mathrm{H}$ & -2.02404 & 2.91755 & 2.52613 \\
\hline $\mathrm{H}$ & -0.53101 & 2.11537 & 3.04884 \\
\hline $\mathrm{H}$ & -1.95393 & 1.14296 & 2.65103 \\
\hline $\mathrm{H}$ & 0.89609 & -2.16372 & 3.08781 \\
\hline 0 & -3.82667 & -0.77865 & 1.27066 \\
\hline $\mathrm{H}$ & 1.74829 & -3.72544 & 3.19152 \\
\hline $\mathrm{H}$ & 1.39440 & 2.61822 & 1.96138 \\
\hline O & -3.31099 & -2.46525 & -0.57125 \\
\hline $\mathrm{H}$ & -5.28044 & -0.90860 & -1.56076 \\
\hline $\mathrm{H}$ & -3.62189 & -2.40064 & 0.35719 \\
\hline $\mathrm{H}$ & -2.37503 & 3.15034 & -0.05866 \\
\hline
\end{tabular}

